\title{
WestVirginiaUniversity
}

THE RESEARCH REPOSITORY @ WVU

Graduate Theses, Dissertations, and Problem Reports

2014

\section{Concerto for Solo Violin and Chamber Orchestra}

Christopher Everett Jones

West Virginia University

Follow this and additional works at: https://researchrepository.wvu.edu/etd

\section{Recommended Citation}

Jones, Christopher Everett, "Concerto for Solo Violin and Chamber Orchestra" (2014). Graduate Theses, Dissertations, and Problem Reports. 381.

https://researchrepository.wvu.edu/etd/381

This Thesis is protected by copyright and/or related rights. It has been brought to you by the The Research Repository @ WVU with permission from the rights-holder(s). You are free to use this Thesis in any way that is permitted by the copyright and related rights legislation that applies to your use. For other uses you must obtain permission from the rights-holder(s) directly, unless additional rights are indicated by a Creative Commons license in the record and/ or on the work itself. This Thesis has been accepted for inclusion in WVU Graduate Theses, Dissertations, and Problem Reports collection by an authorized administrator of The Research Repository @ WVU. For more information, please contact researchrepository@mail.wvu.edu. 


\title{
Concerto for Solo Violin and Chamber Orchestra
}

\author{
Christopher Everett Jones \\ Thesis submitted \\ to the College of Creative Arts \\ at West Virginia University \\ in partial fulfillment of the requirements for the degree of \\ Master of Music in \\ Composition \\ John Beall, Ph.D., Chair \\ John Crotty, Ph.D. \\ C.B. Wilson, Ph.D. \\ School of Music \\ Morgantown, West Virginia \\ 2014
}

Keywords: Music, Score, Concerto, Violin, Orchestra, Chamber Orchestra

Copyright 2013 Christopher Everett Jones 


\section{Abstract \\ Concerto for Solo Violin and Chamber Orchestra \\ Christopher Everett Jones}

The Concerto for Violin and Chamber Orchestra was composed over the period of approximately one week in September 2013. It was previewed two weeks later with soloist Taylor Giorgio and Conductor Eleanor Aban in a masterclass with guest-composer and WVU alumnus Jay Chattaway (of Star Trek fame).

The piece is continuous but formally divided into three parts. The first section is in a slow to moderate tempo and fluctuates between B minor and B major (with significant chromaticism) and harmonies that never fully resolve until the end of the piece. The second section begins in a brisk compound meter, reminiscent of a dance, and is suggestive of $\mathrm{D}$ major. The development of this section culminates in a driving rhythm as well as the dissolution of any tonality that had been present before. A cadenza for the solo violin contemplatively reflects on the ideas from earlier in the piece and the orchestra joins on a long, sustained unison F\#. As the tempo from the beginning returns, the third and final section of the piece begins. The concept of $\mathrm{B}$ minor versus $\mathrm{B}$ major from the beginning returns and ultimately resolves to $\mathrm{B}$ major. 


\section{Score}

\section{For Violin Solo and Chamber Orchestra}

C. E. Jones

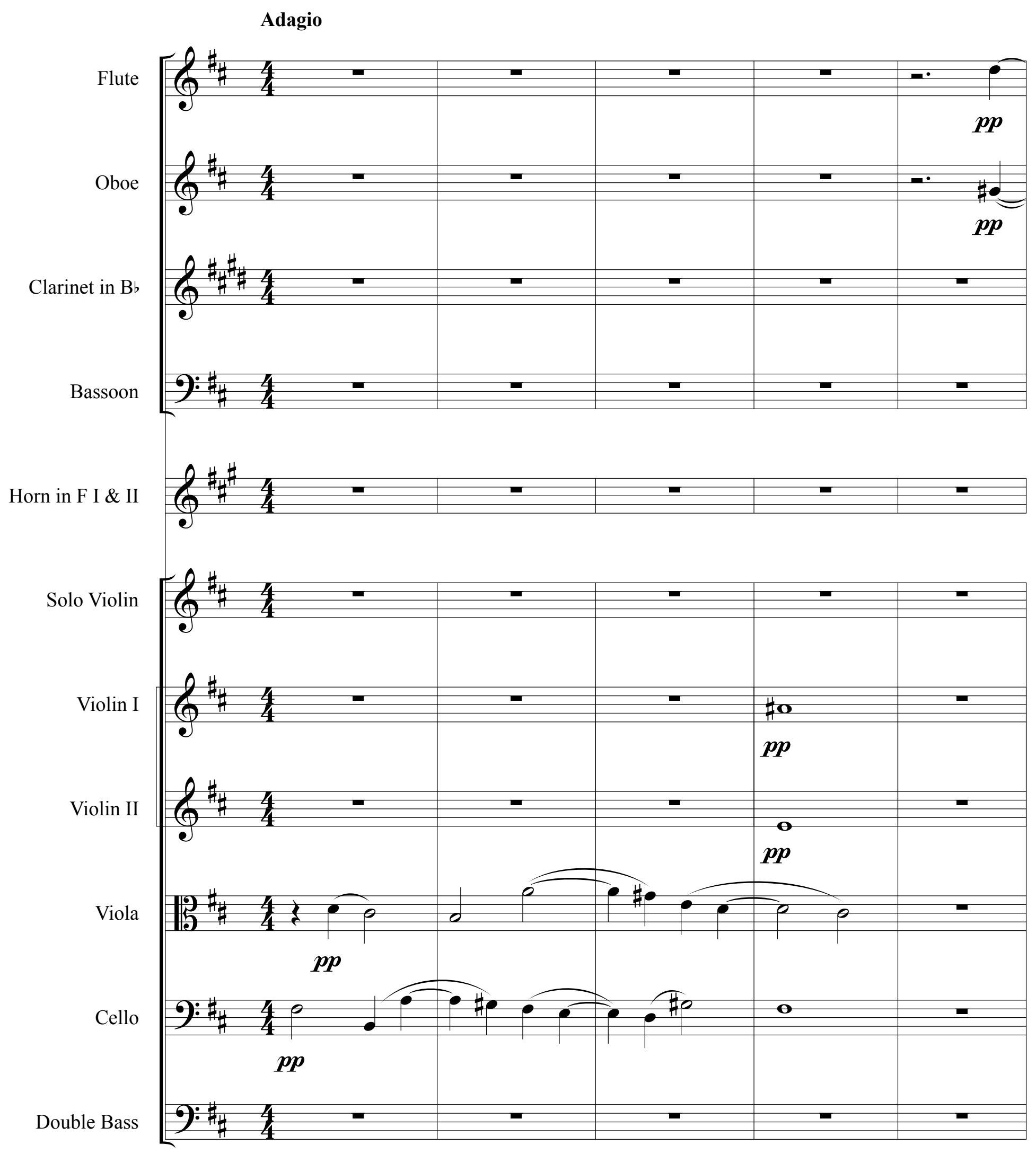


4

For Violin Solo and Chamber Orchestra

Fl.
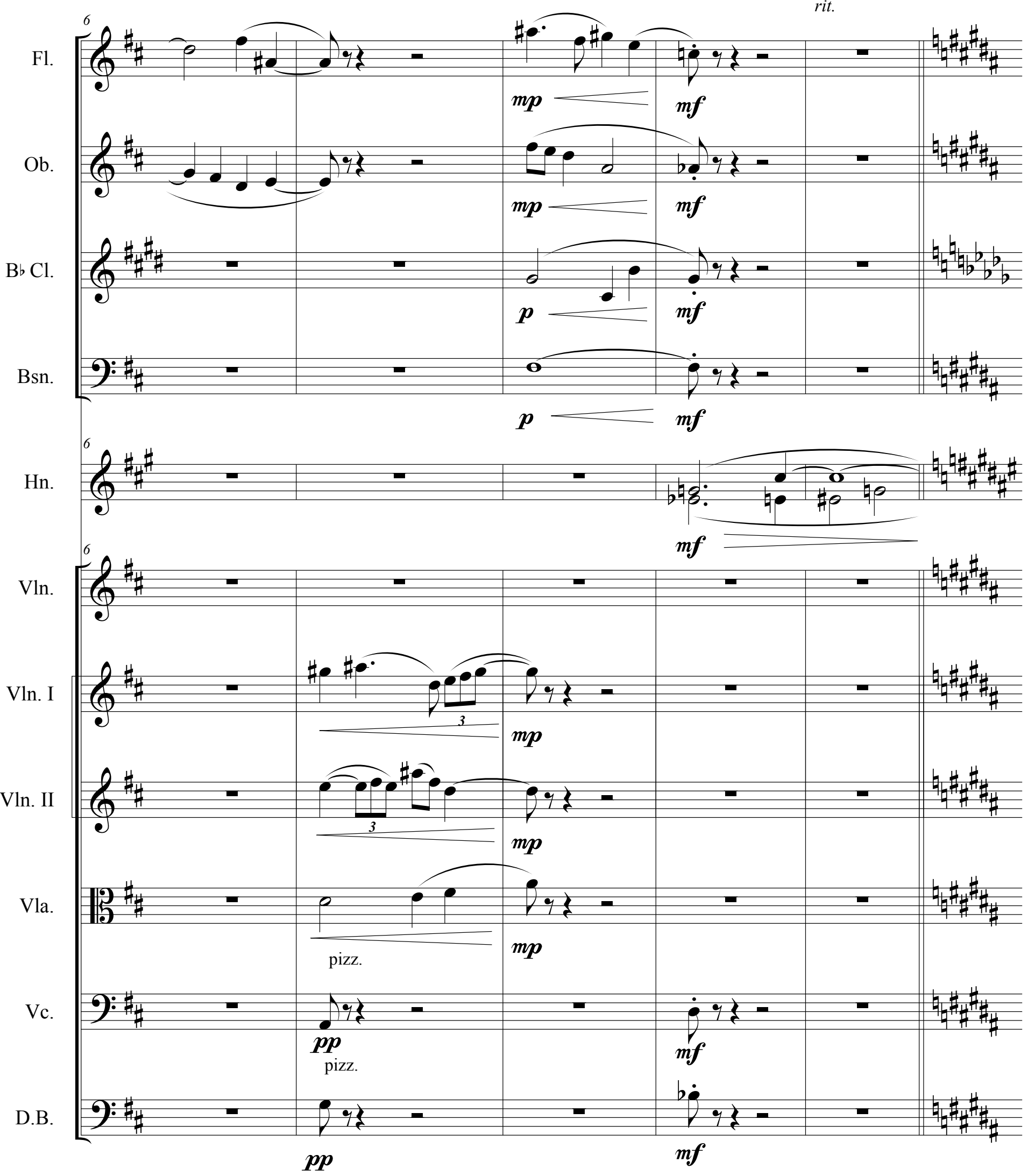
Score

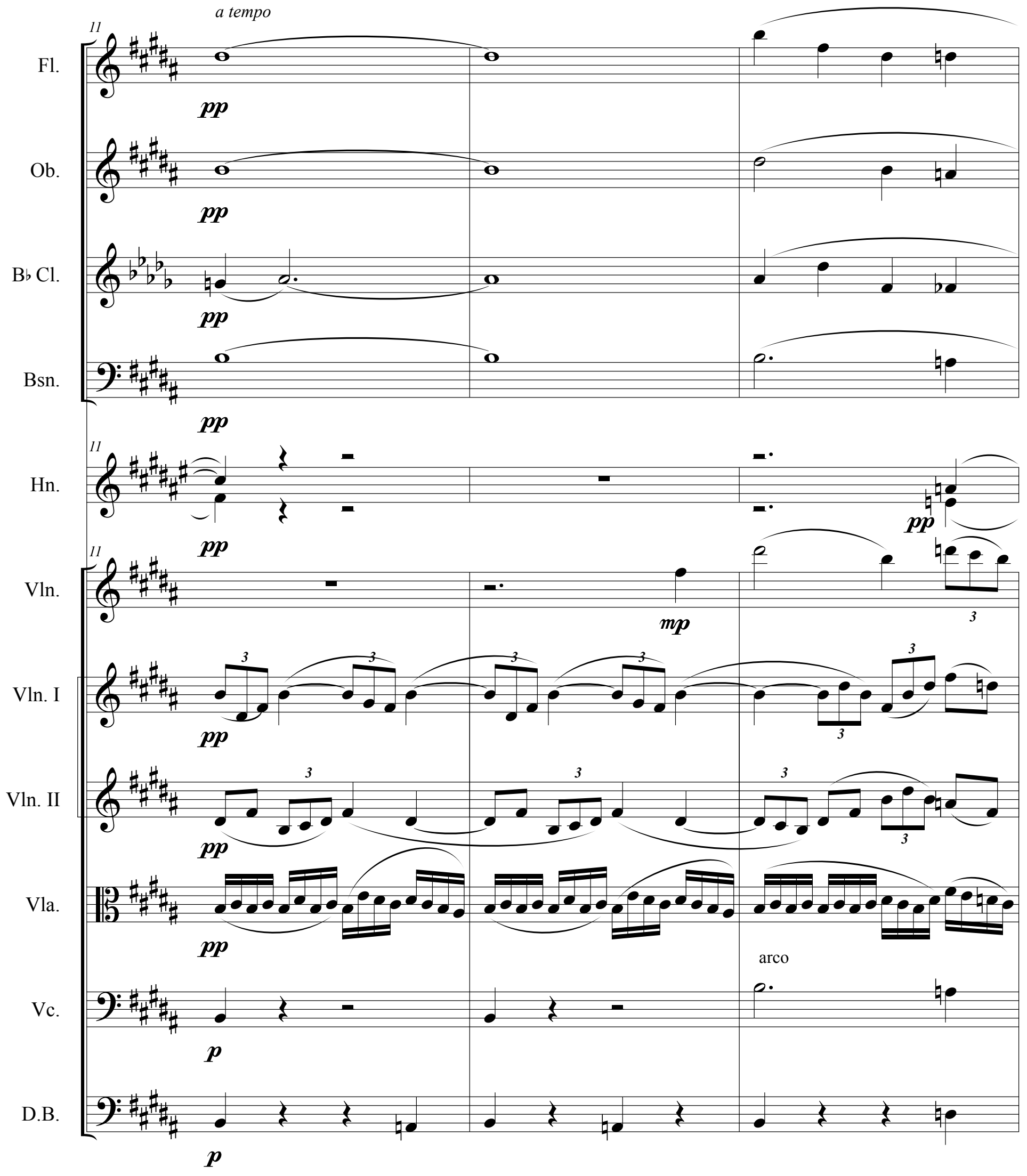




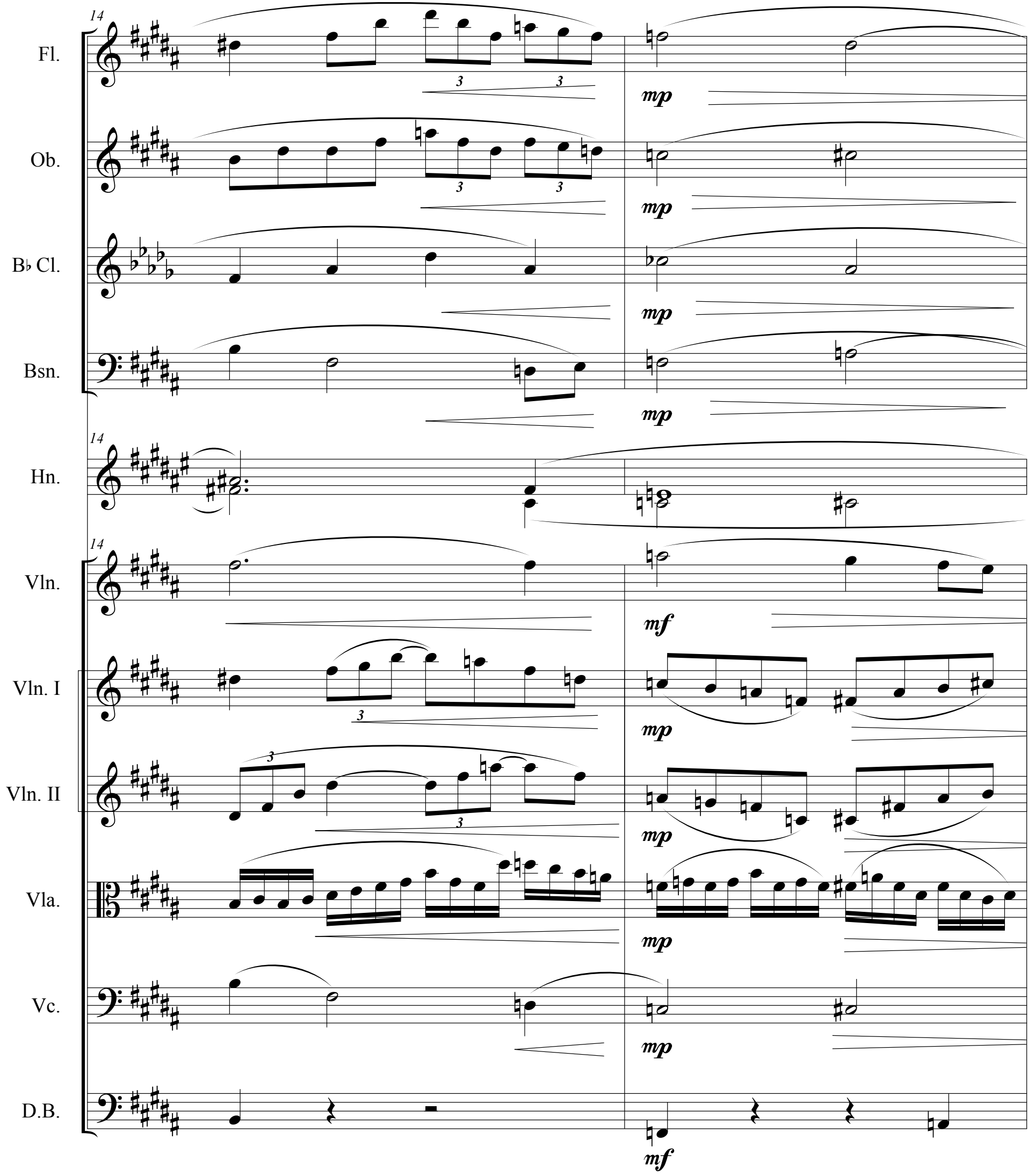


Score
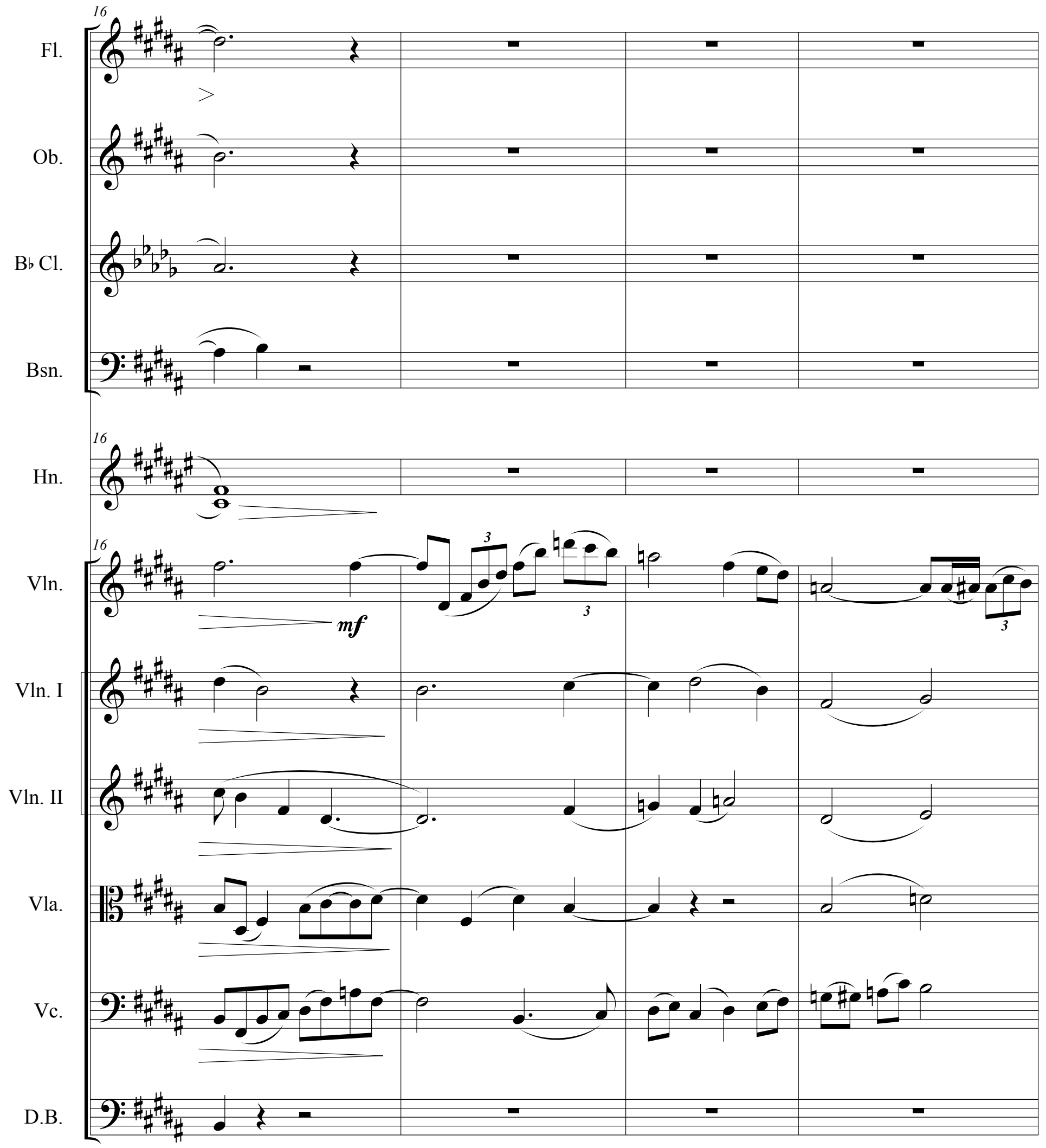

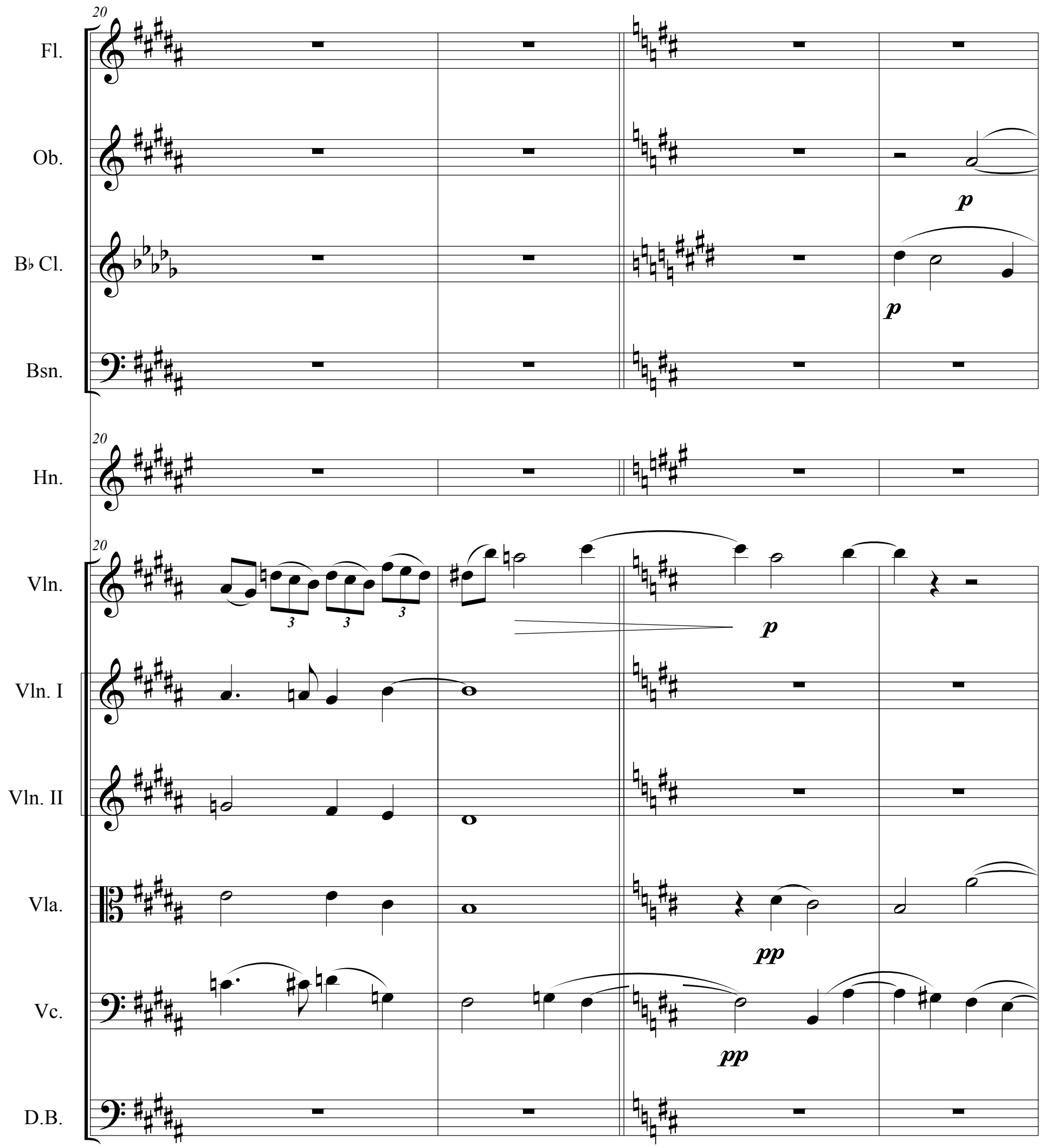
Score

For Violin Solo and Chamber Orchestra

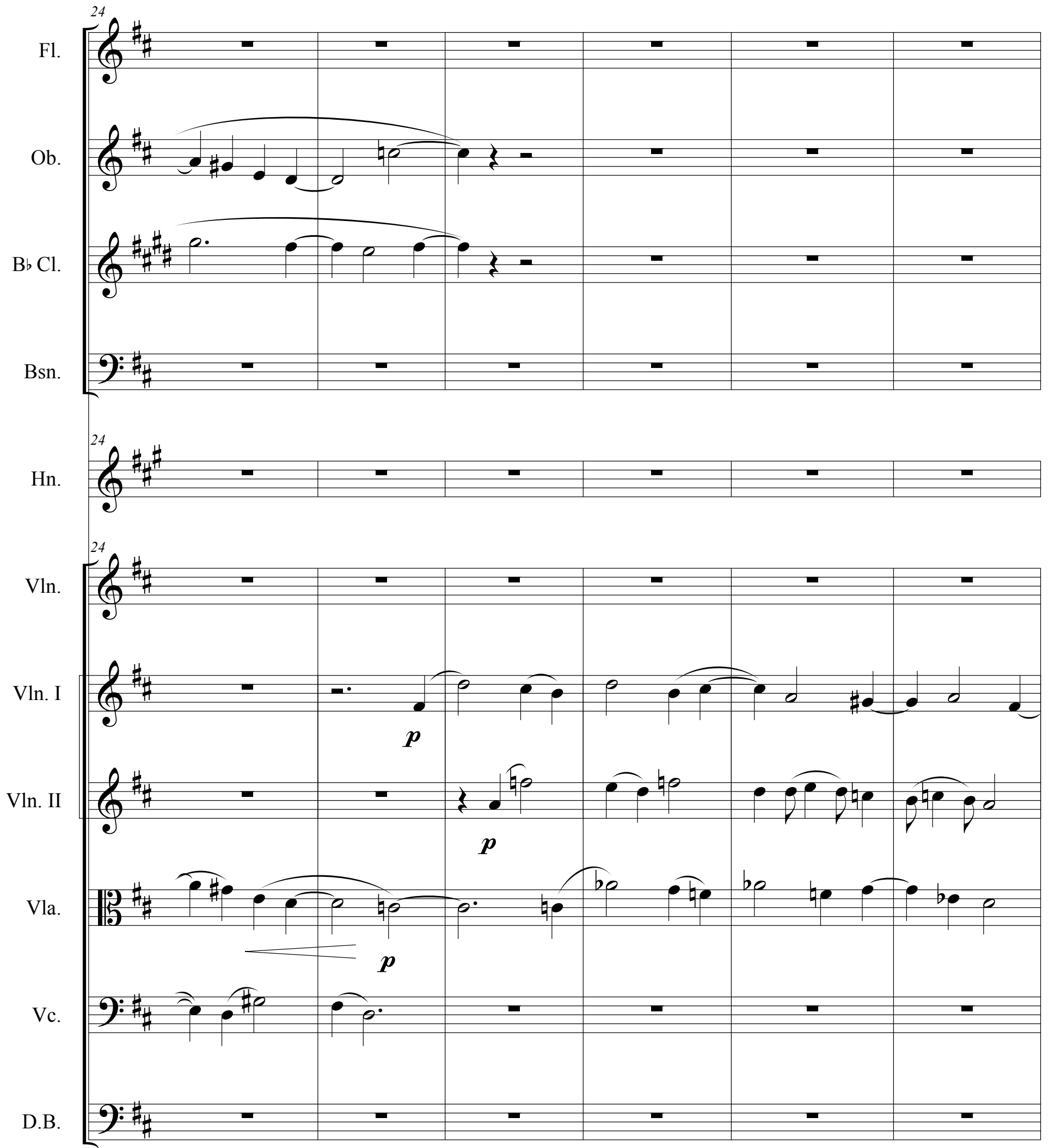


Fl.

$\mathrm{Ob}$.

$\mathrm{B} b \mathrm{Cl}$.

Bsn.

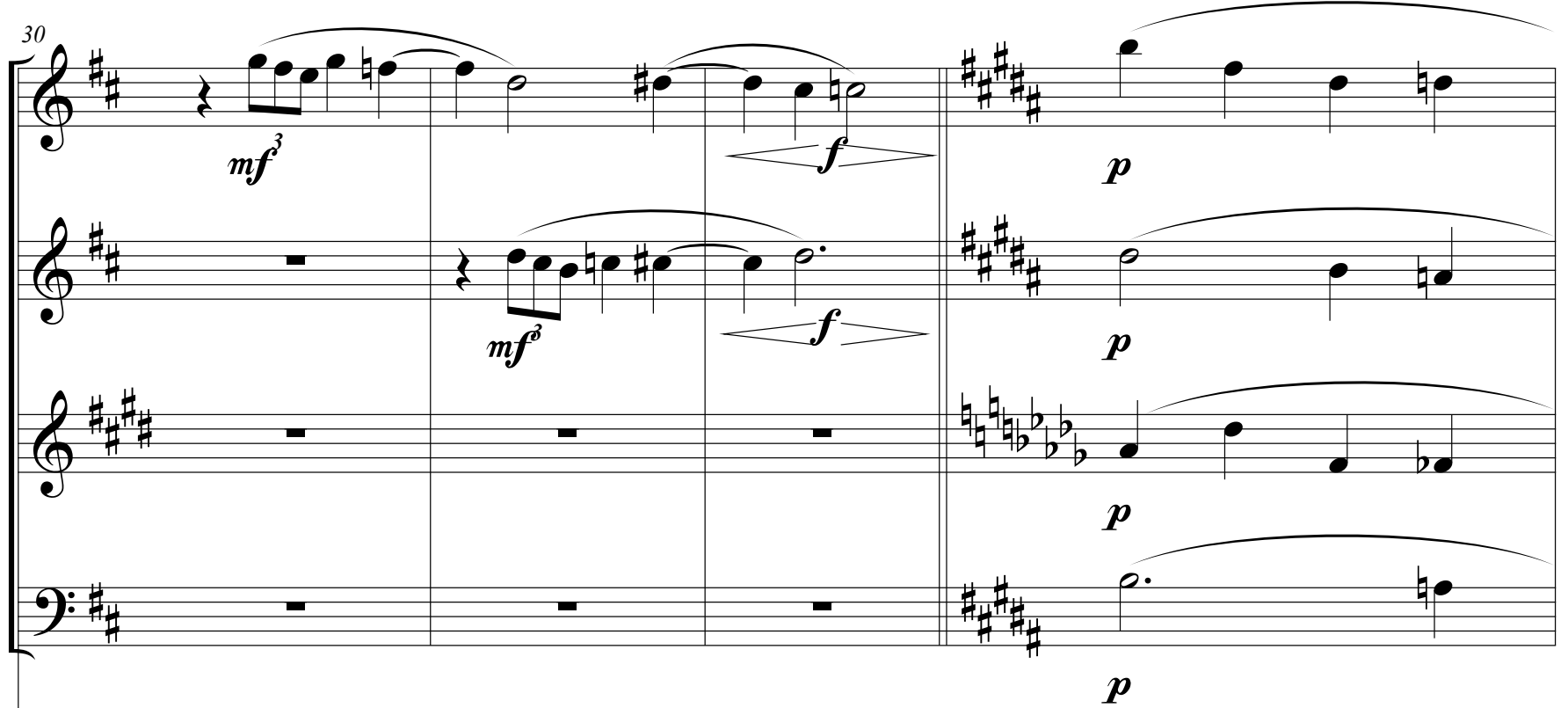

Hn.

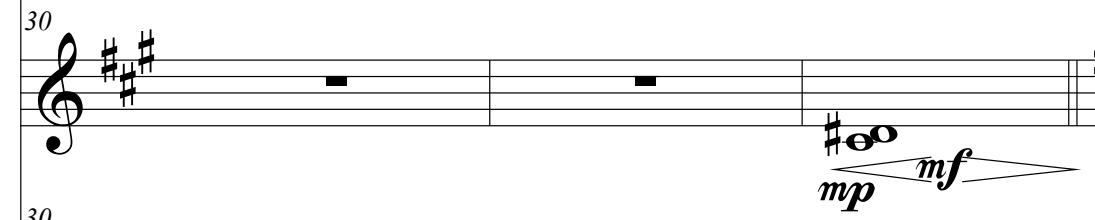

Vln.

Vln. I

Vln. II
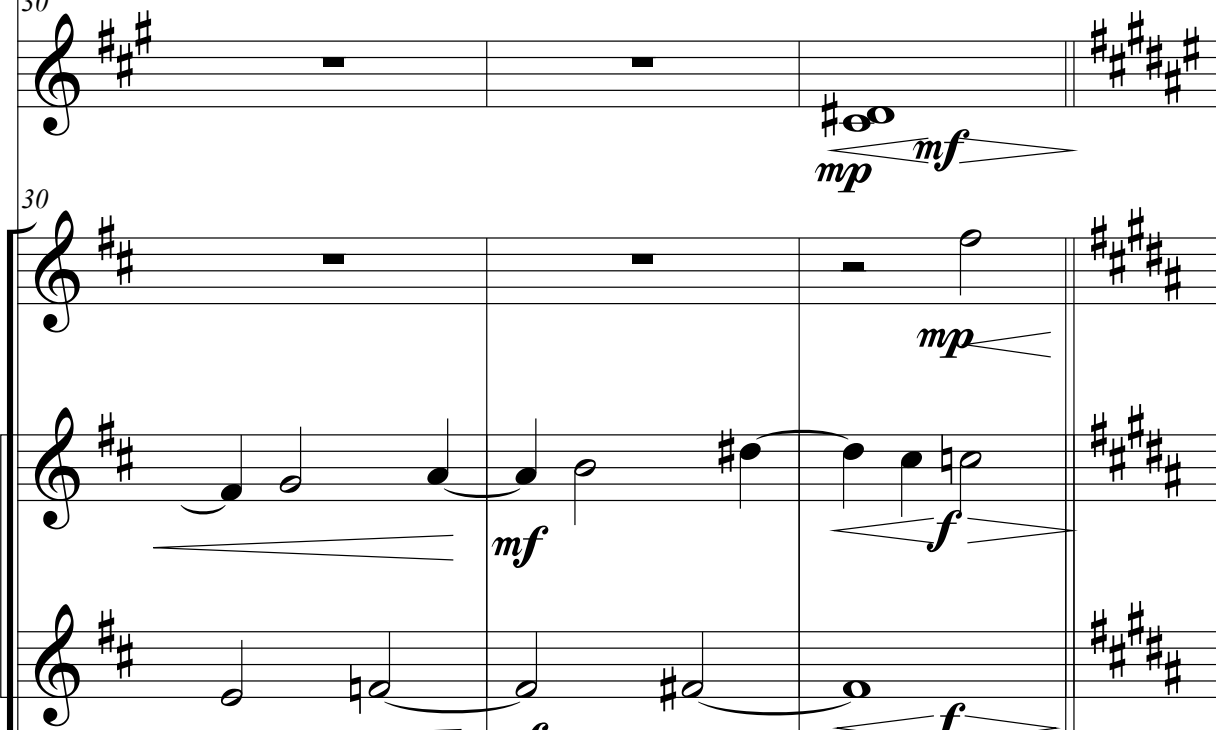

- 
Score

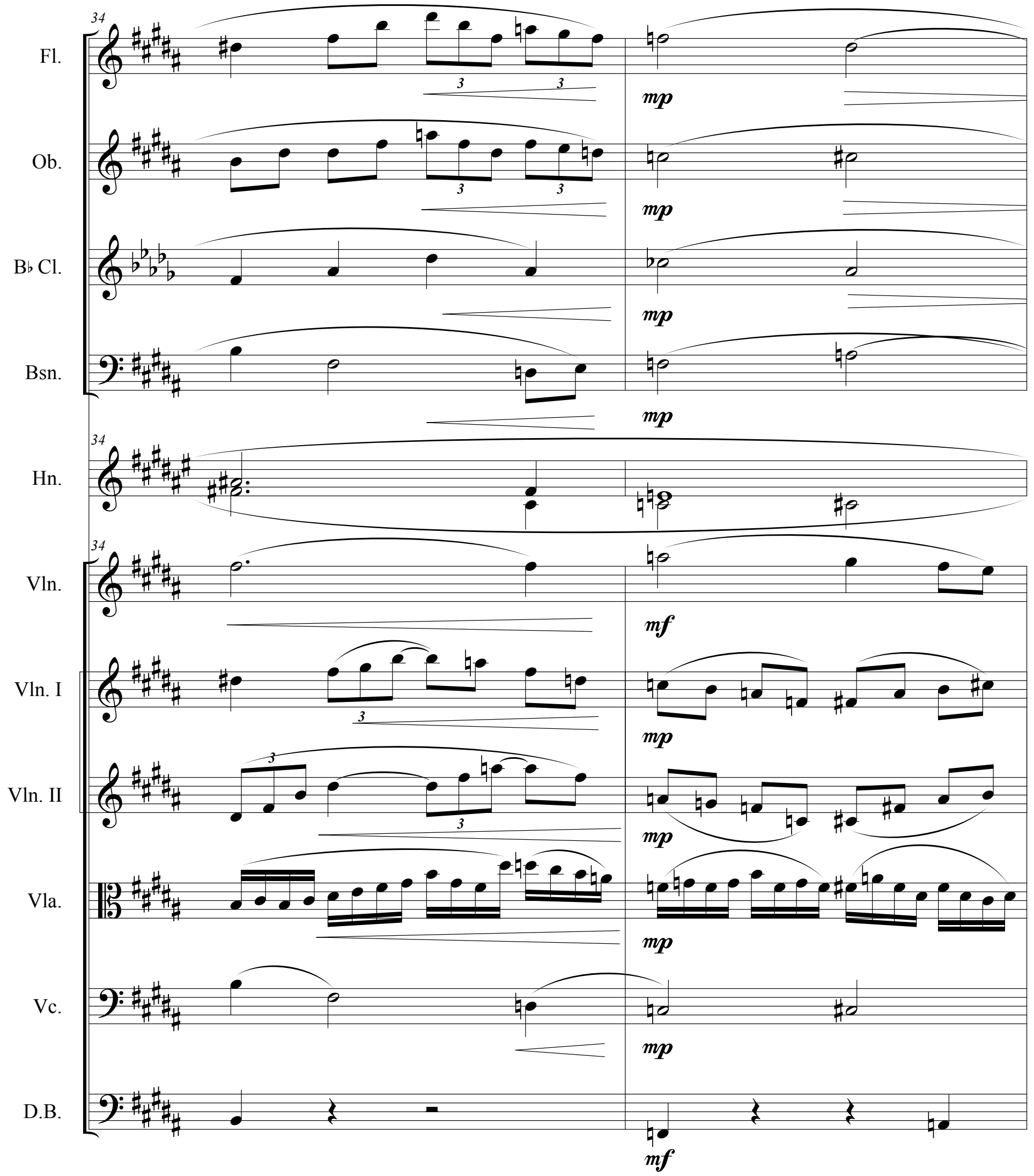




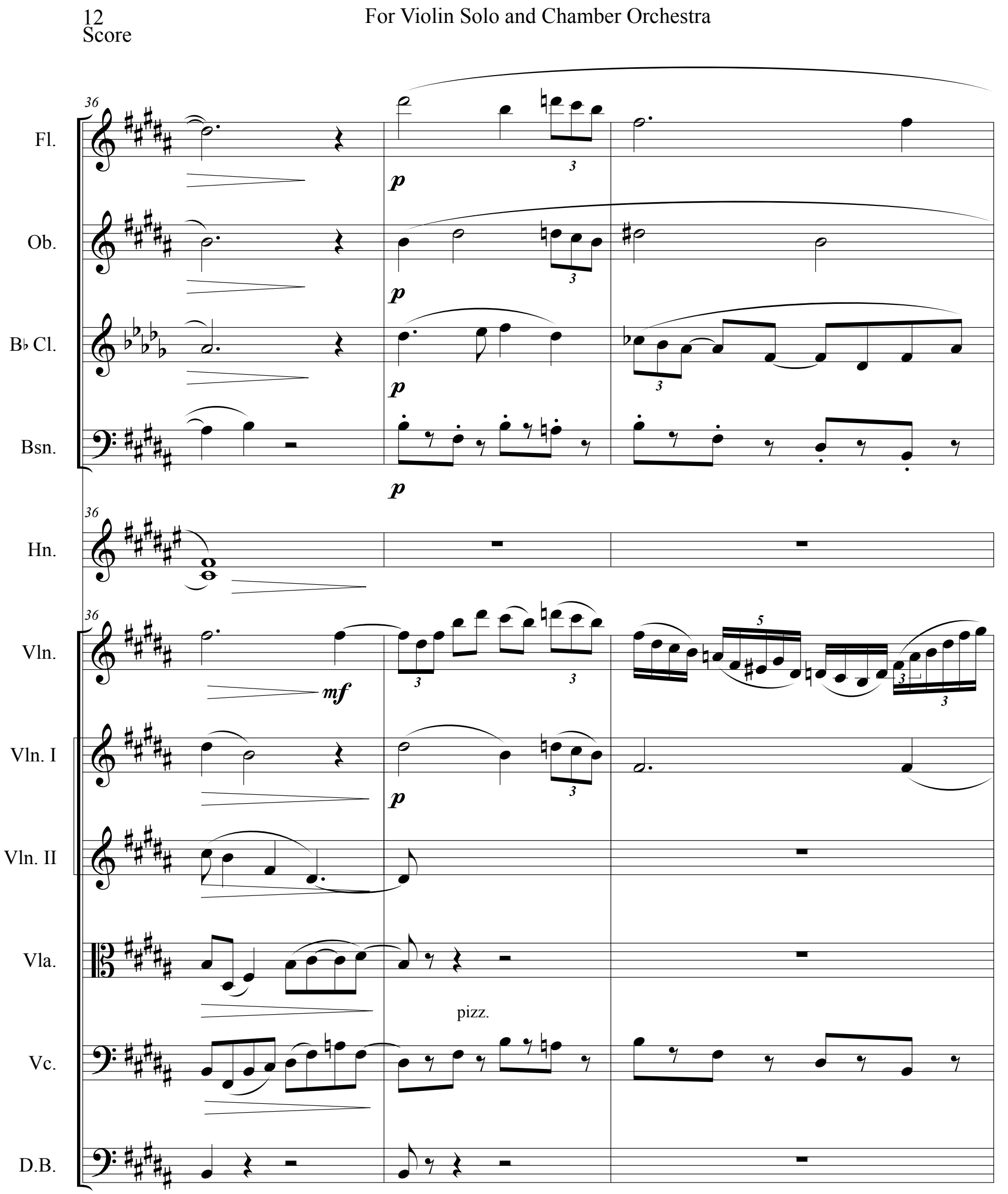


Score

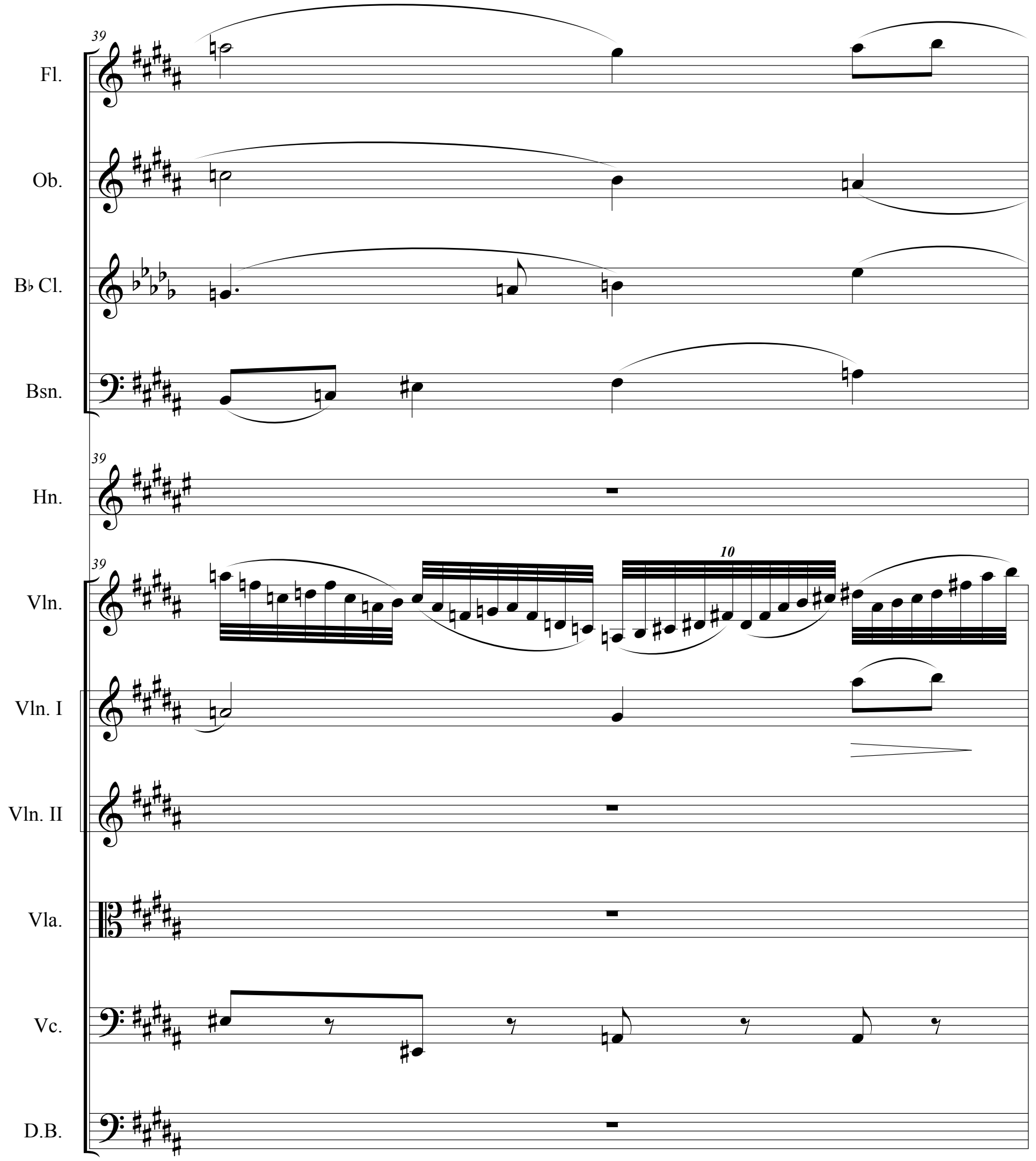


Fl.

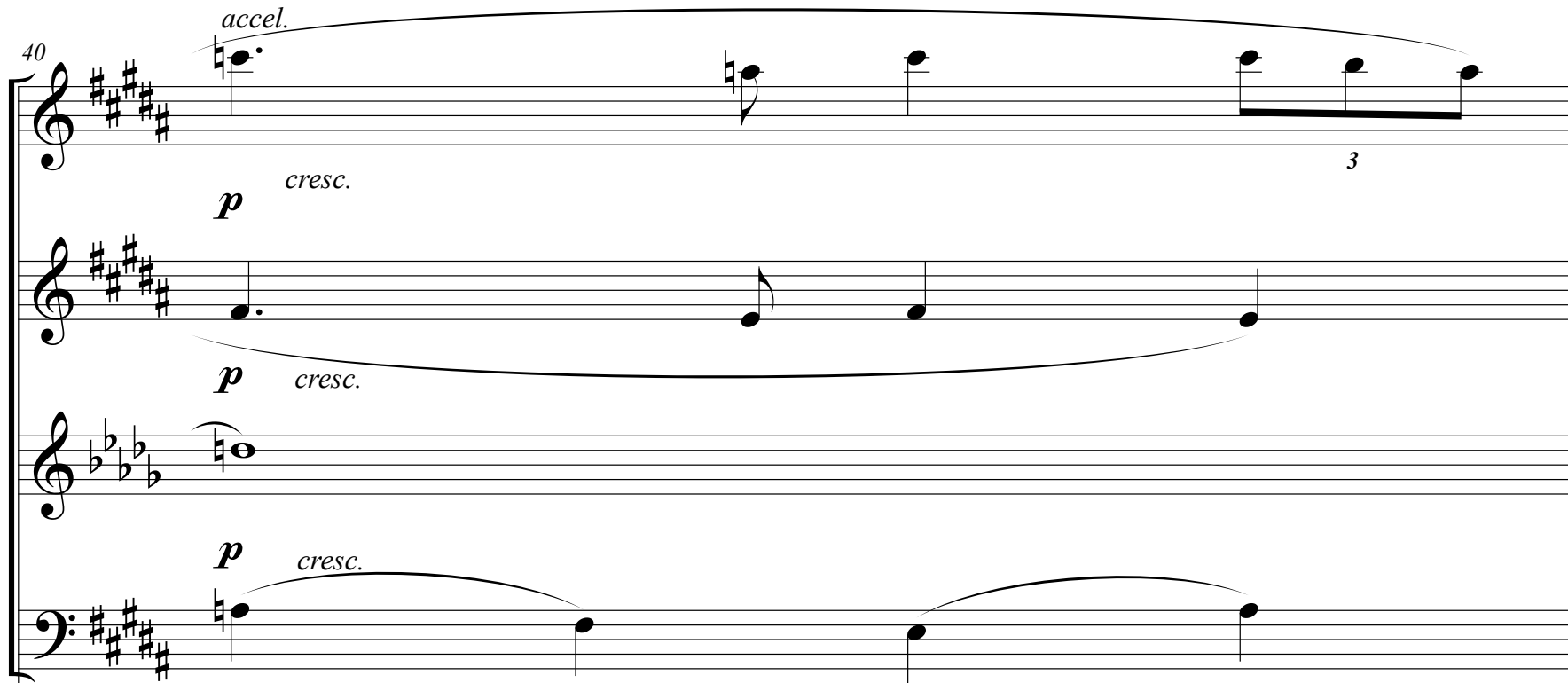

Bsn.

$\mathrm{Ob}$.

$\mathrm{B} b \mathrm{Cl}$.

$$
\text { Bsin. }
$$

$$
{ }_{40} \boldsymbol{p} \text { cresc. }
$$$$
\text { Hn. }
$$

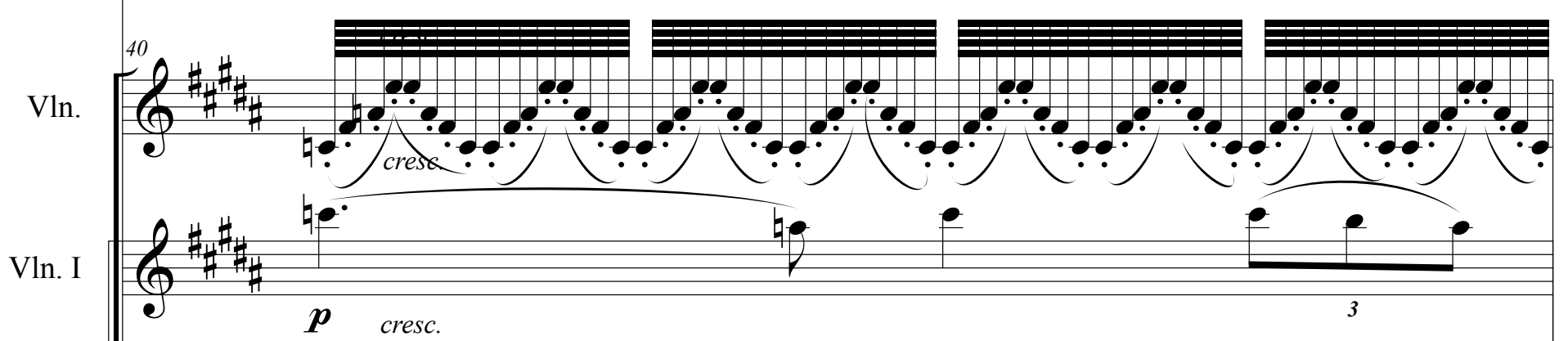

Vln. II
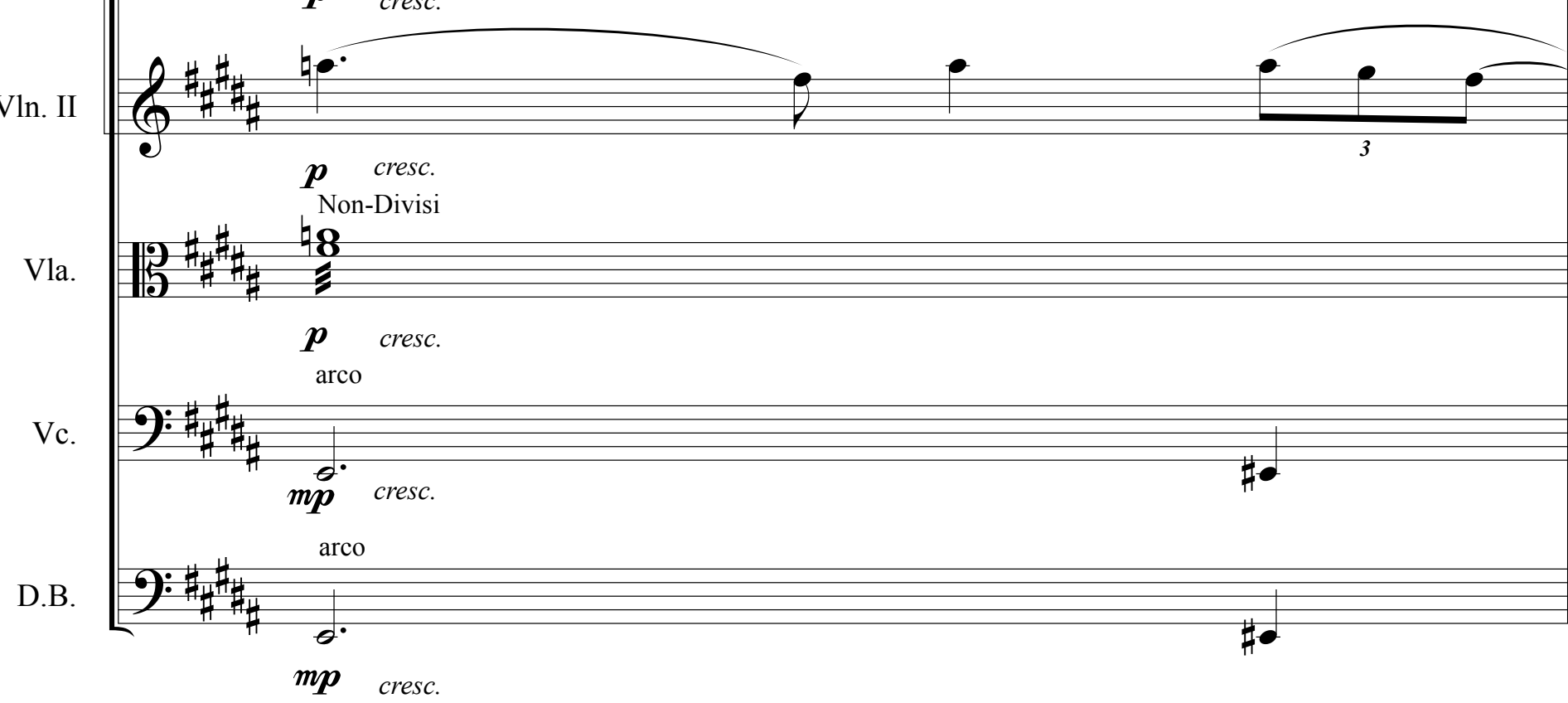


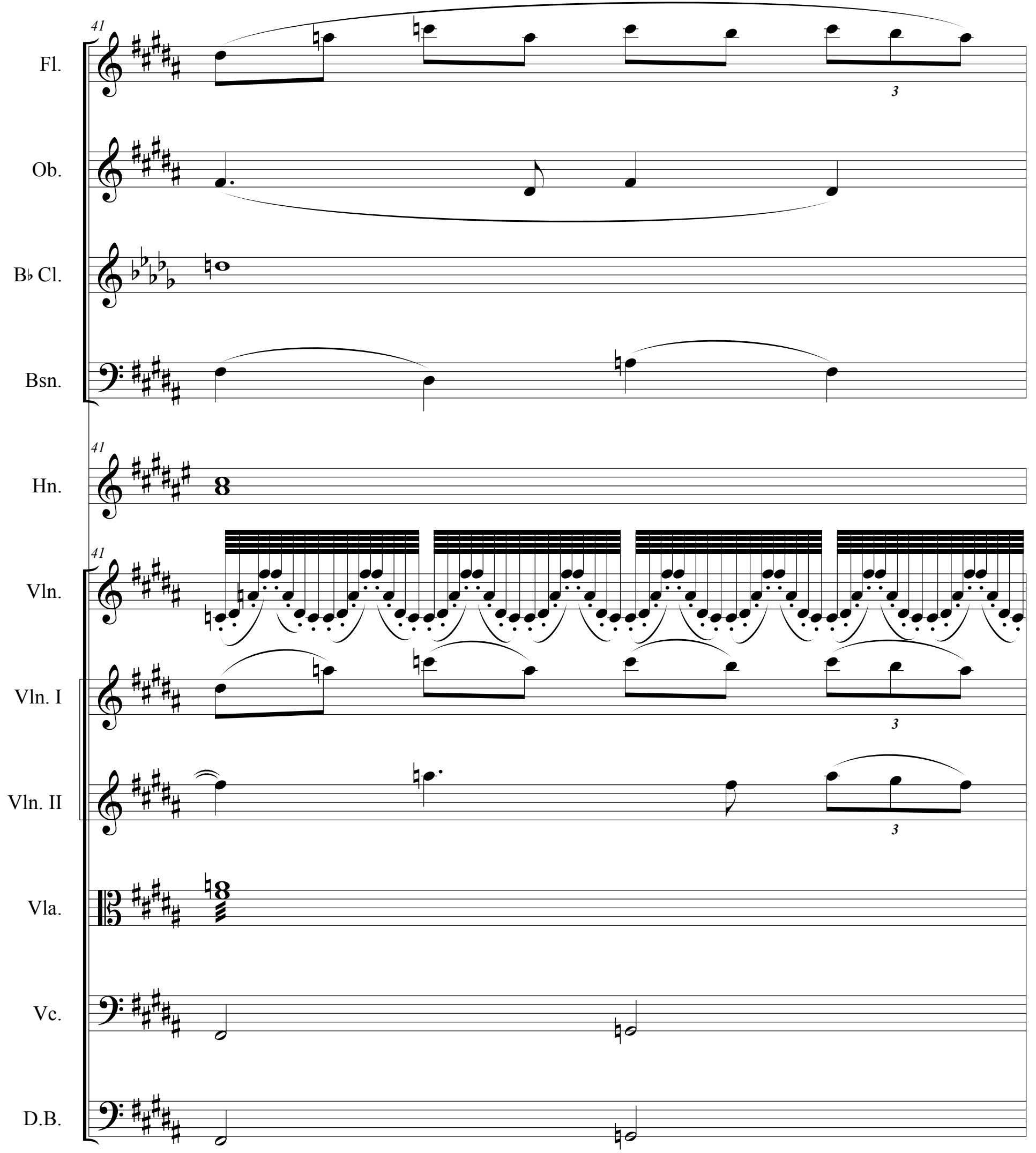




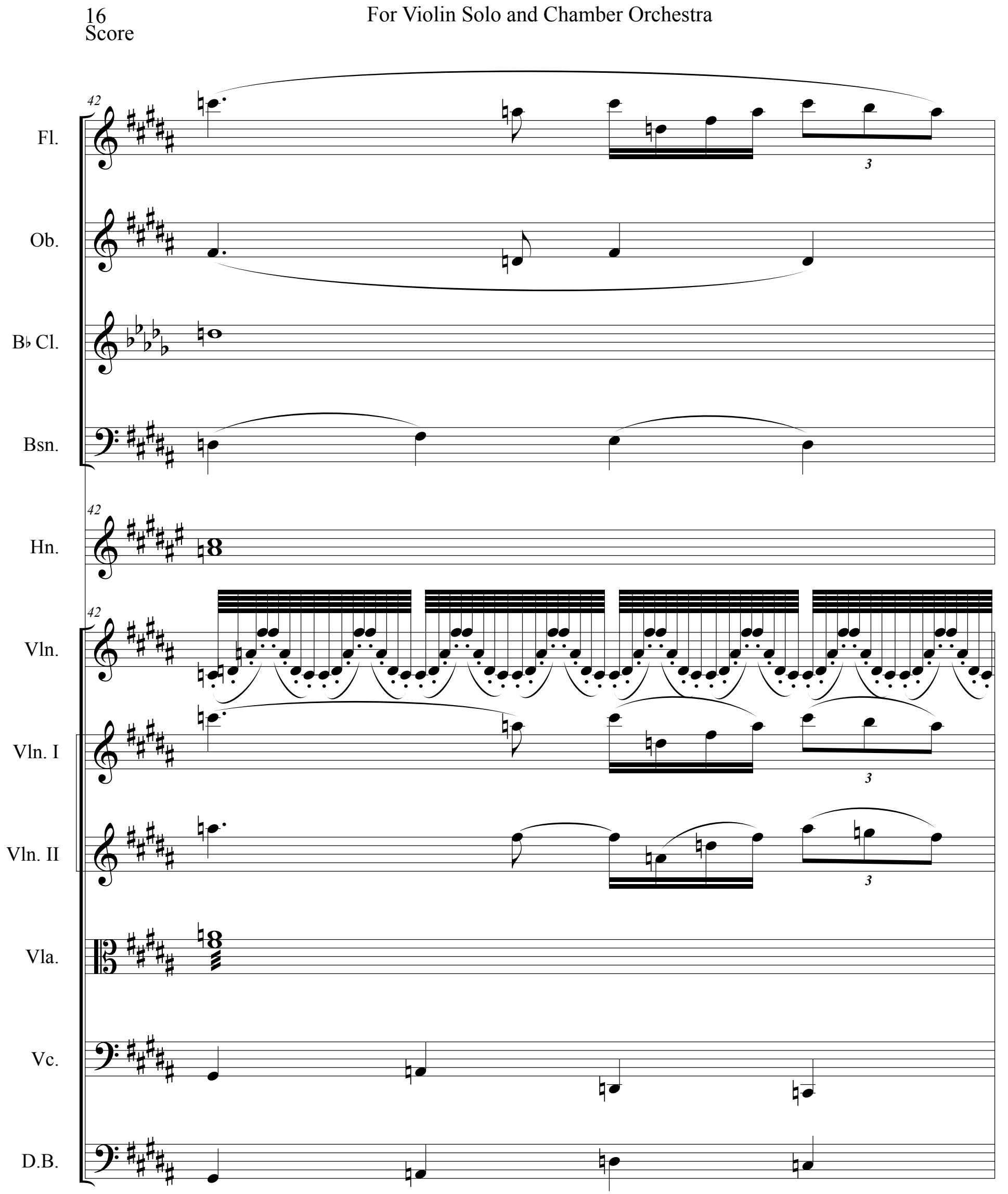


For Violin Solo and Chamber Orchestra

17

Score

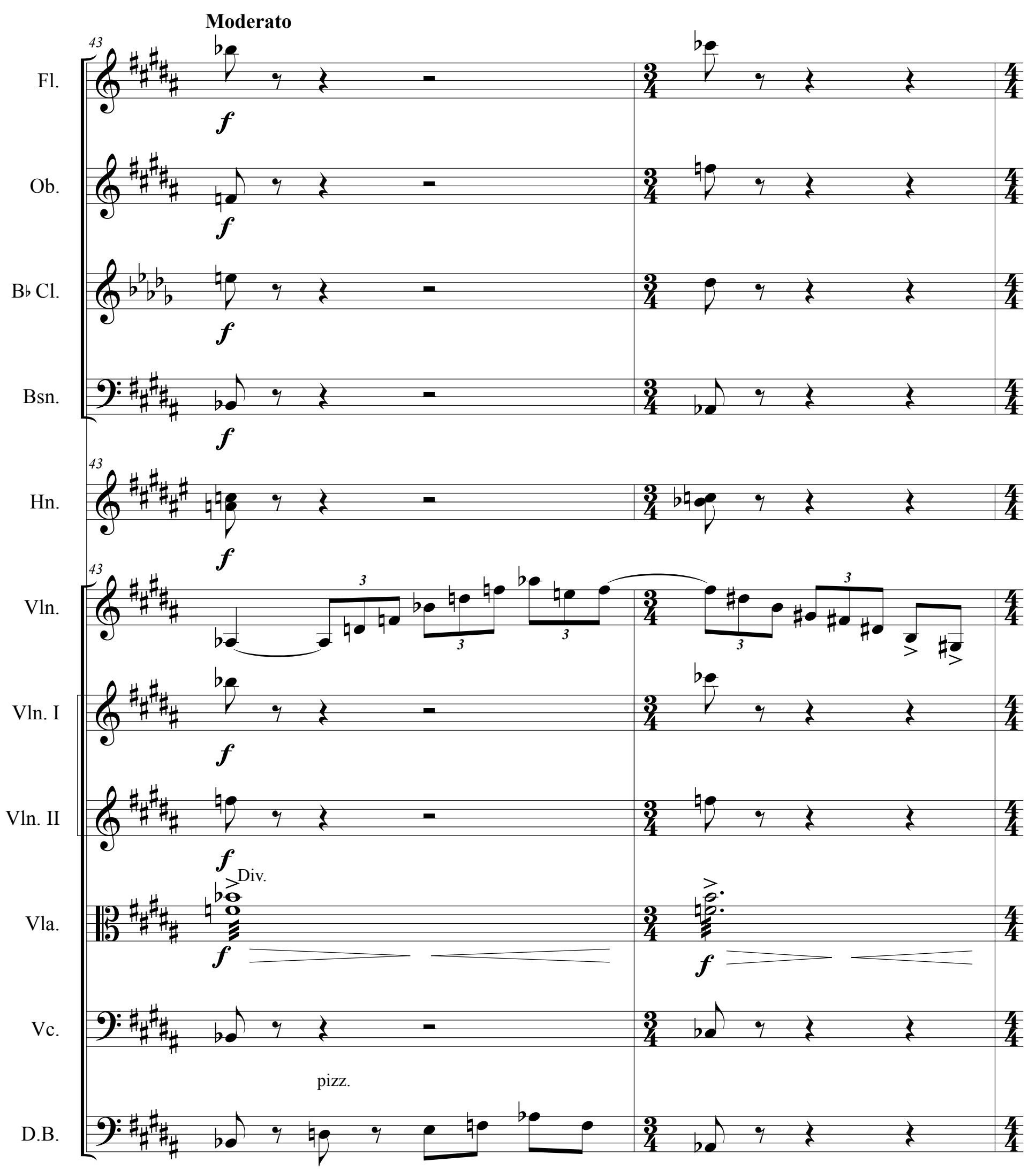


18

For Violin Solo and Chamber Orchestra

Fl.

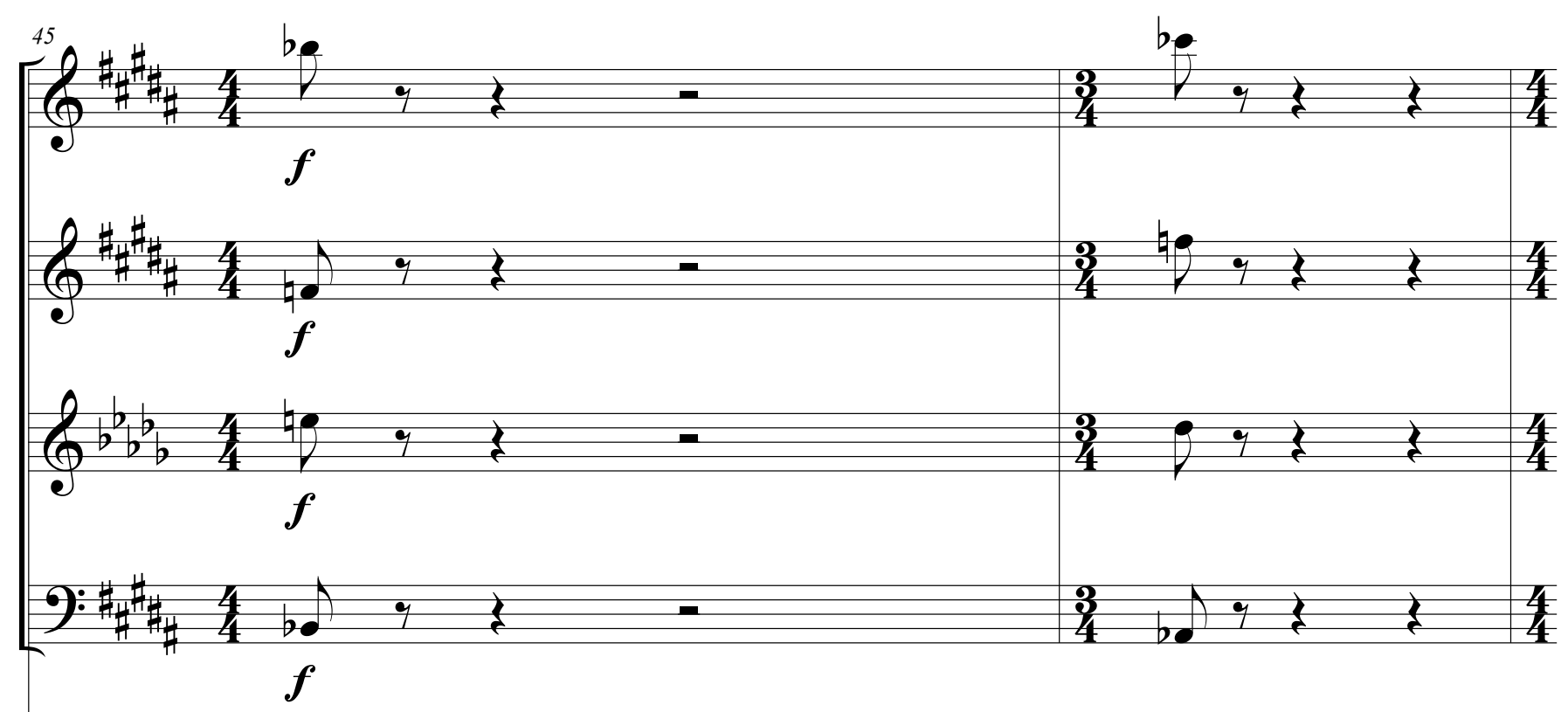

Hon.

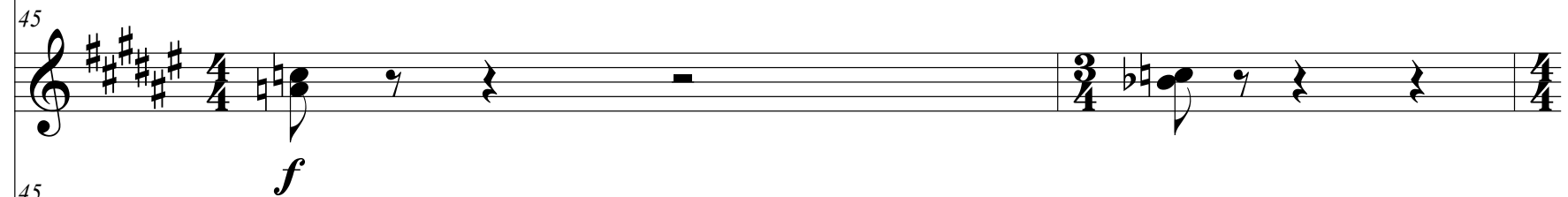

Vln.

Ven. I

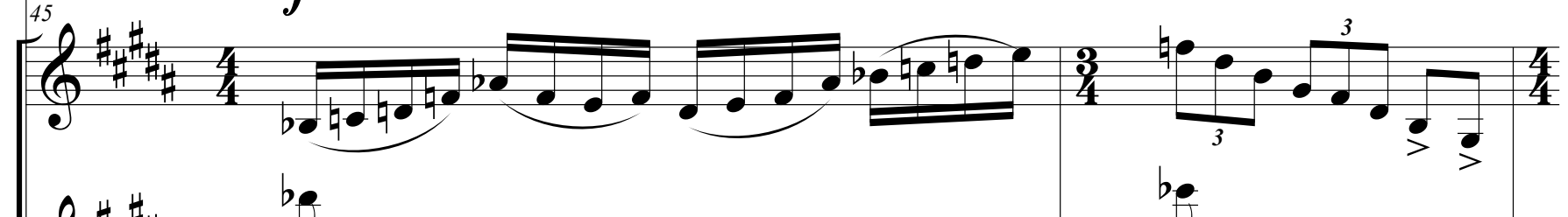

Vln. II

VIa.

Vc.

D.B.

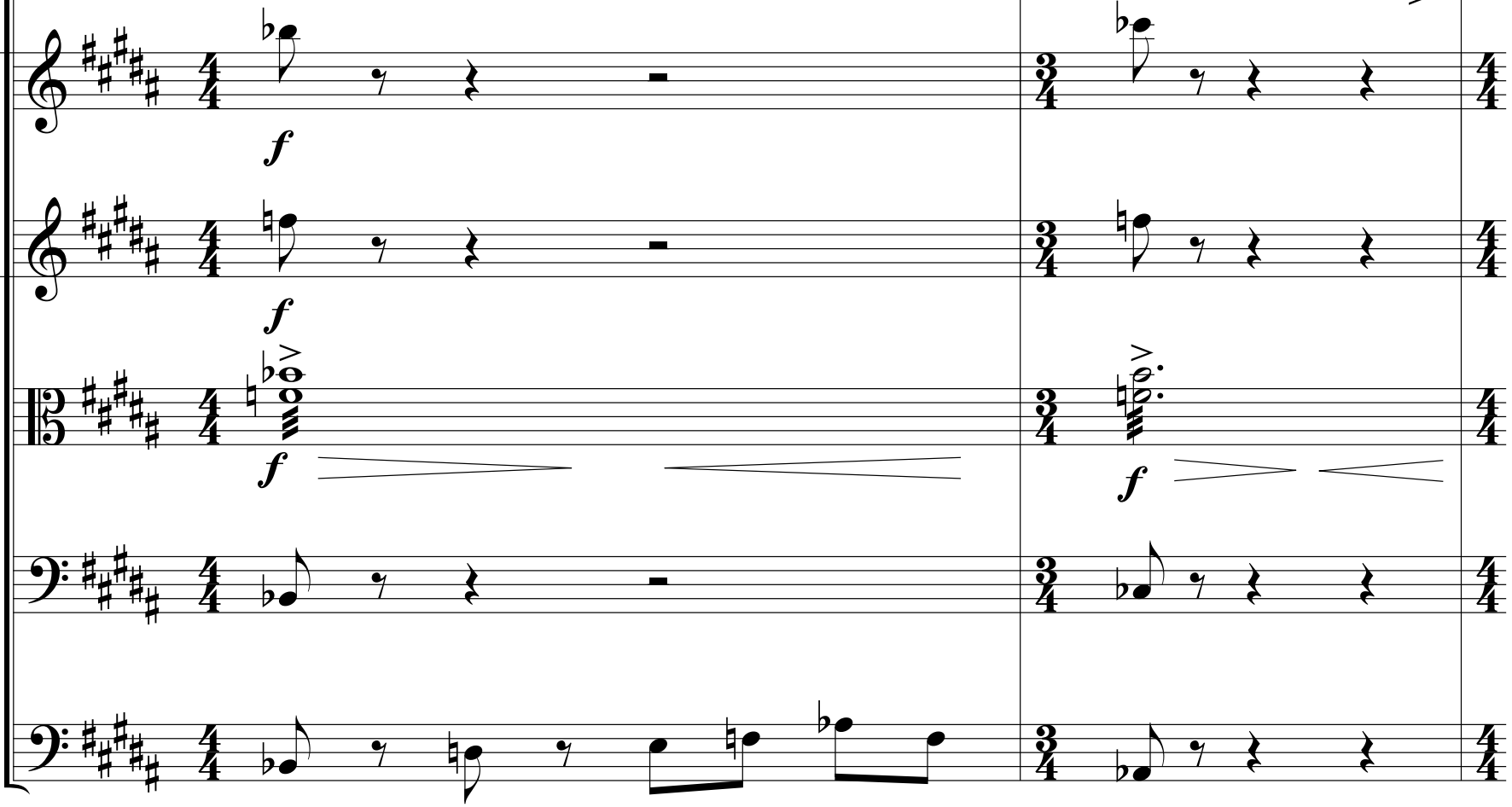




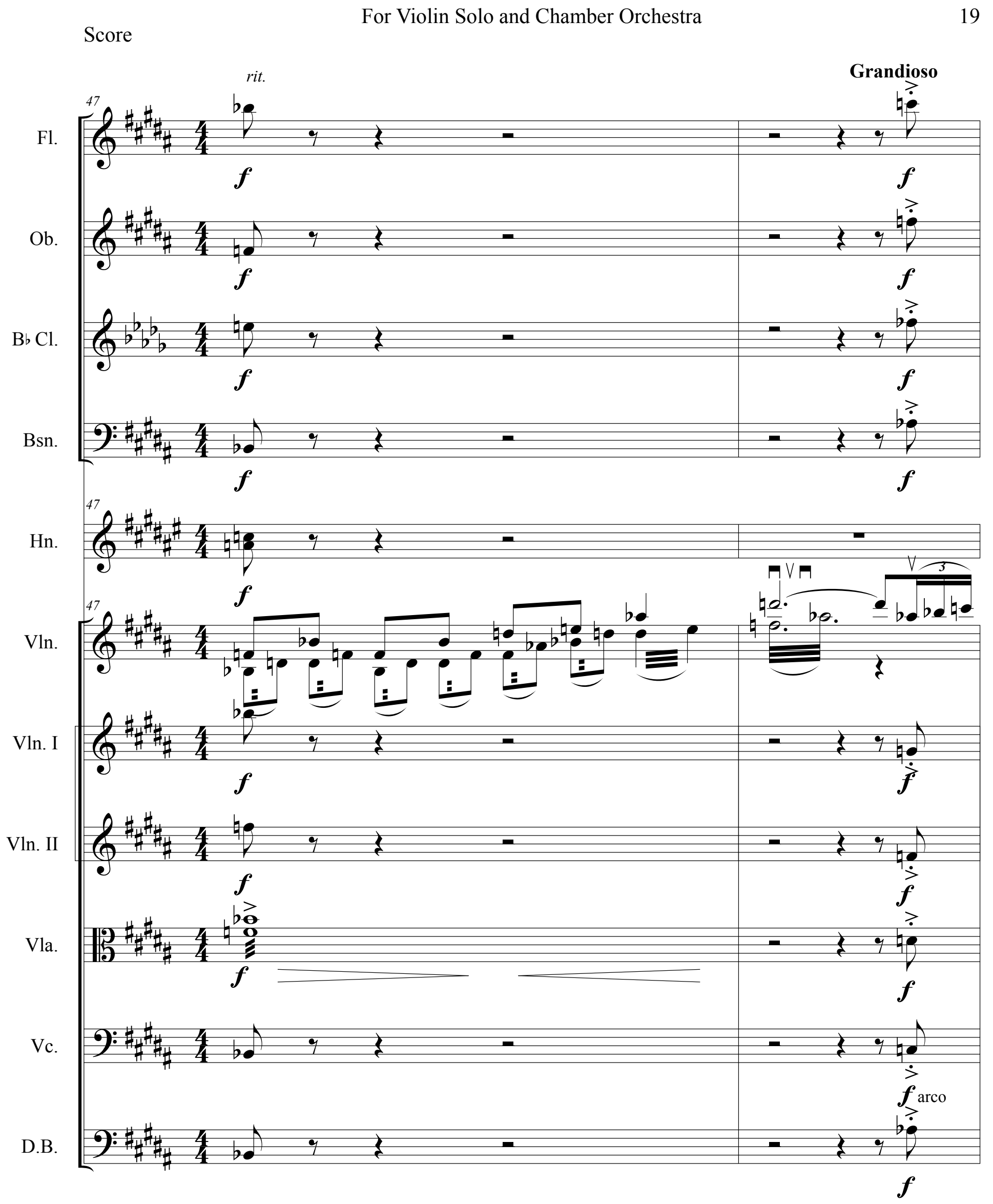




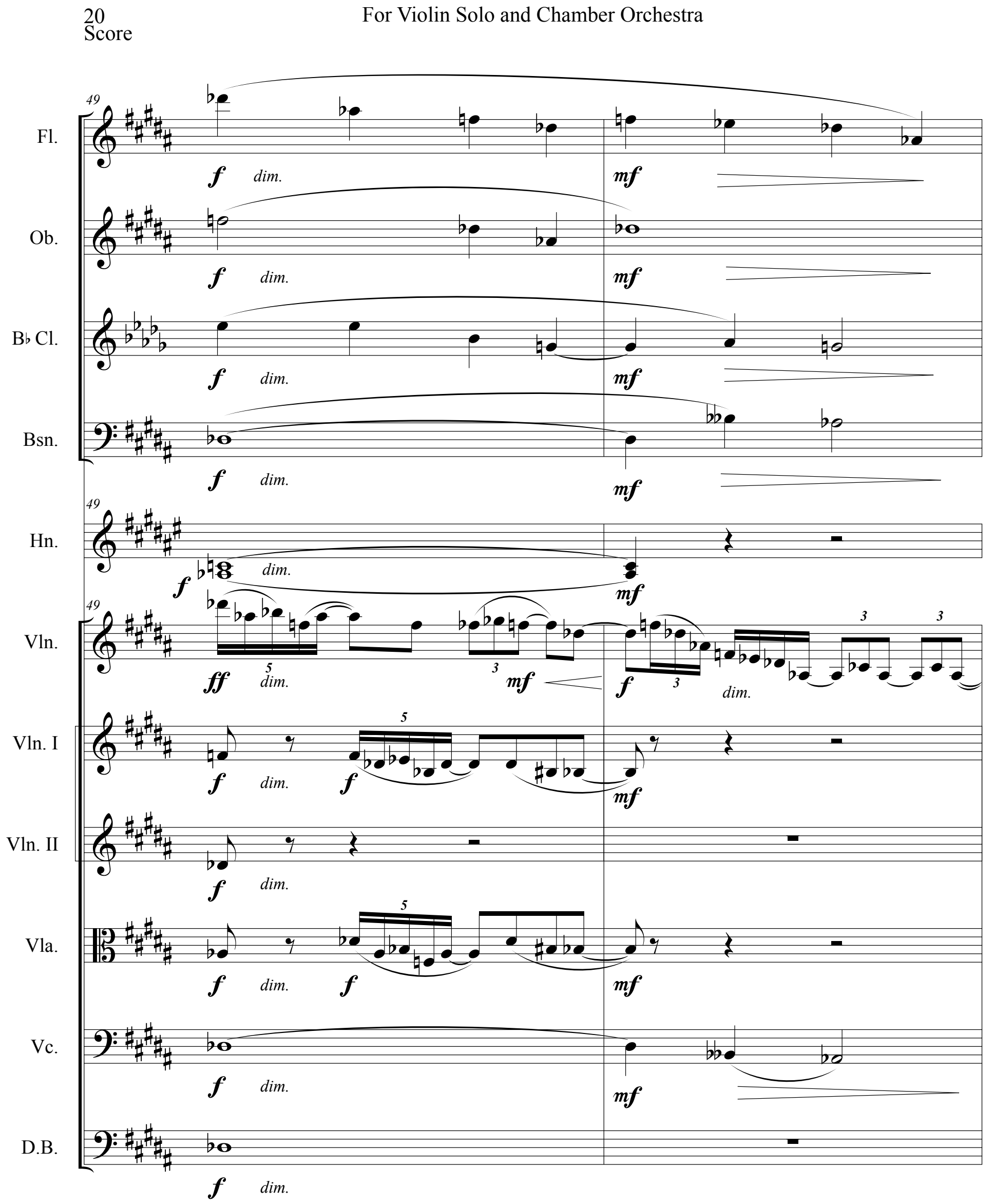


Score

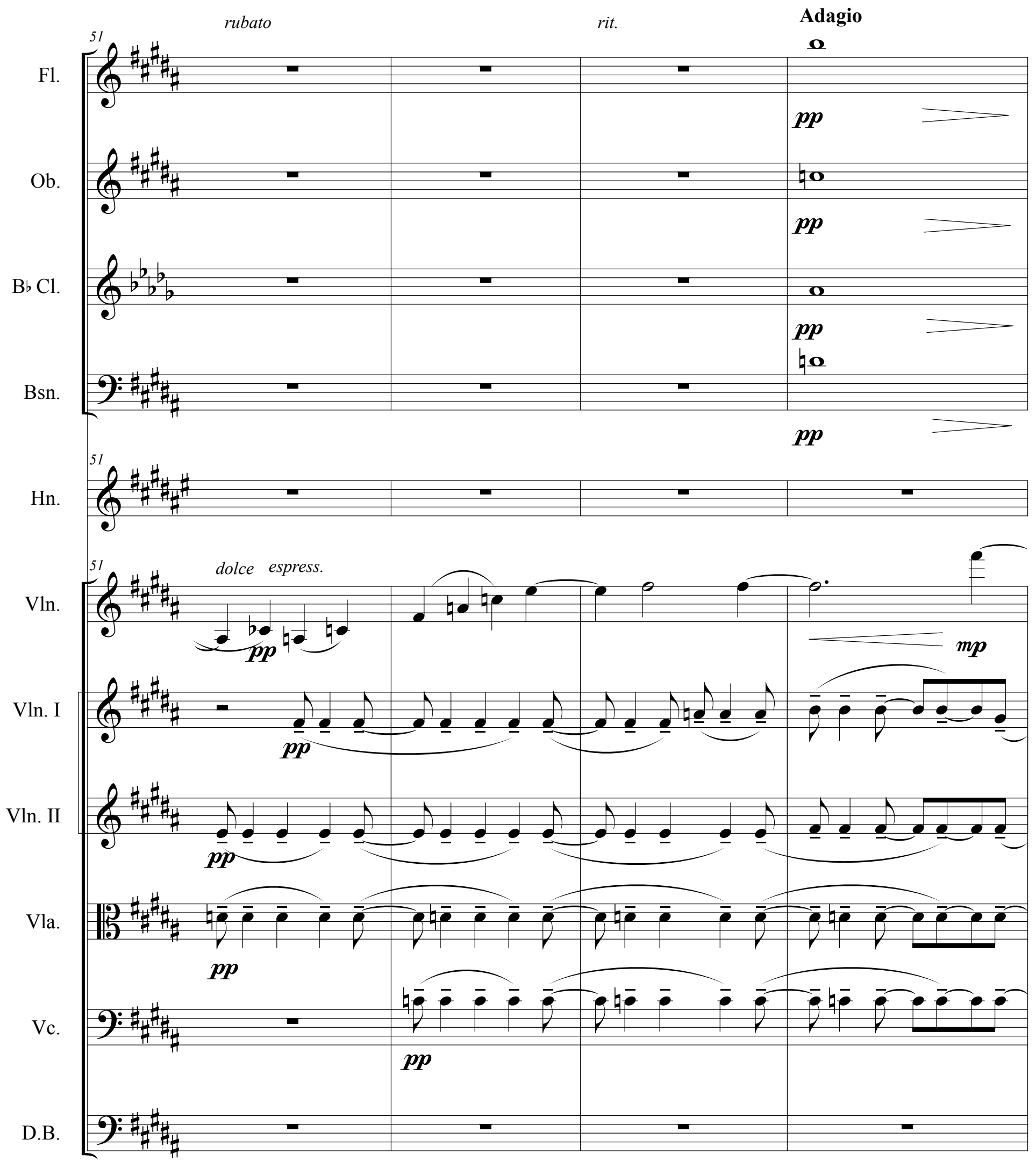




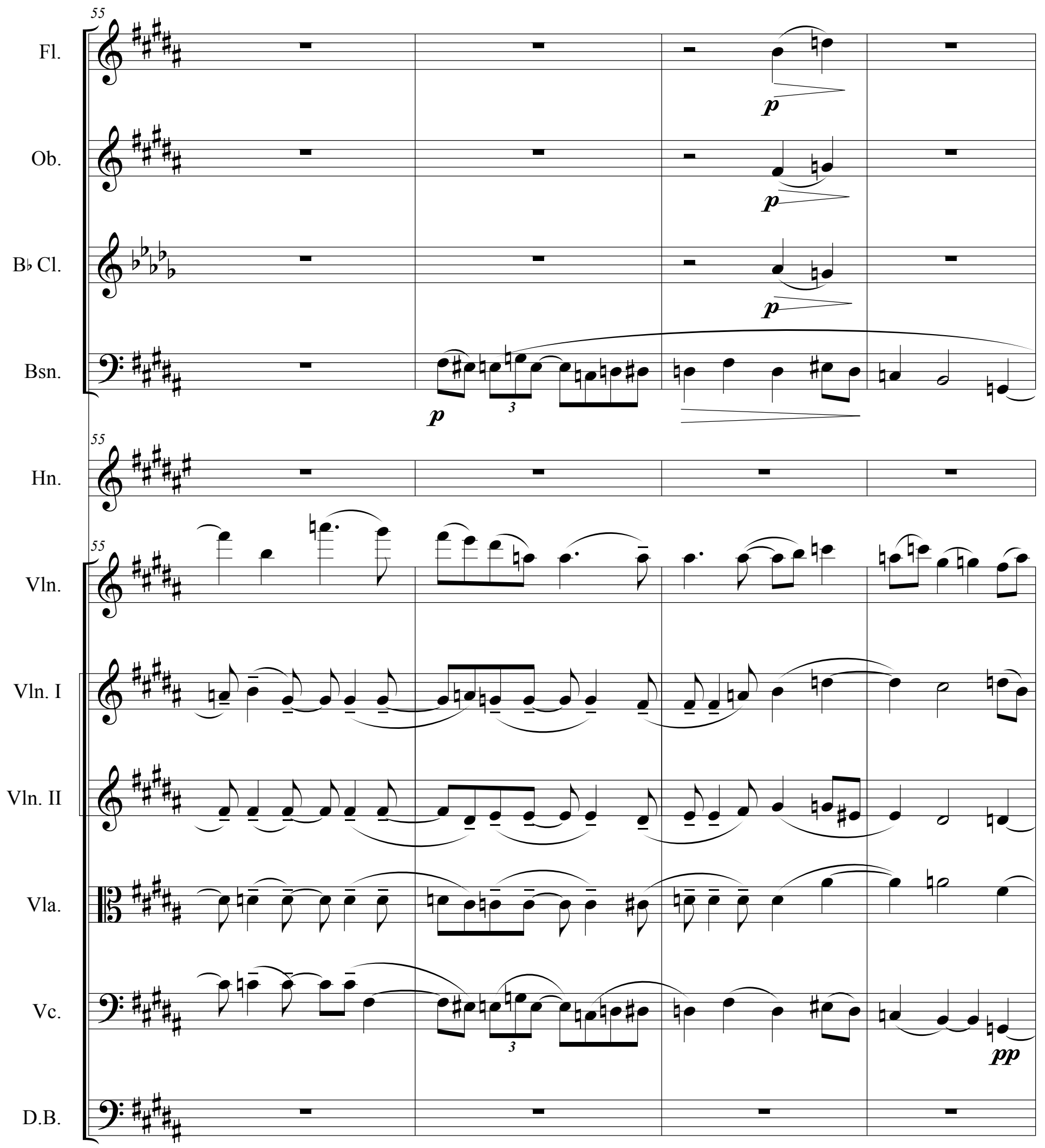


Score

For Violin Solo and Chamber Orchestra

23

Quarter $=134$

Allegro

Fl.

rit.

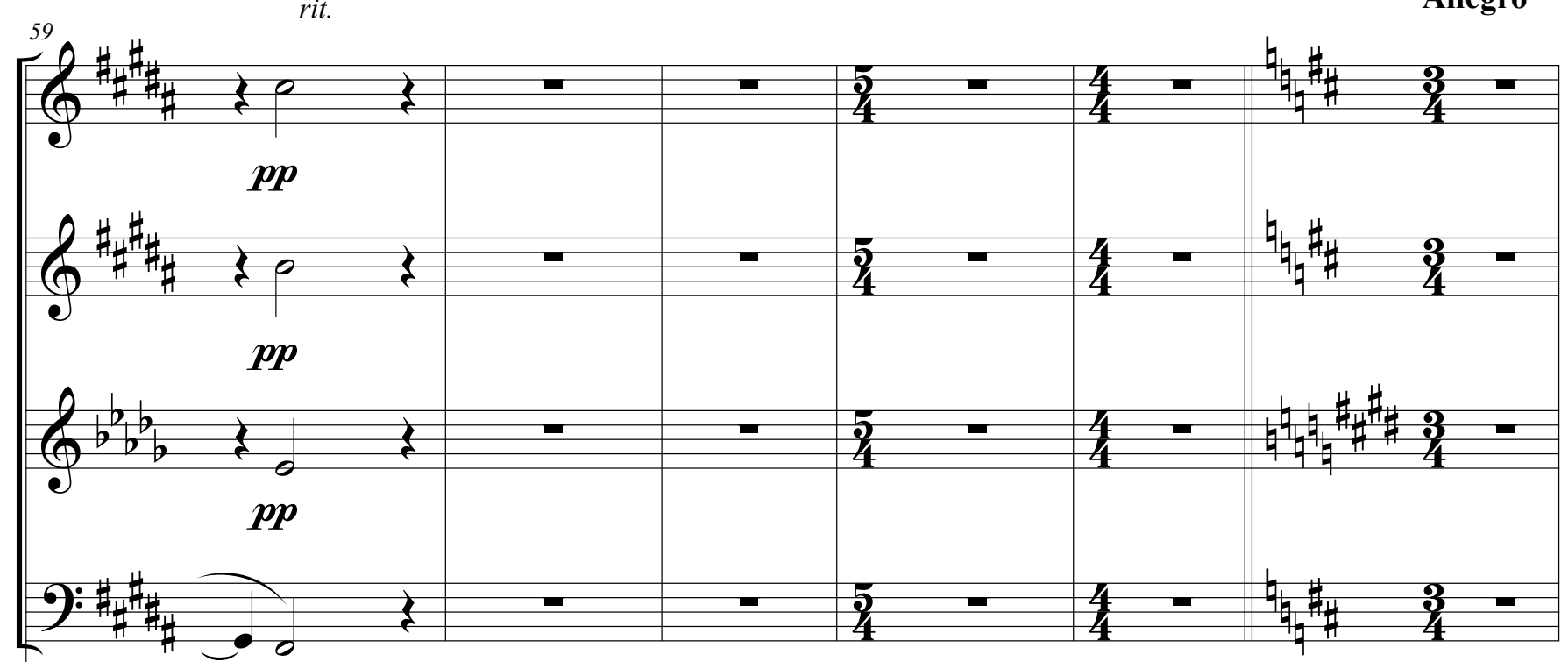

$\mathrm{B} b \mathrm{Cl}$.

Ob.

Bn.

Hr.

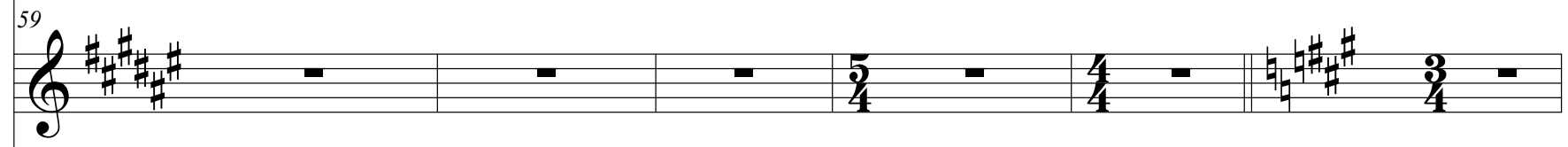

Vln.

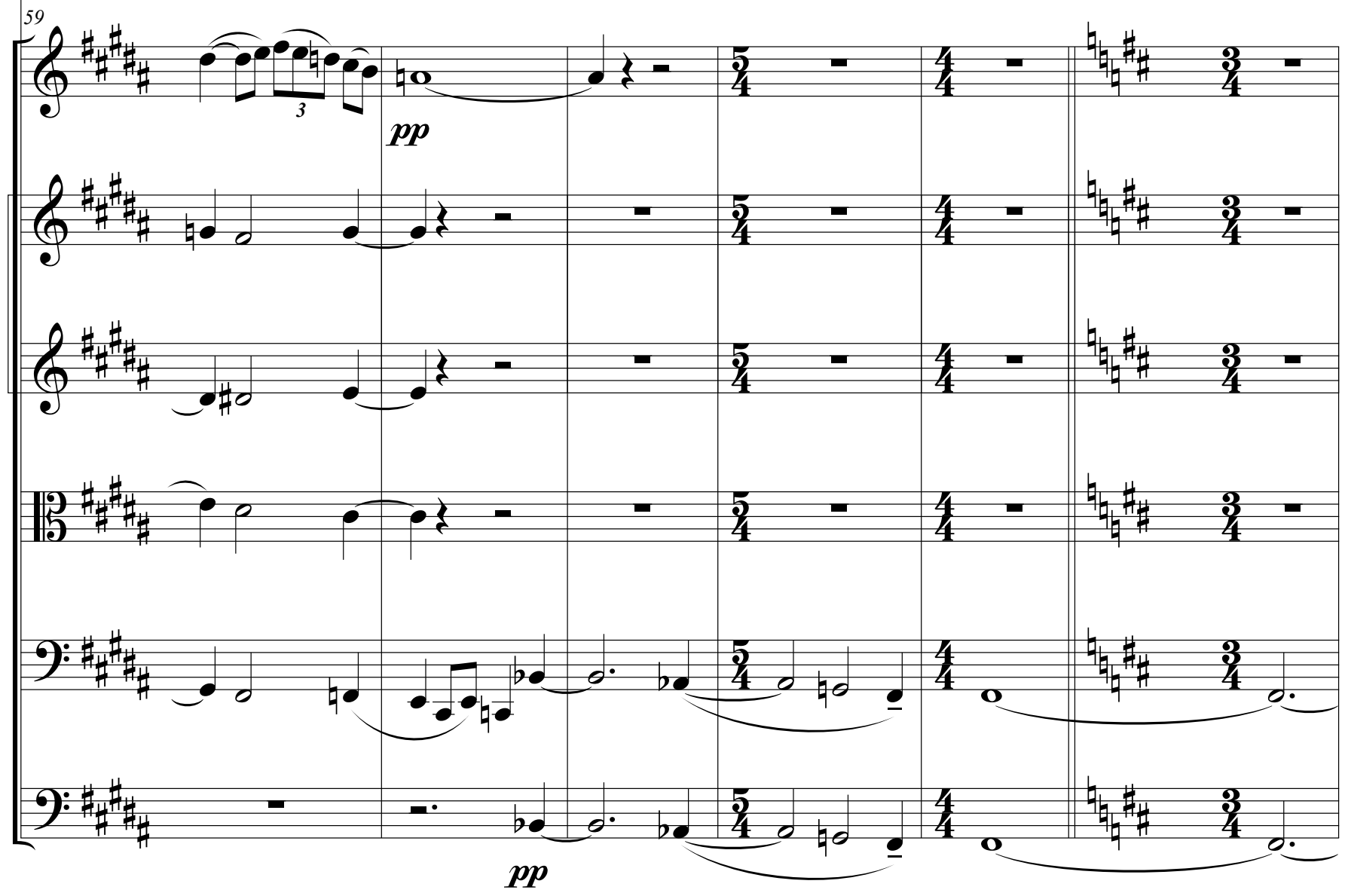



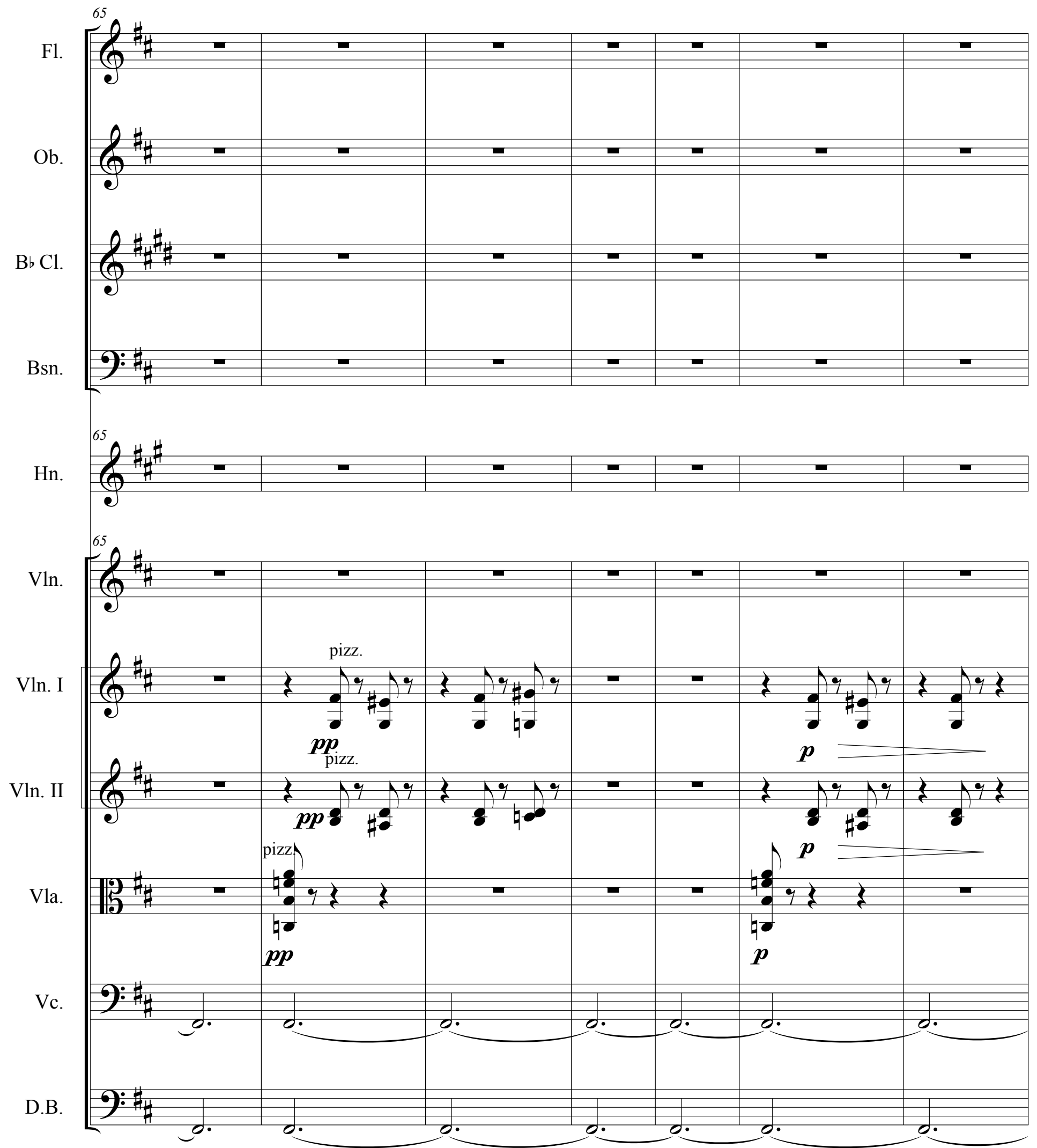
Score
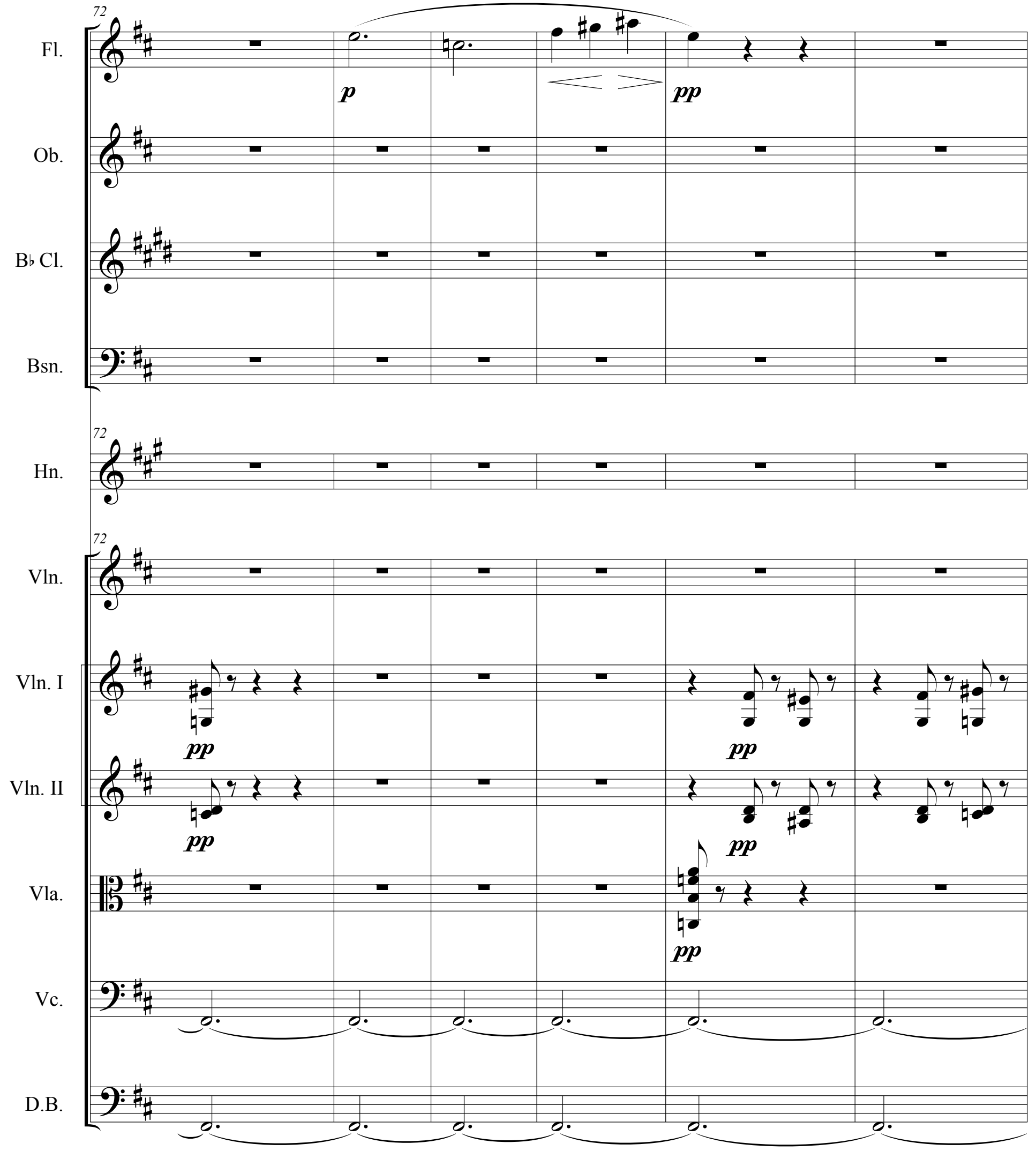

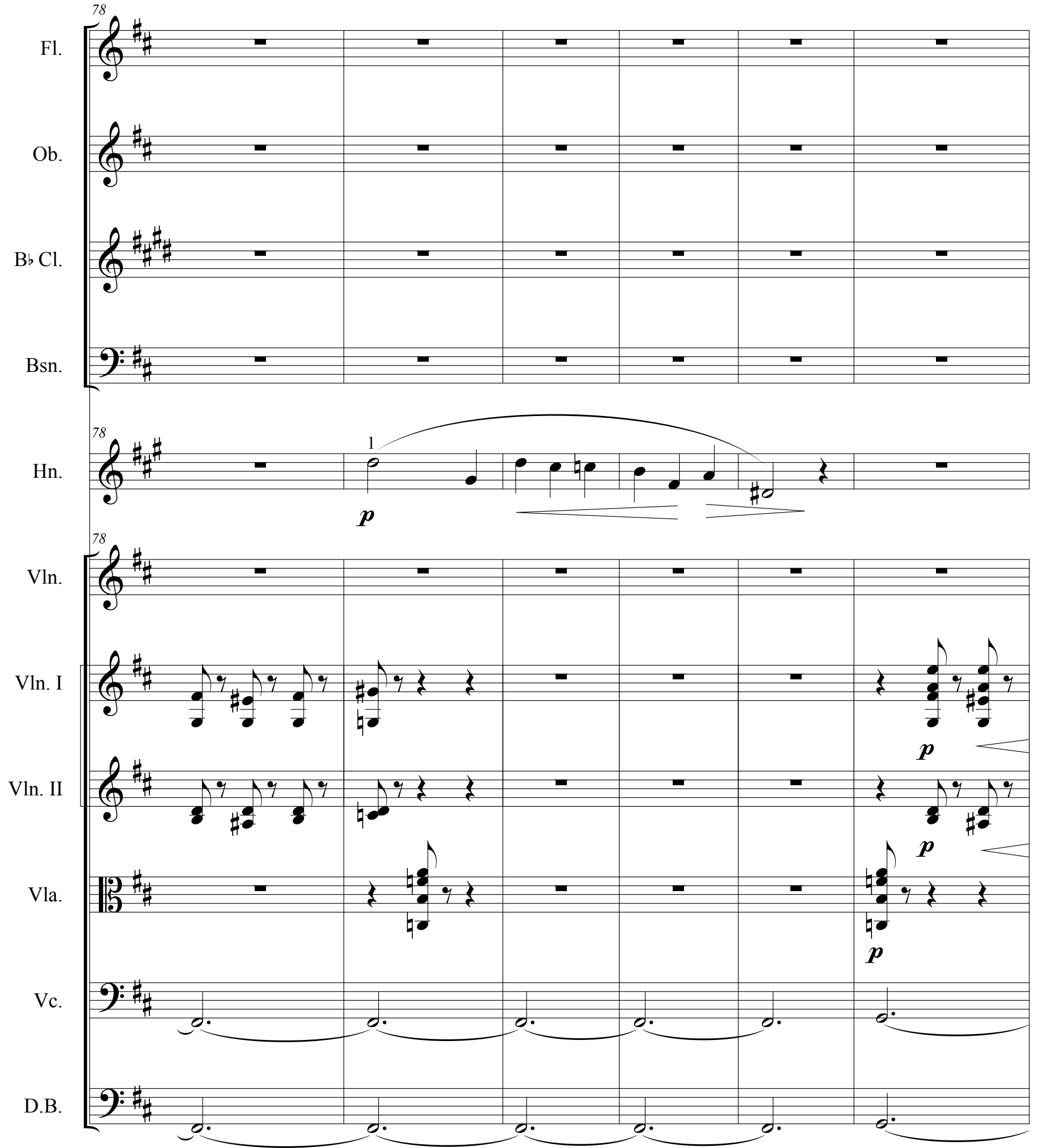


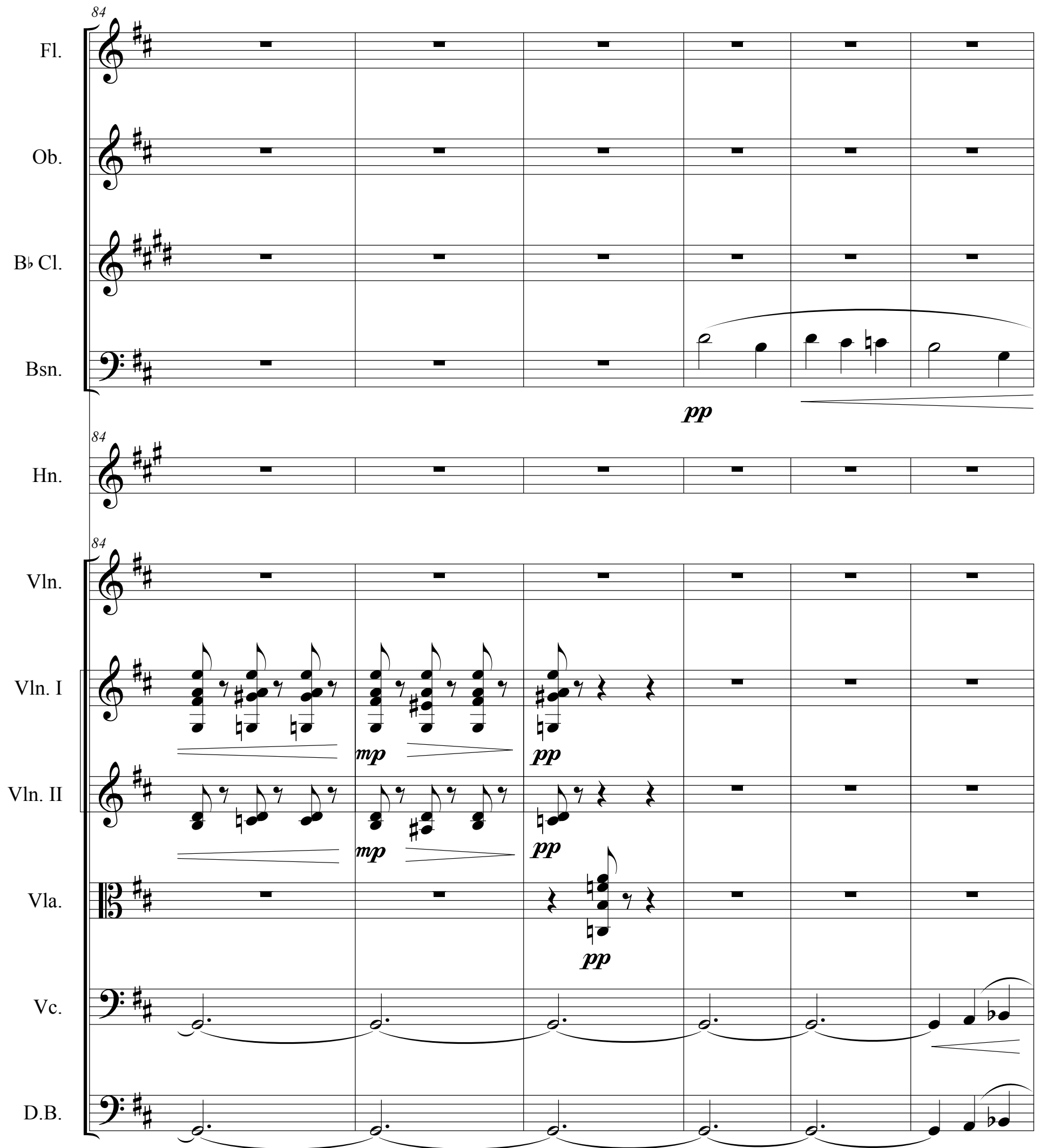




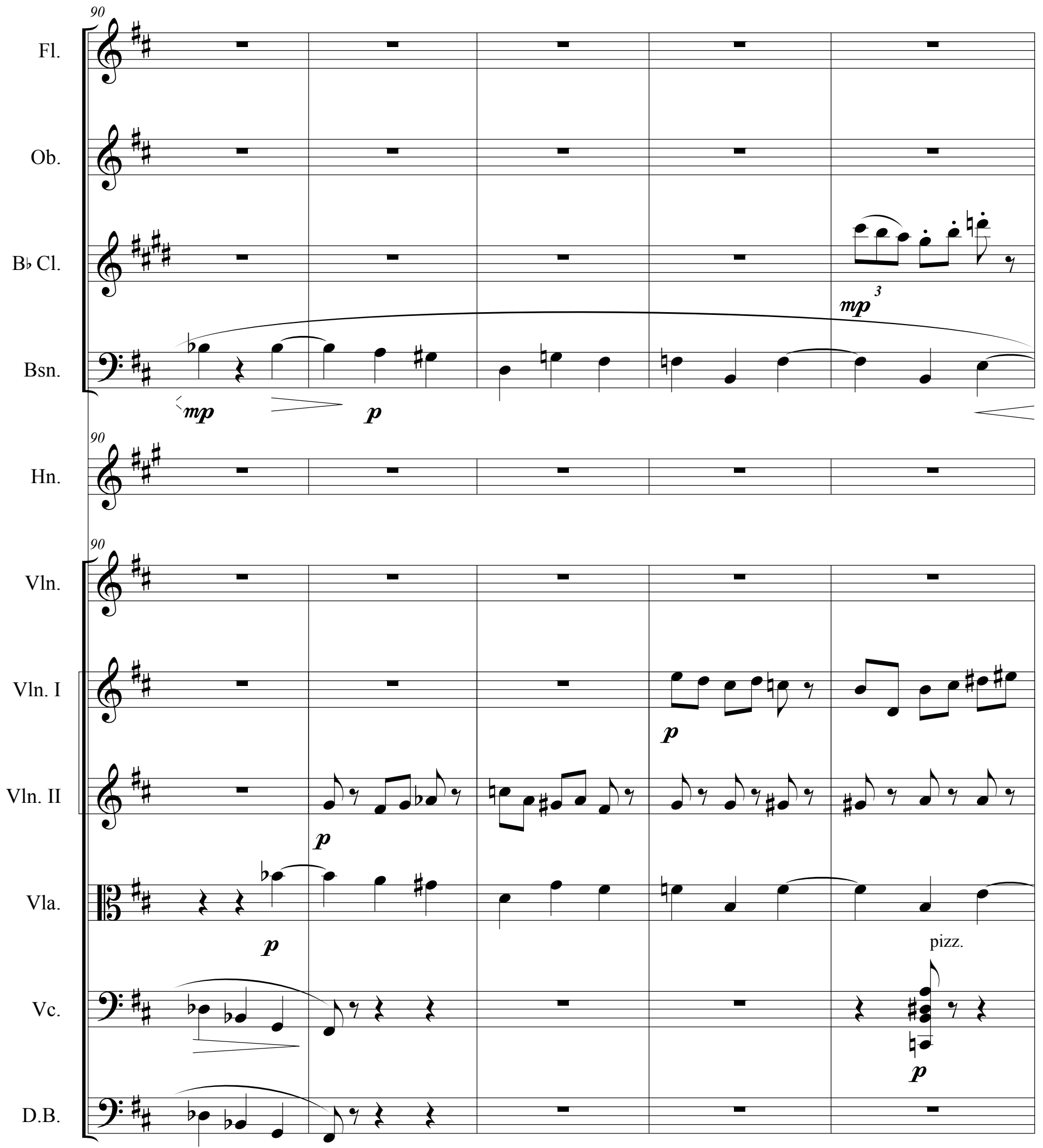




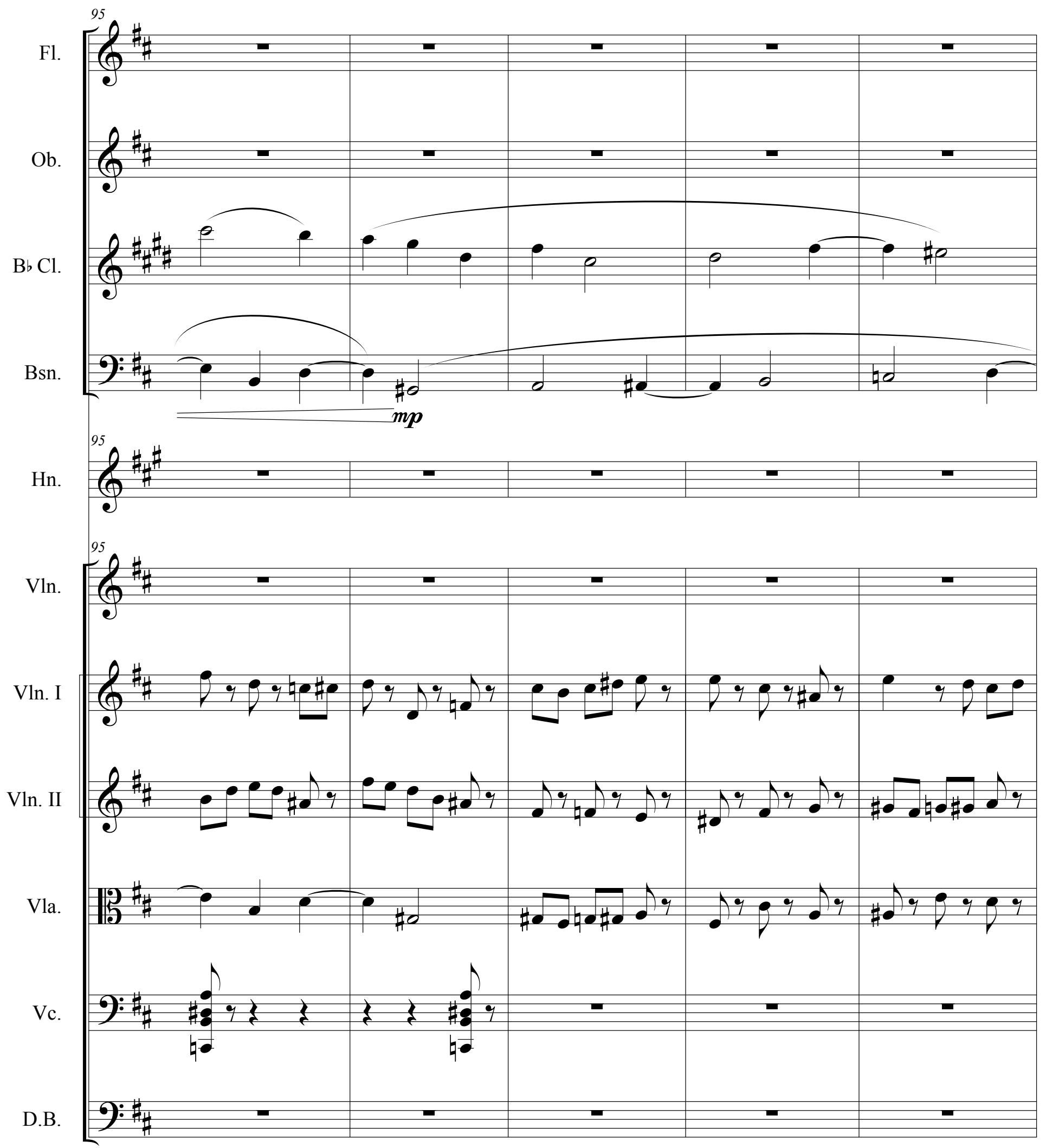



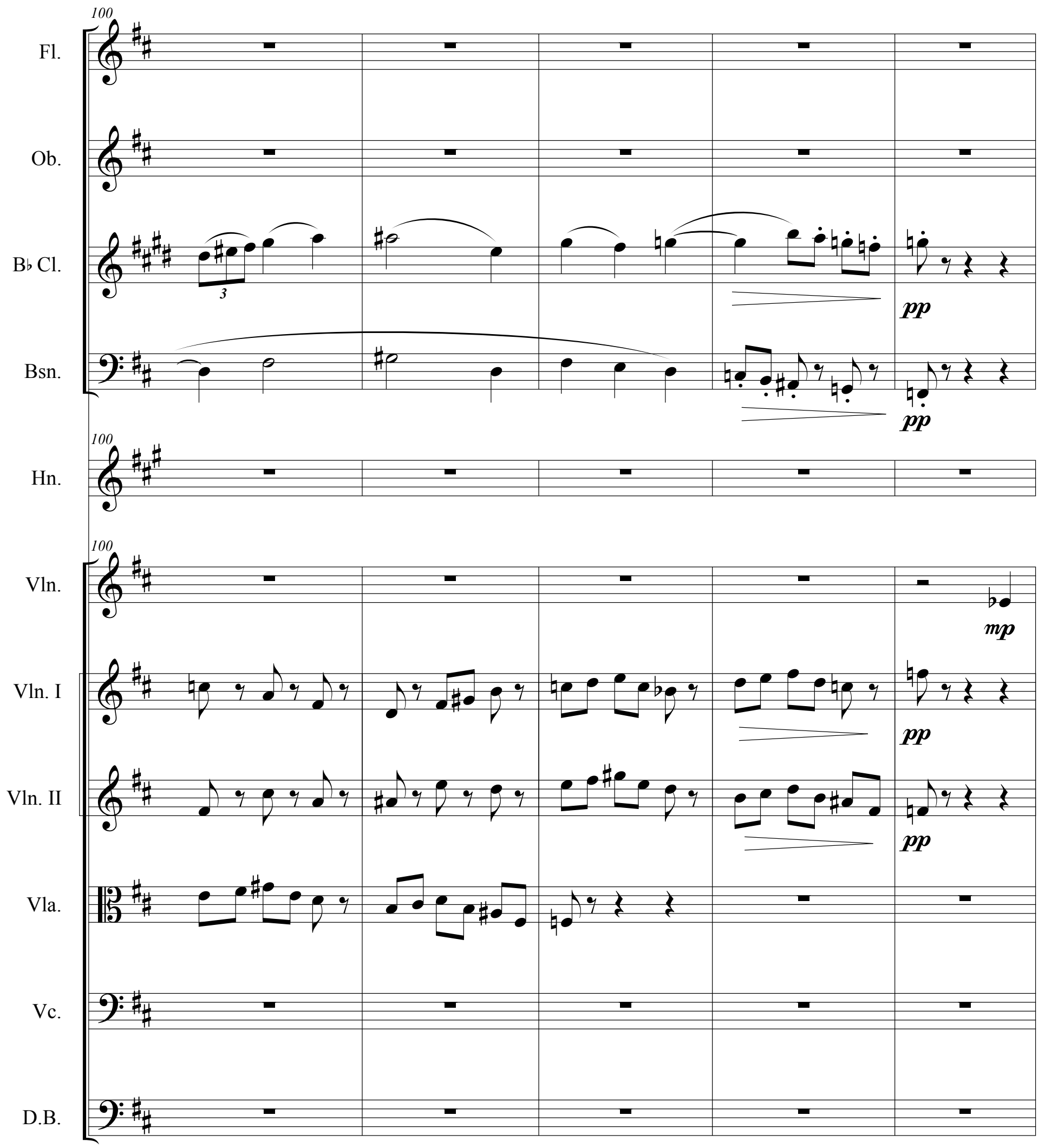
Score

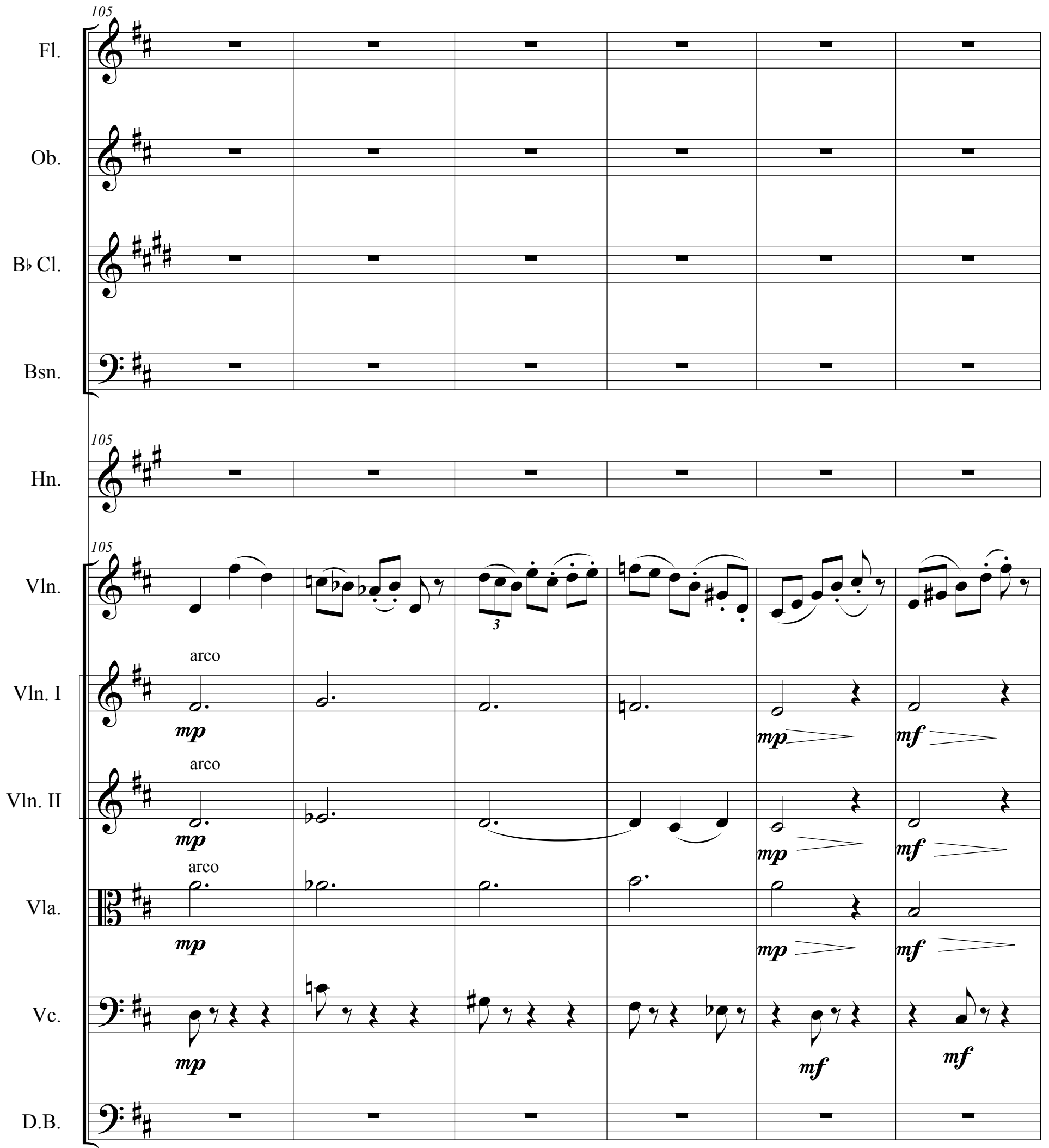



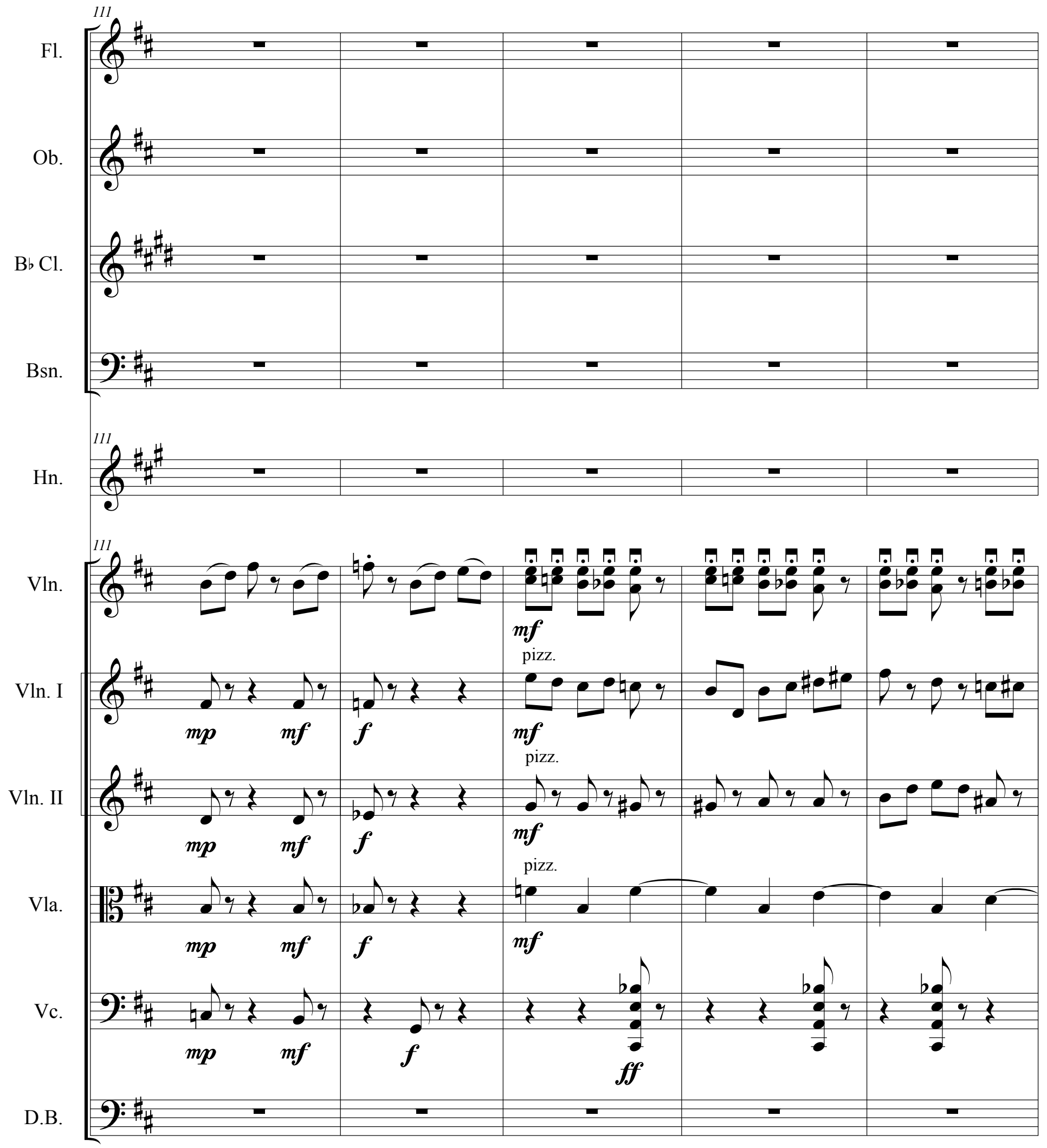
Score

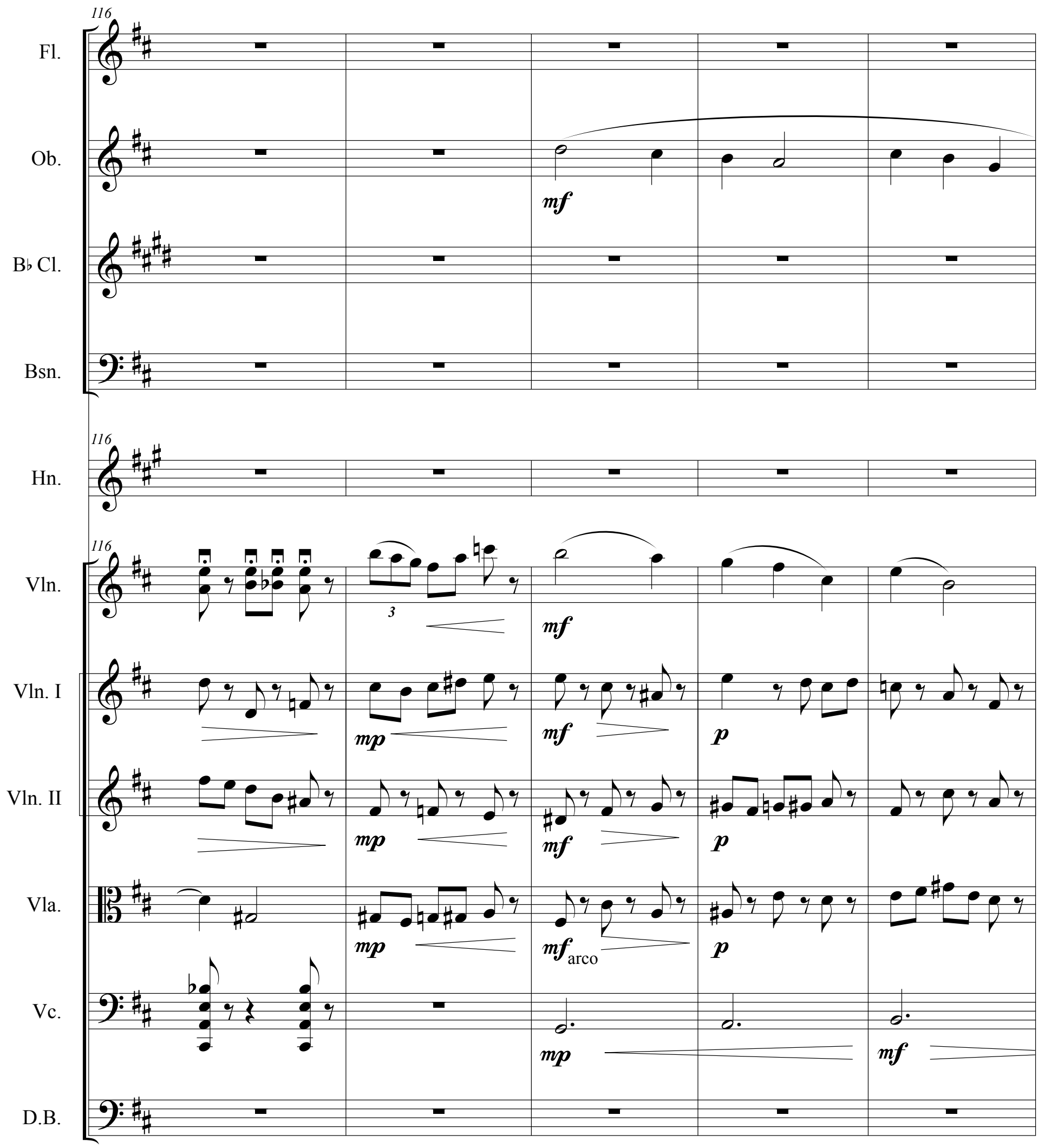


34

For Violin Solo and Chamber Orchestra

Fl.

Ob.

$\mathrm{B} b \mathrm{Cl}$.

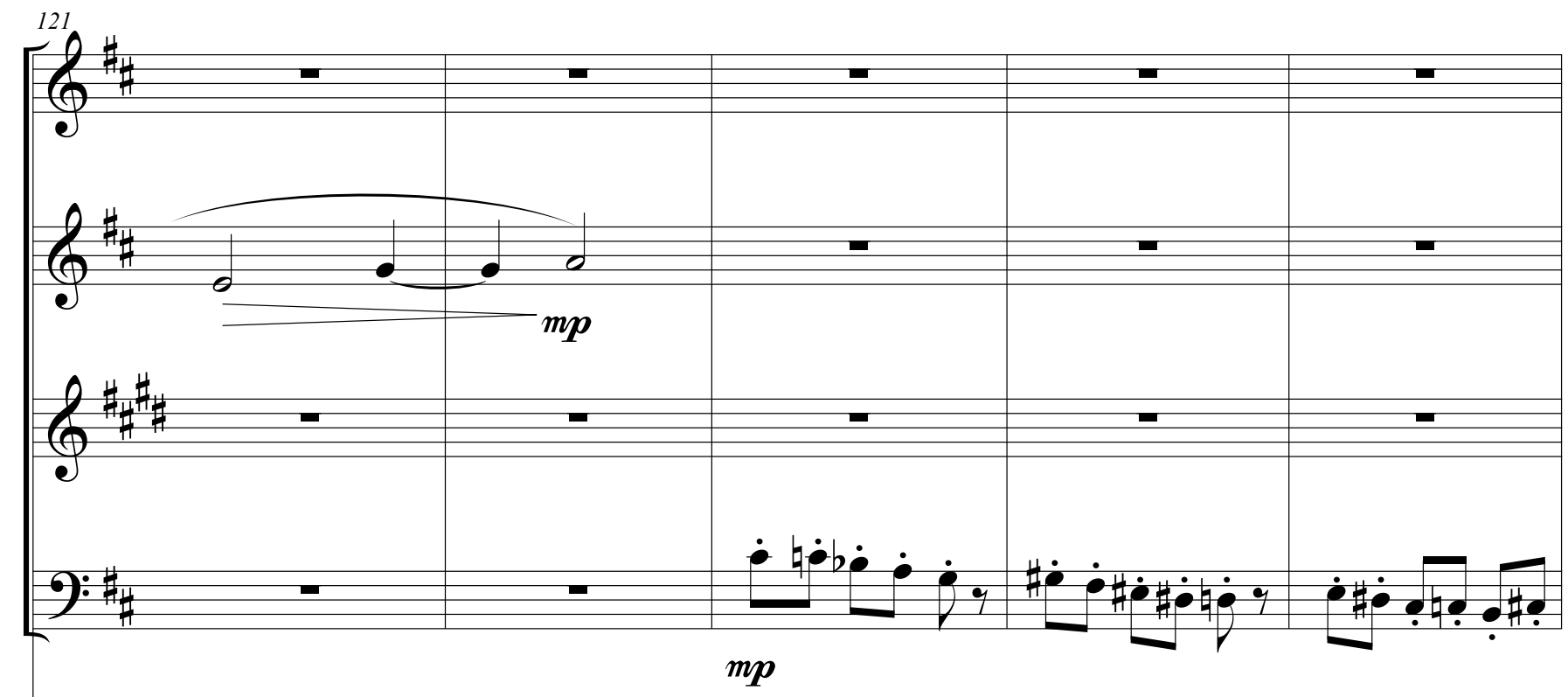

Hon.

$\int_{0}^{121}$

Vln.

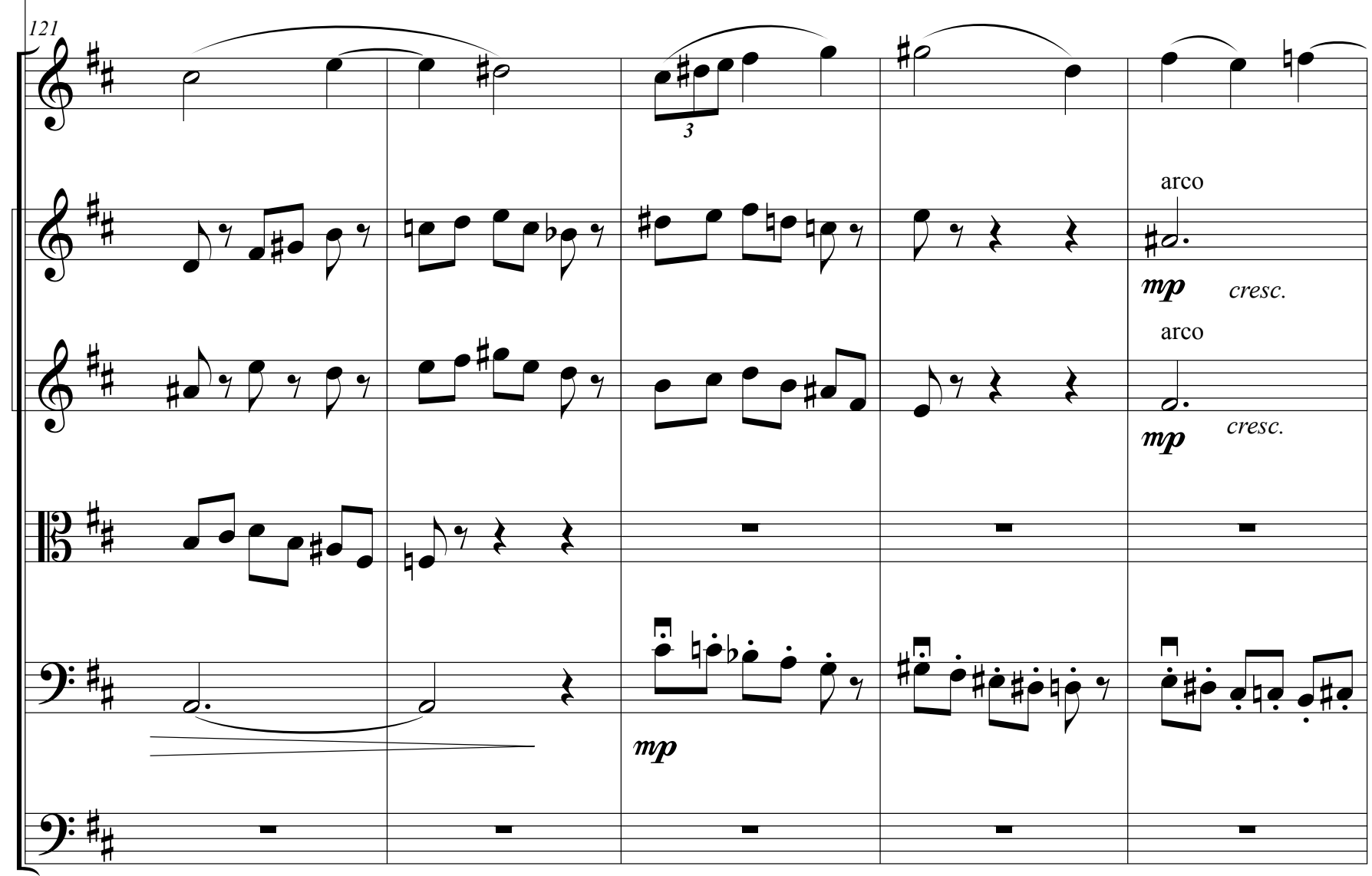

Ven. I

Vln. II

VIa.

Vc.

D.B. 
Score

For Violin Solo and Chamber Orchestra

35

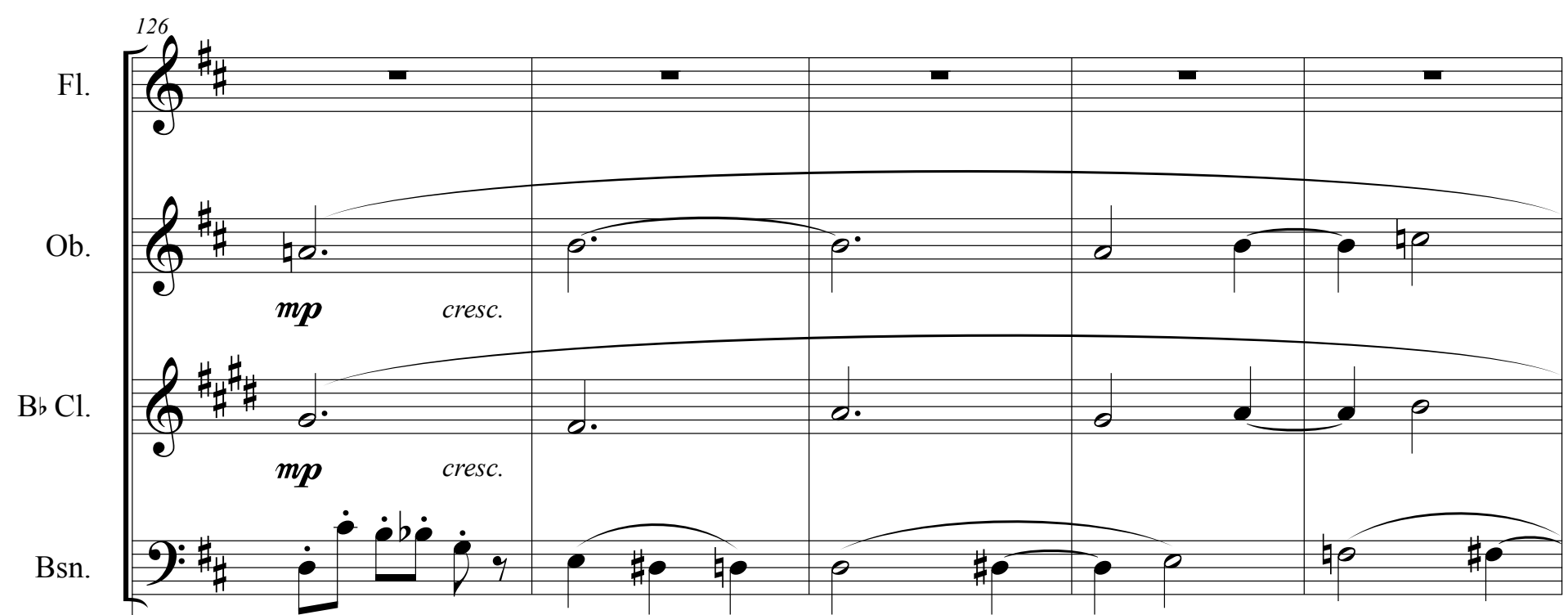

Vln. I

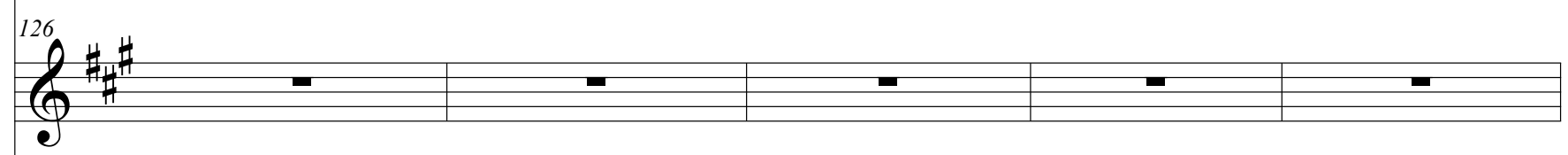

Vln.

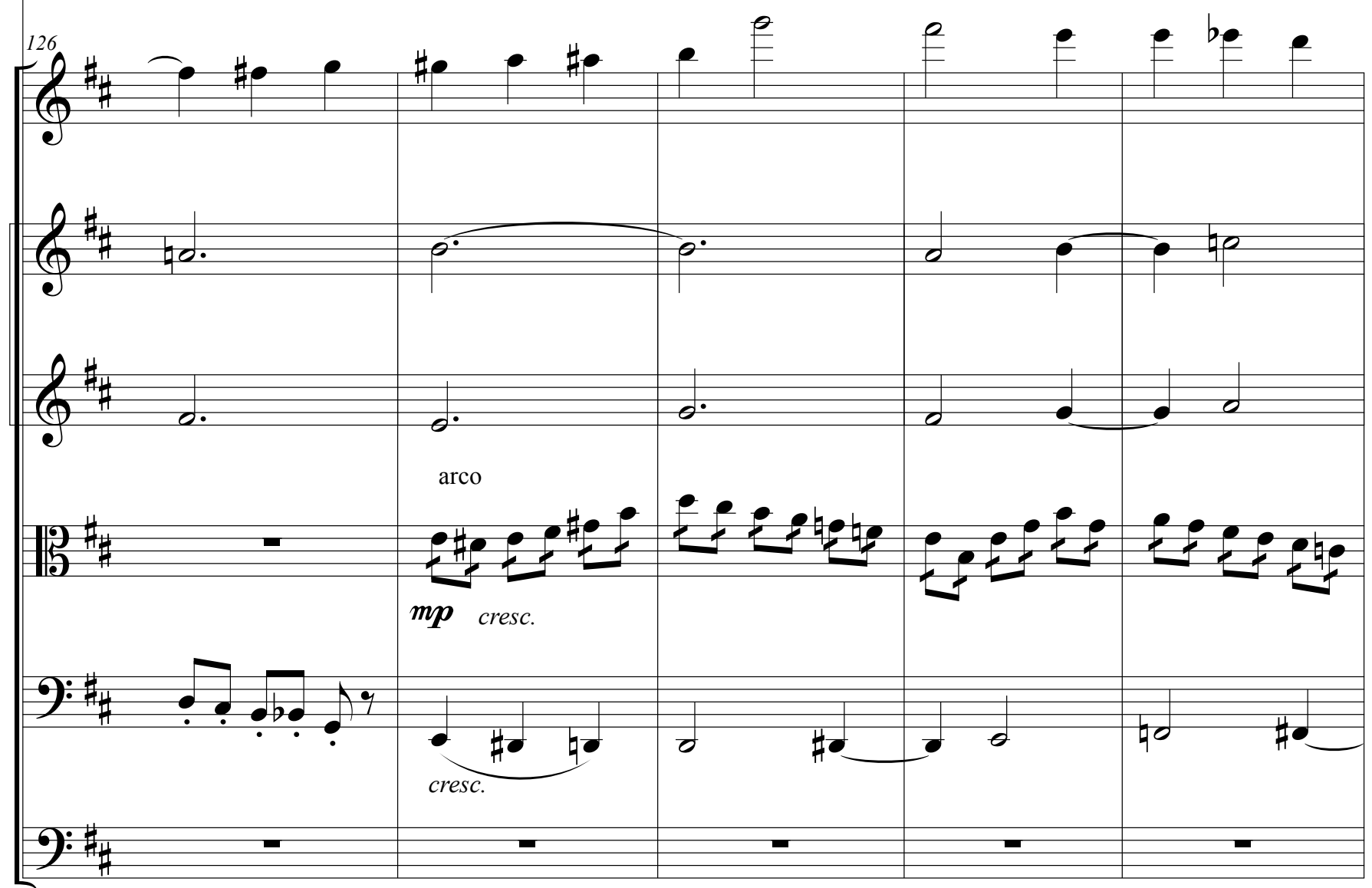



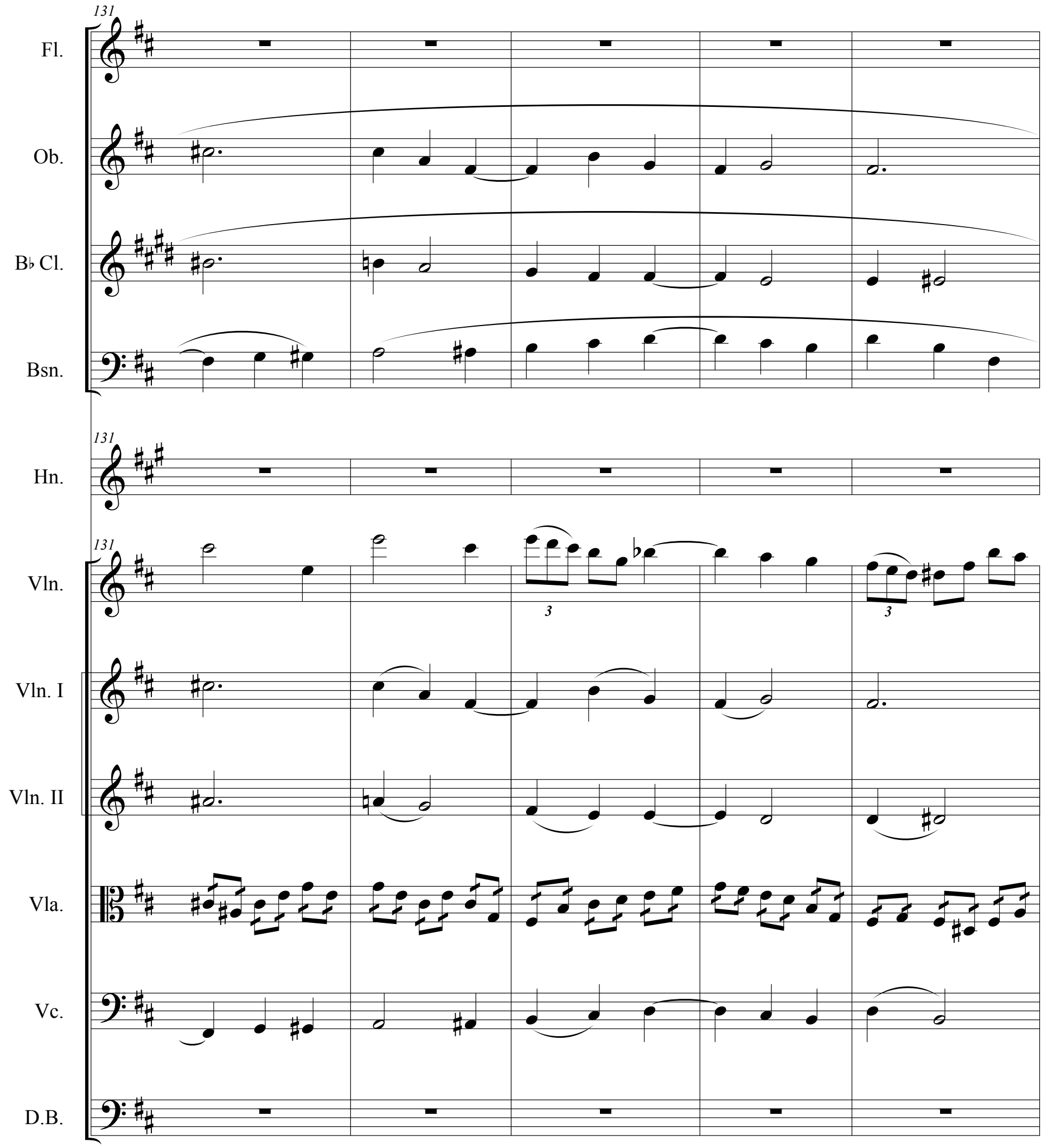
Score

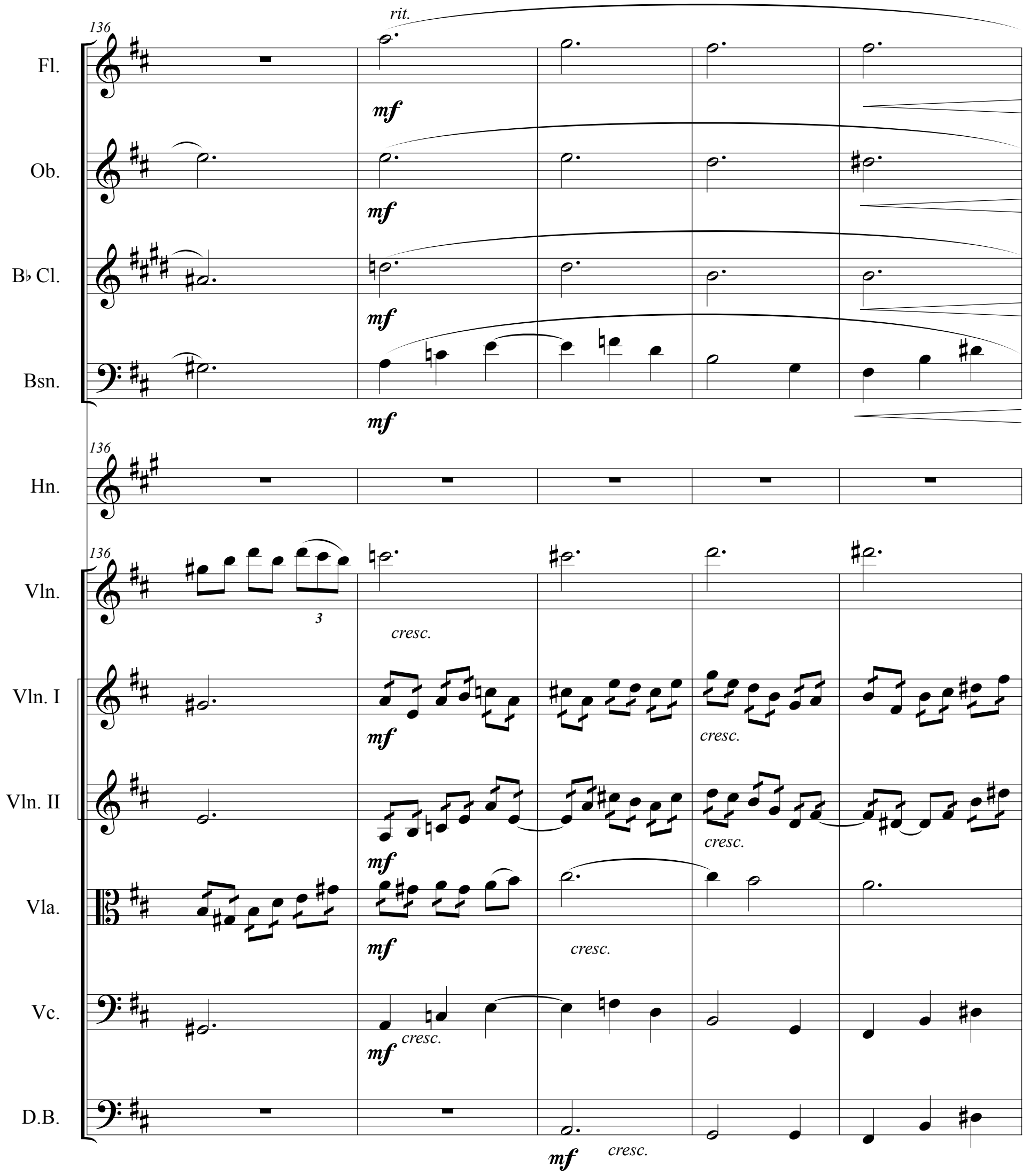


Maestoso

Fl.

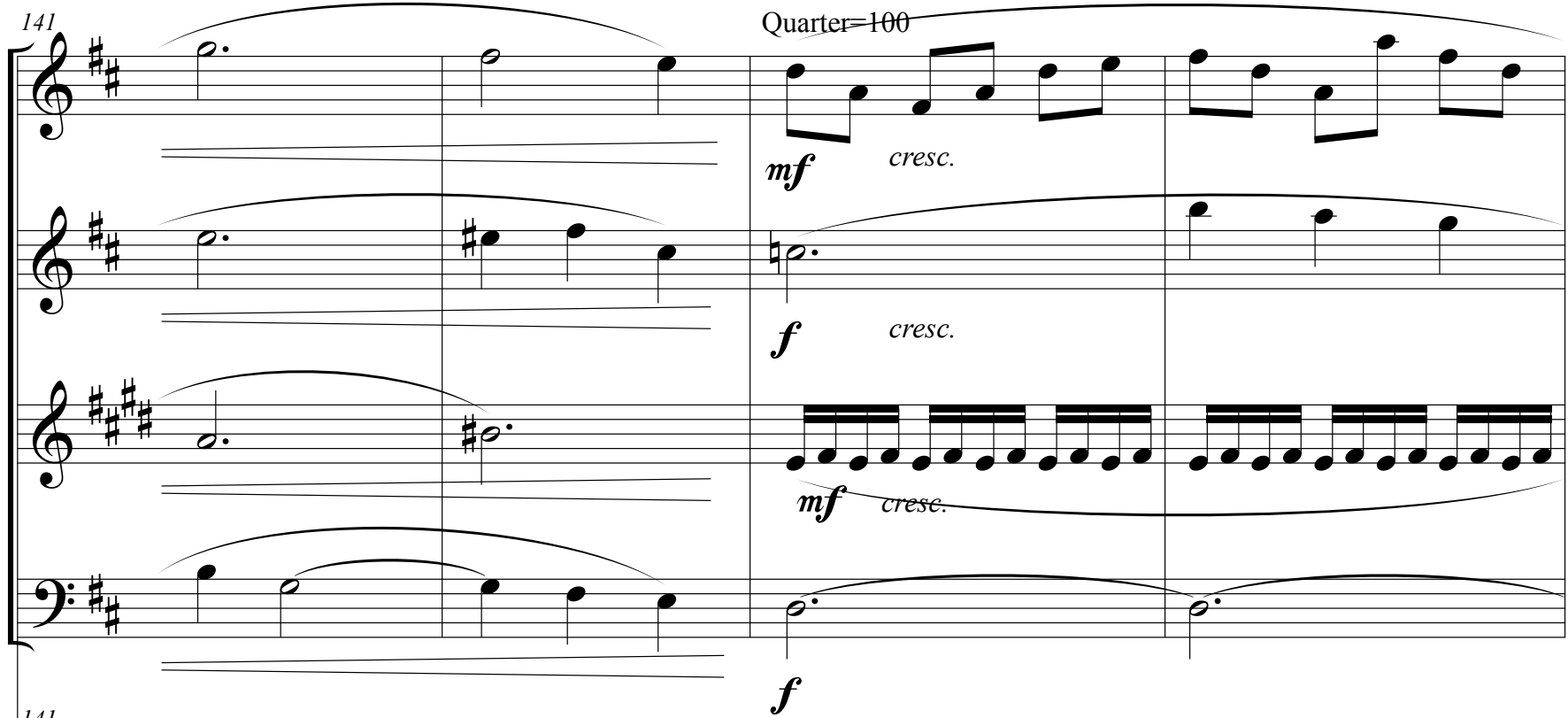

$\mathrm{B} b \mathrm{Cl}$.

Bsn.

$\mathrm{Ob}$.

Hn.

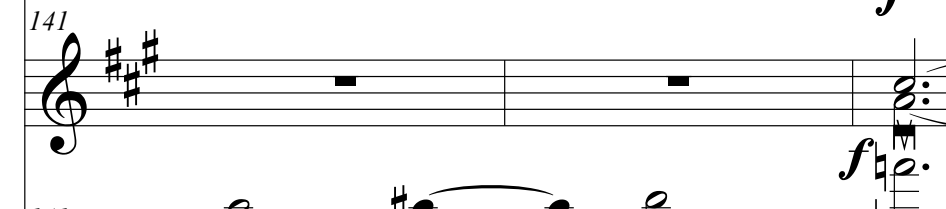

Vln.

Vln. I

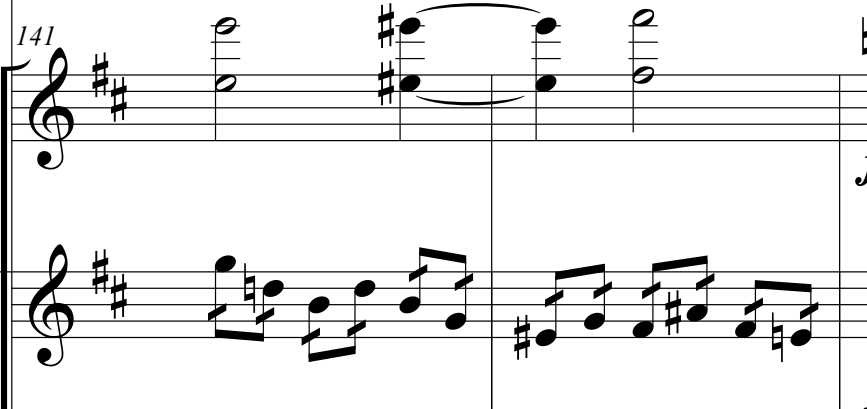

$\bar{e}^{\circ}$

ff

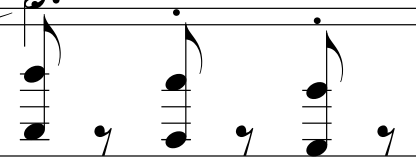

Vln. II

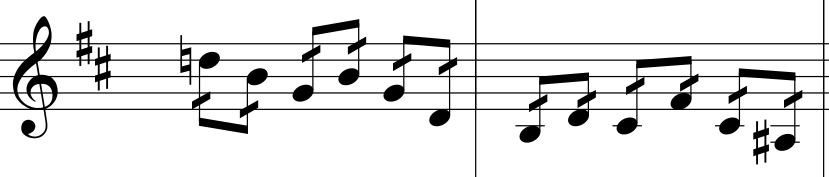

Vla.

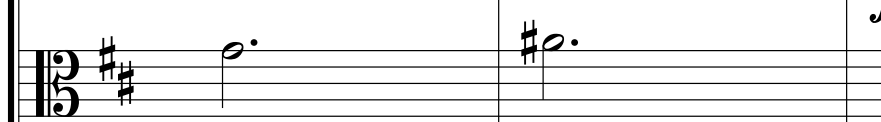

Vc.

D.B.

$9:$

9:

$+\frac{1}{\hbar-2}$
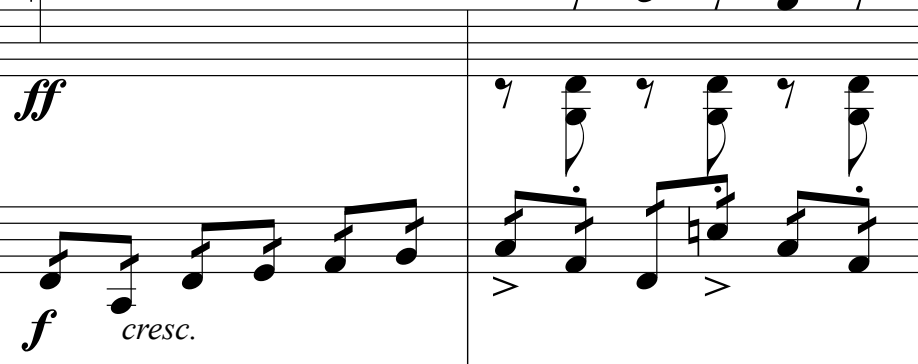

$\frac{2}{20}$
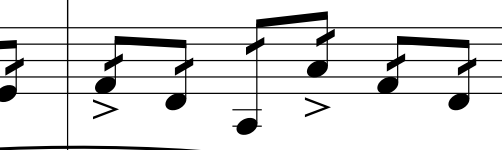

f
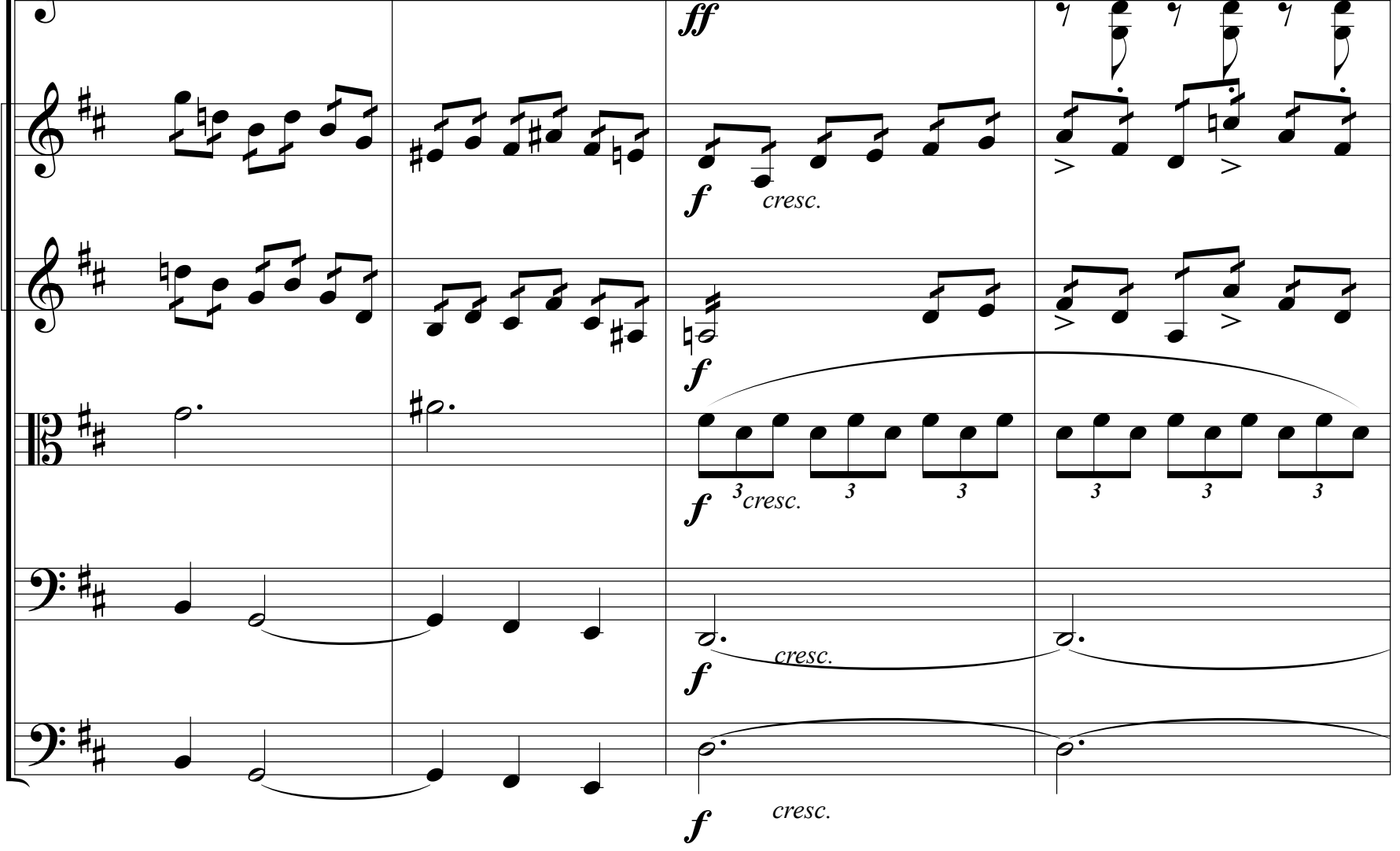
Fl.

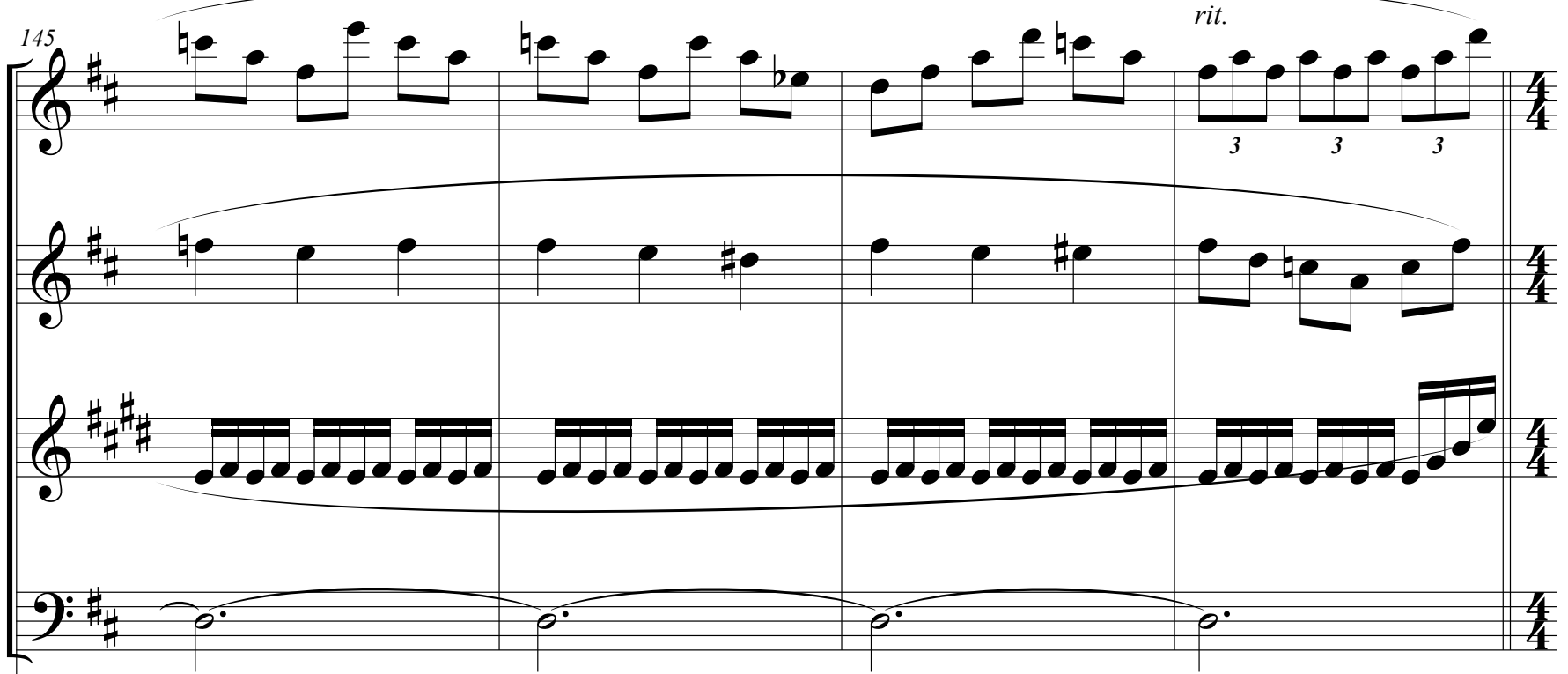

Bsn.

$\mathrm{Ob}$.

$\mathrm{B} b \mathrm{Cl}$.

Hn.

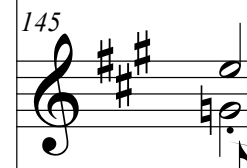

Vln.

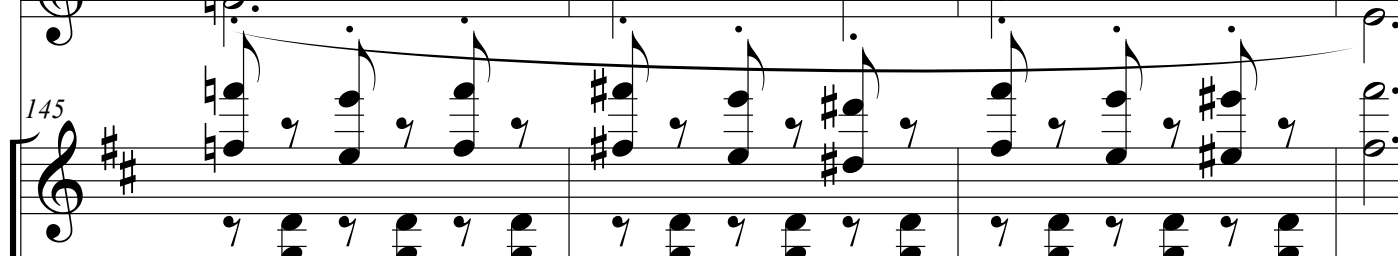

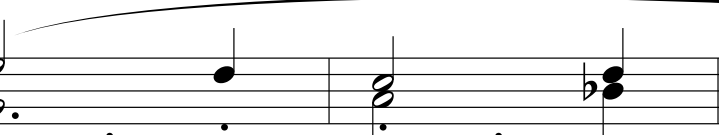
8:

Vln. I

Vln. II

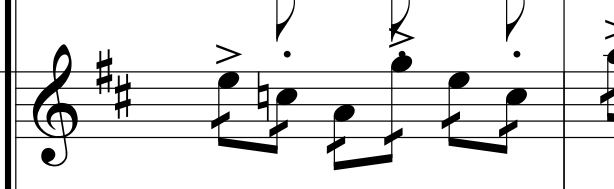

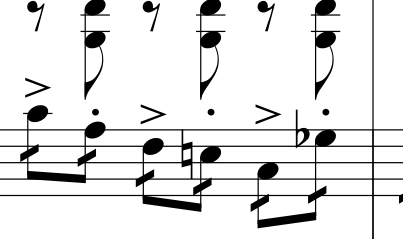

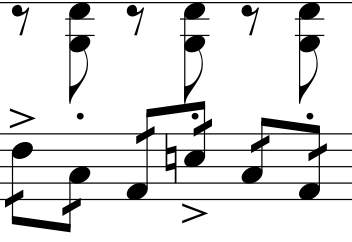

2

4

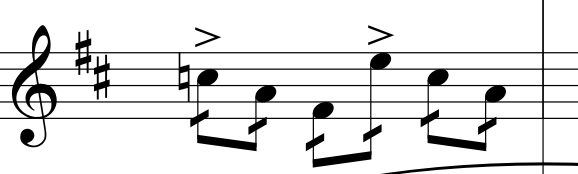
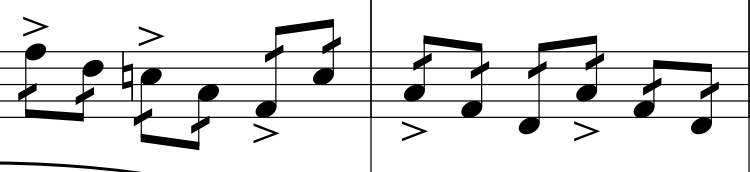

$5=4$

Vla.

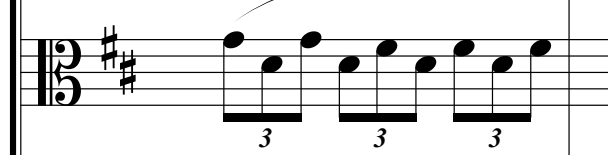
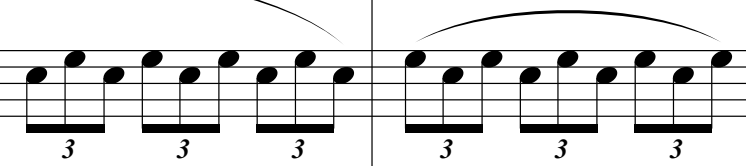

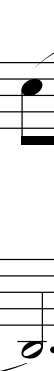

D.B.

\%:

0

o.

$9: \pi \quad \rho^{\circ}$ 
Score

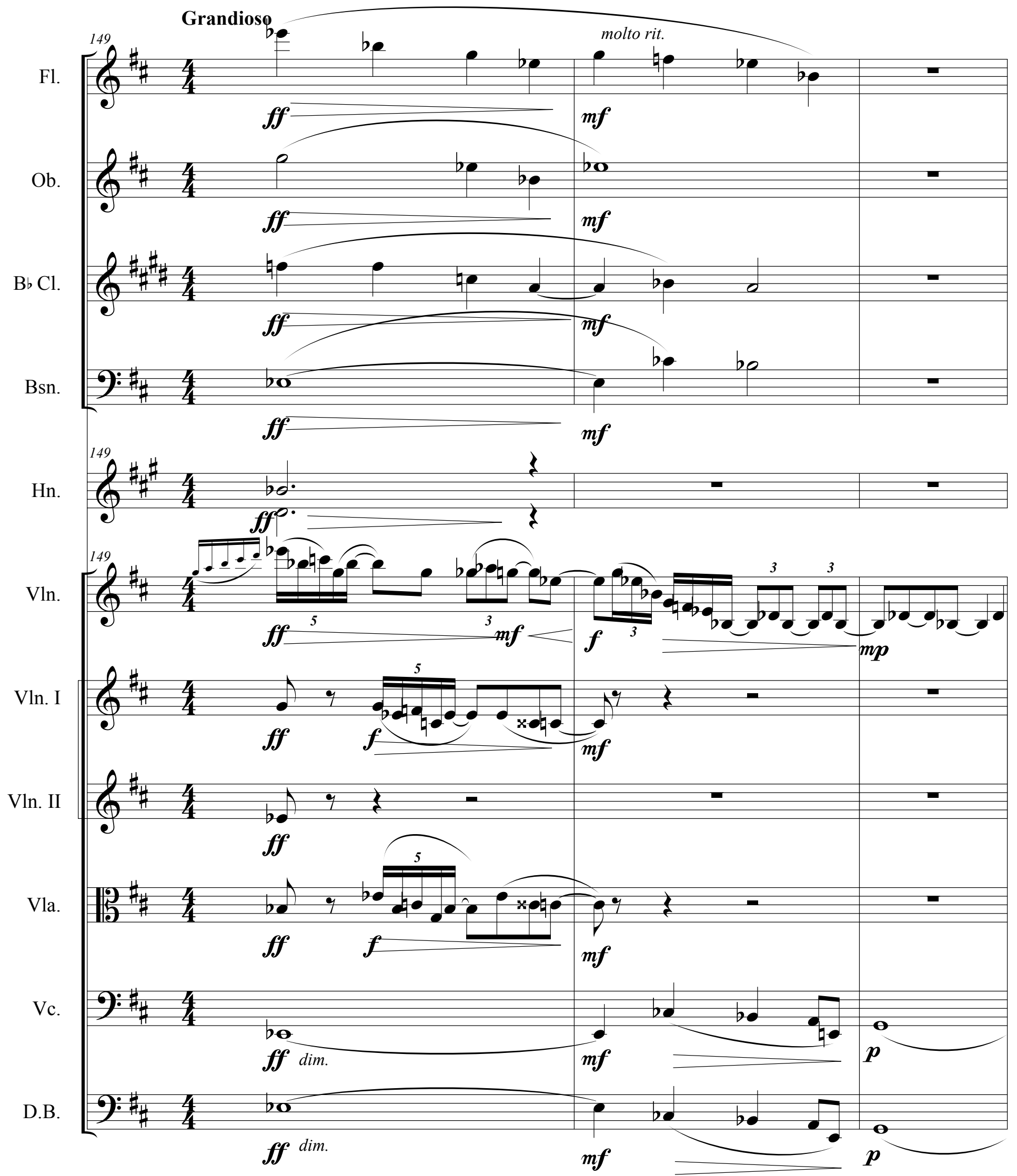


Score

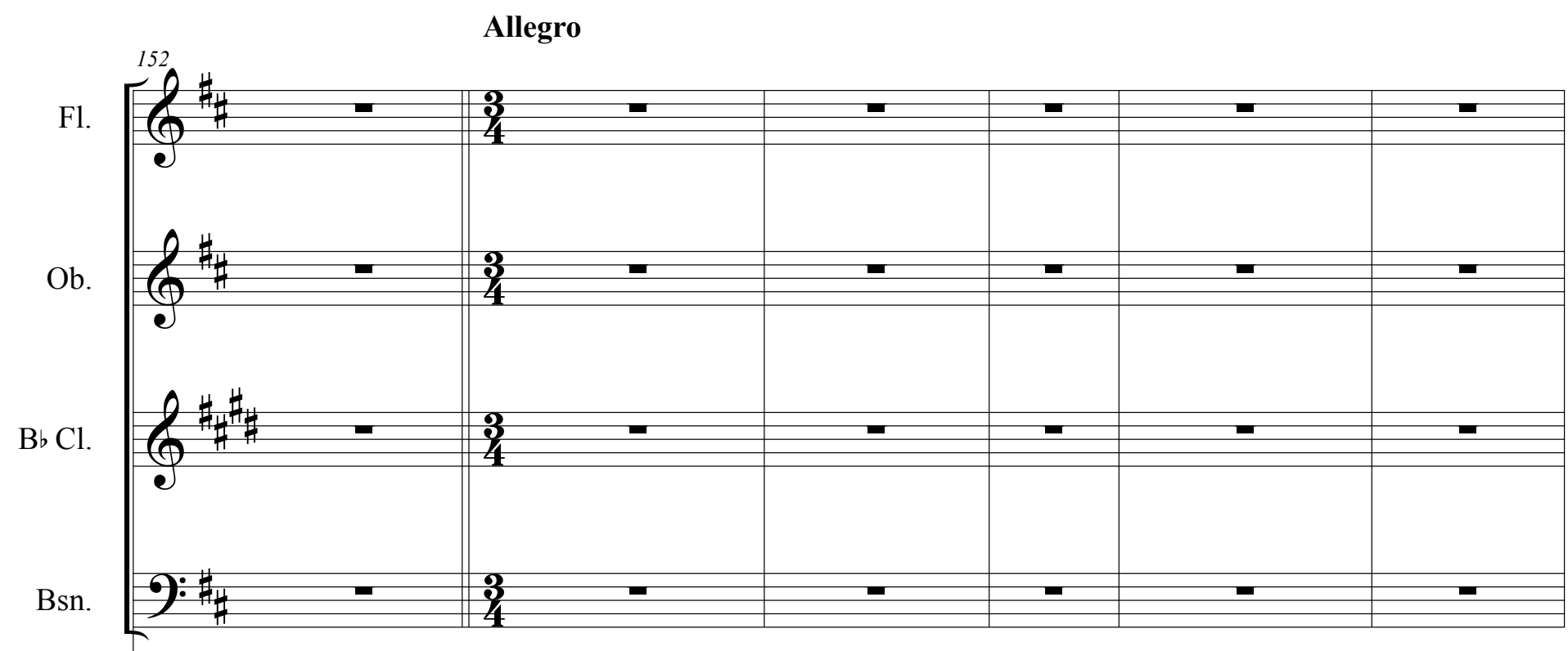

Hn.
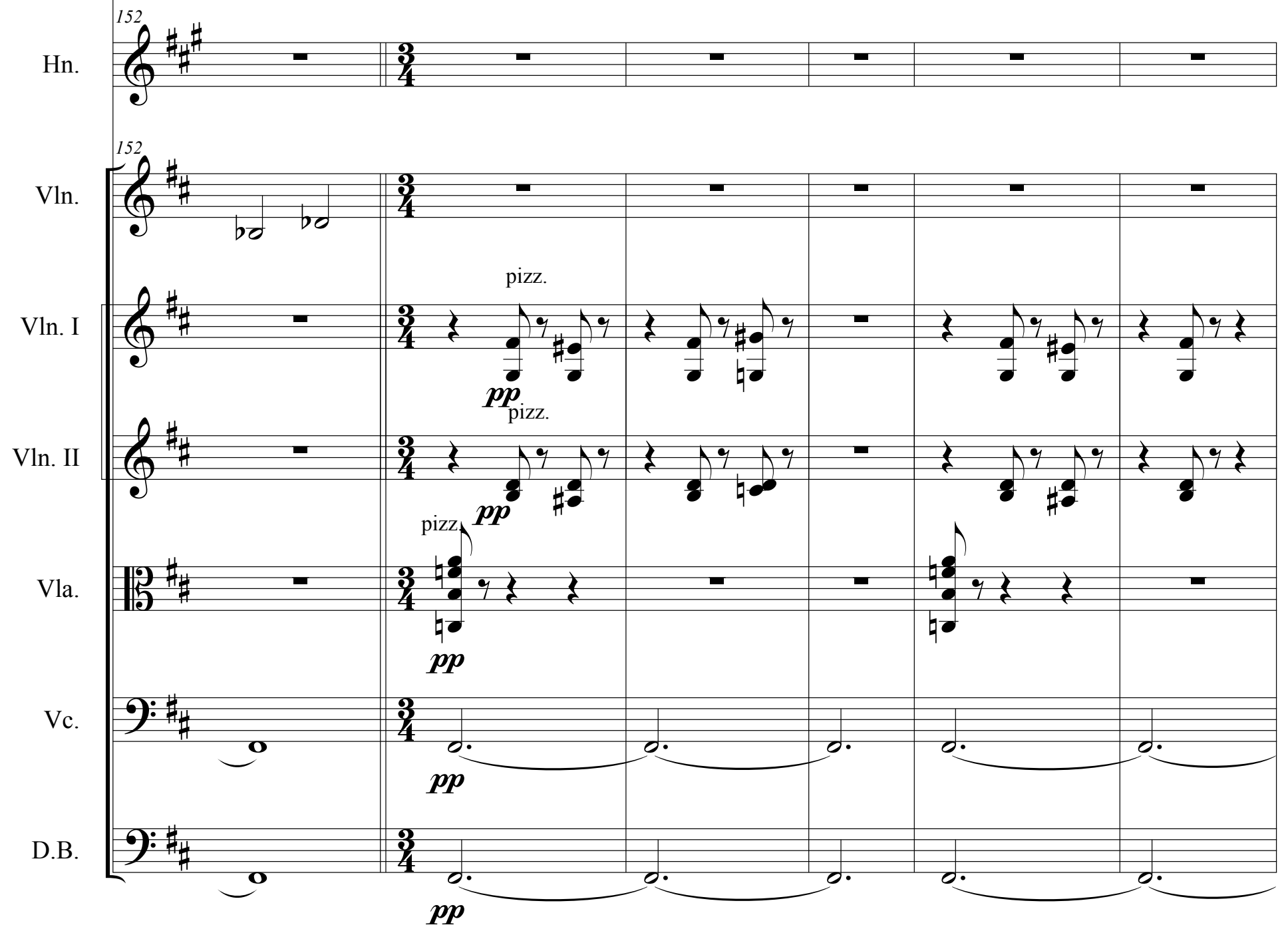


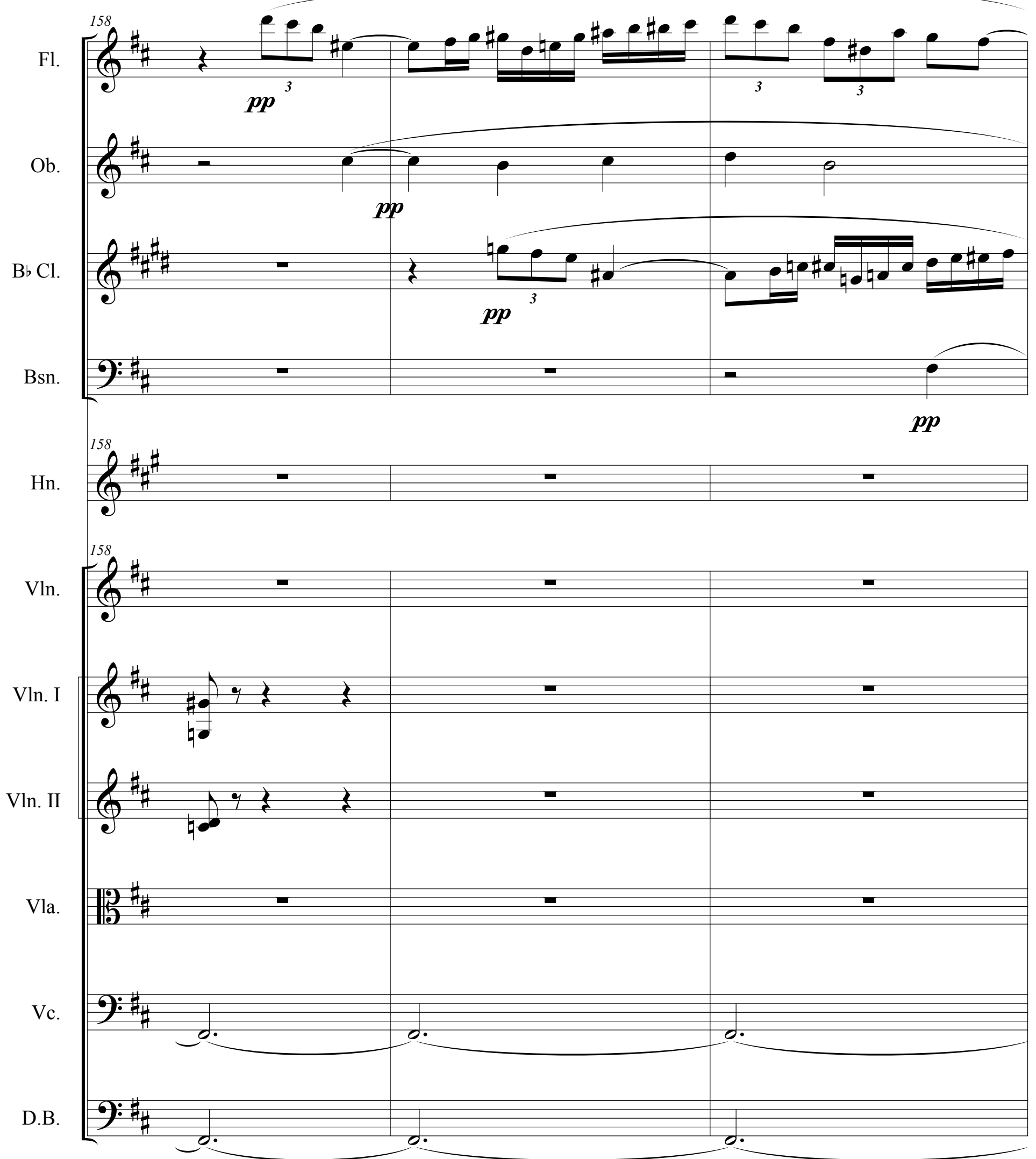


Score

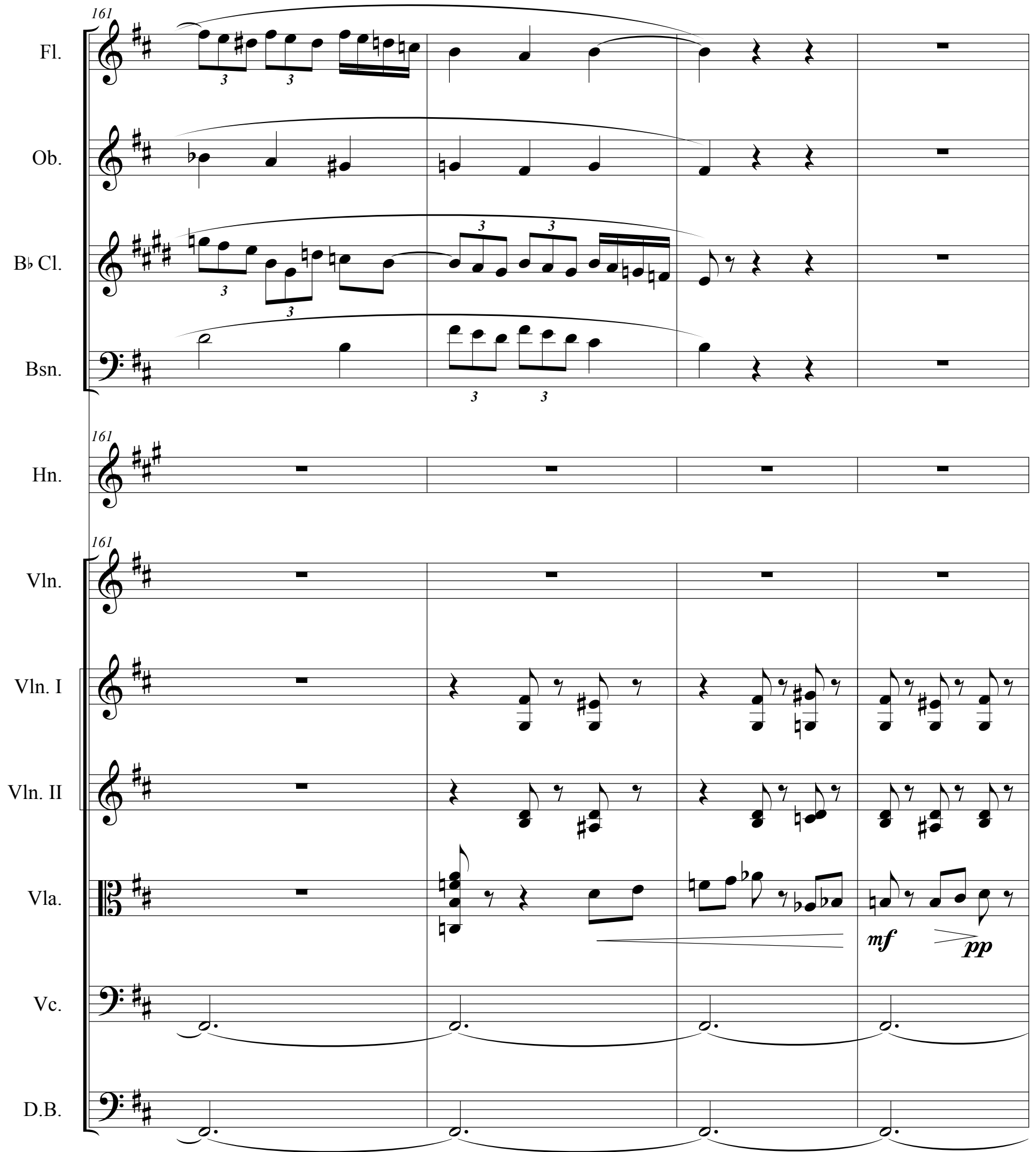


44

For Violin Solo and Chamber Orchestra

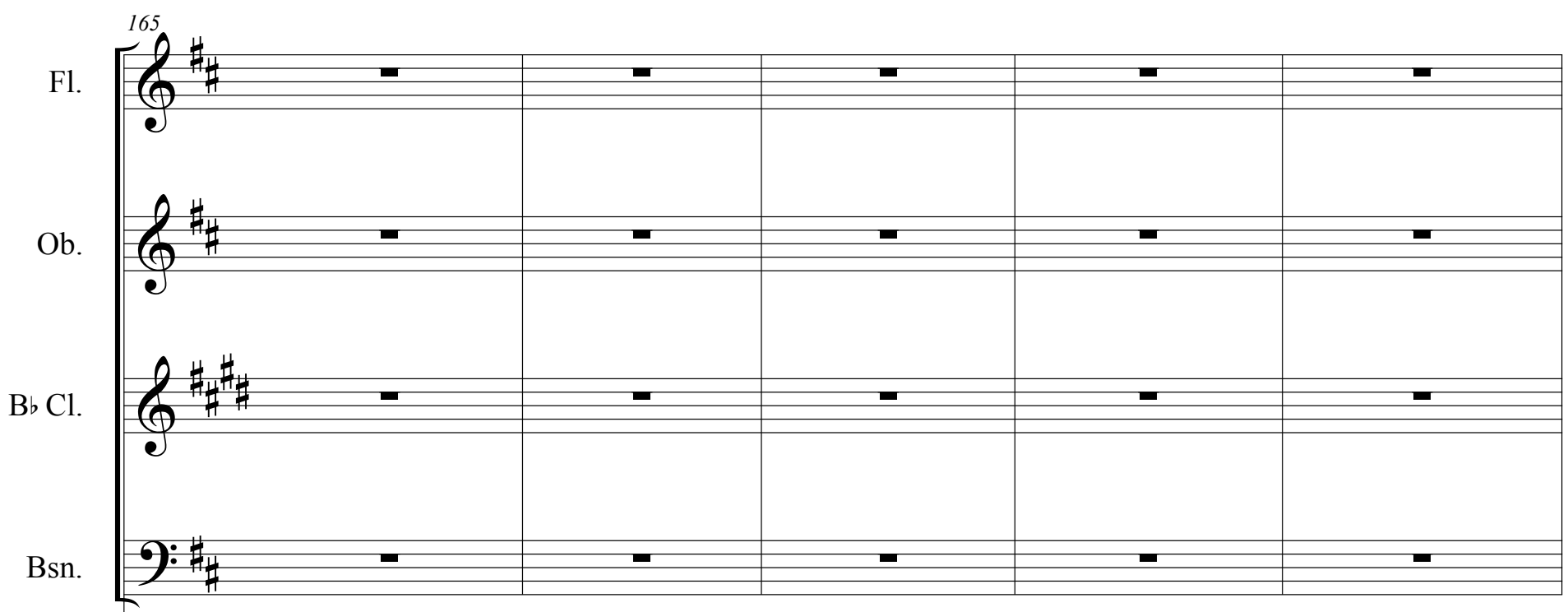

Vln. I

Vln.

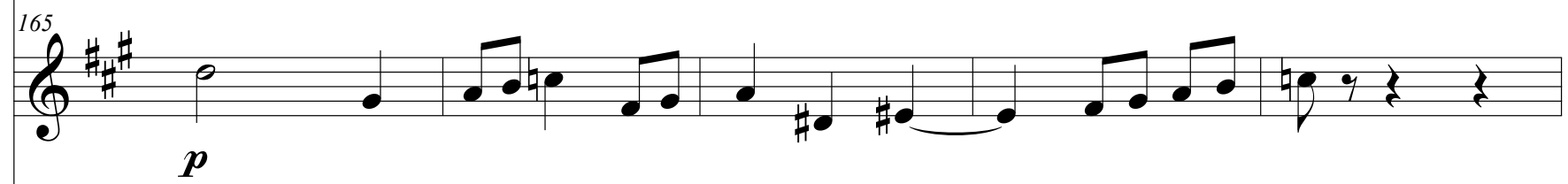

Vln. II

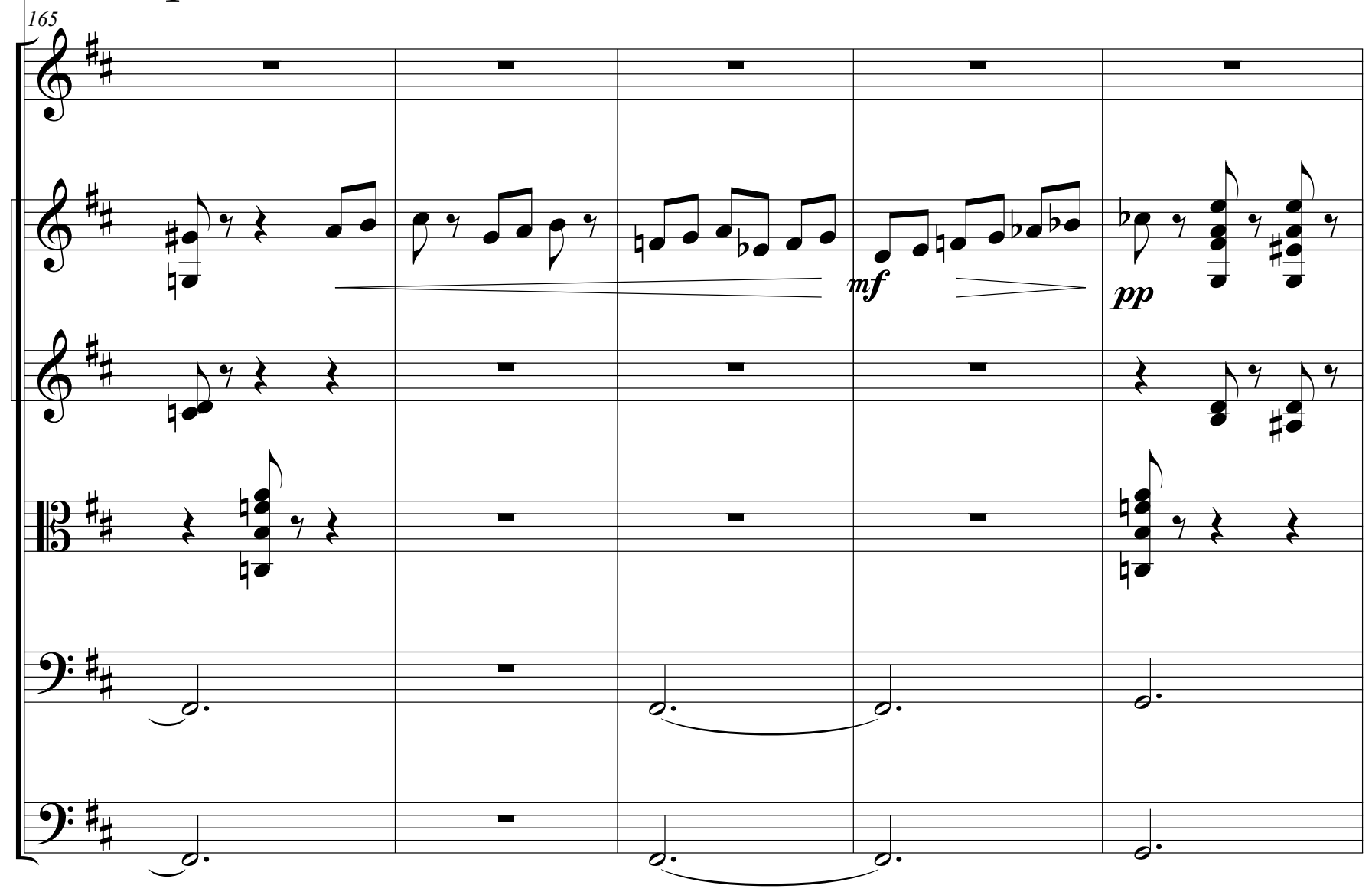


Score

For Violin Solo and Chamber Orchestra

45

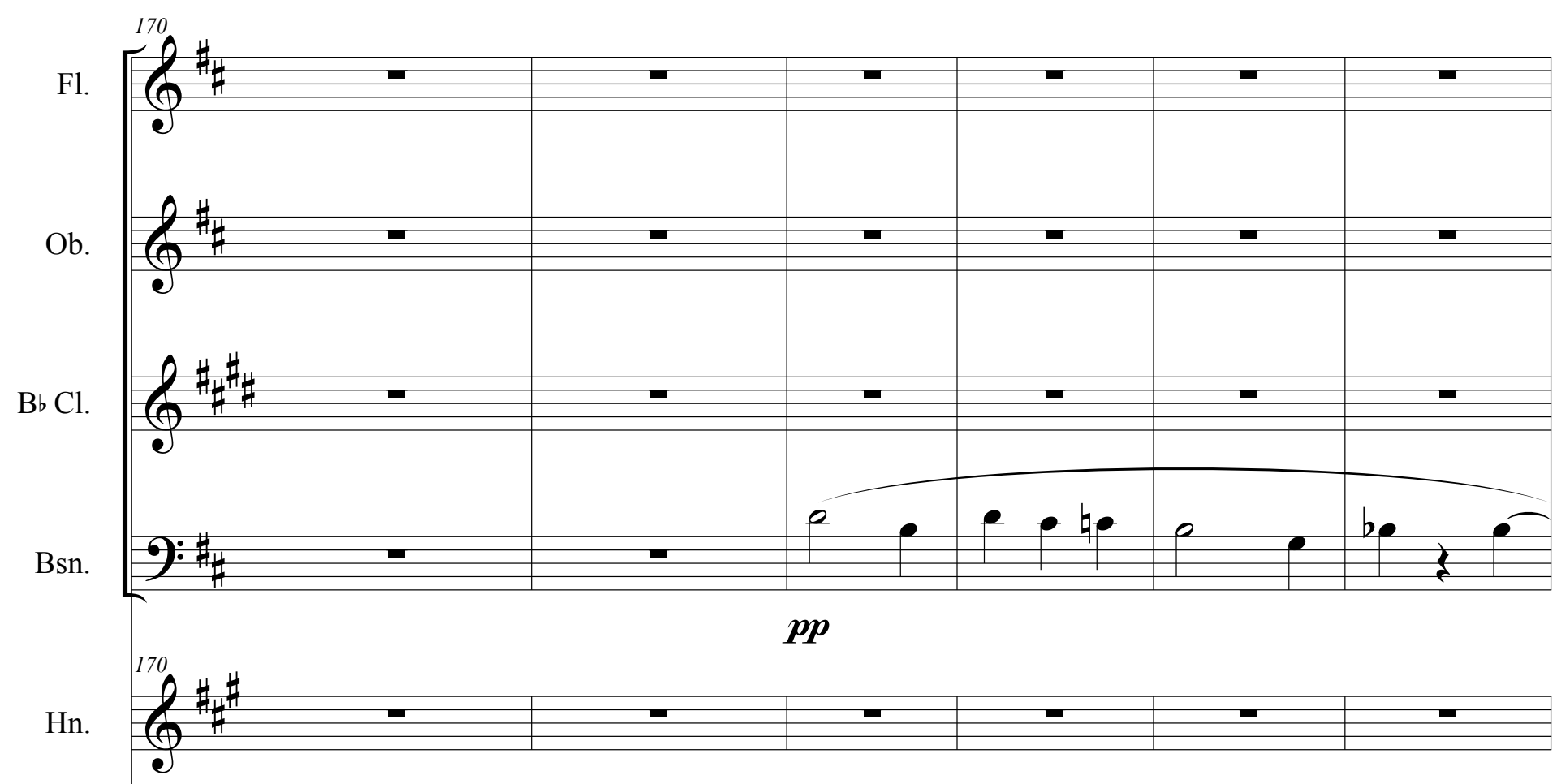

Vln. I

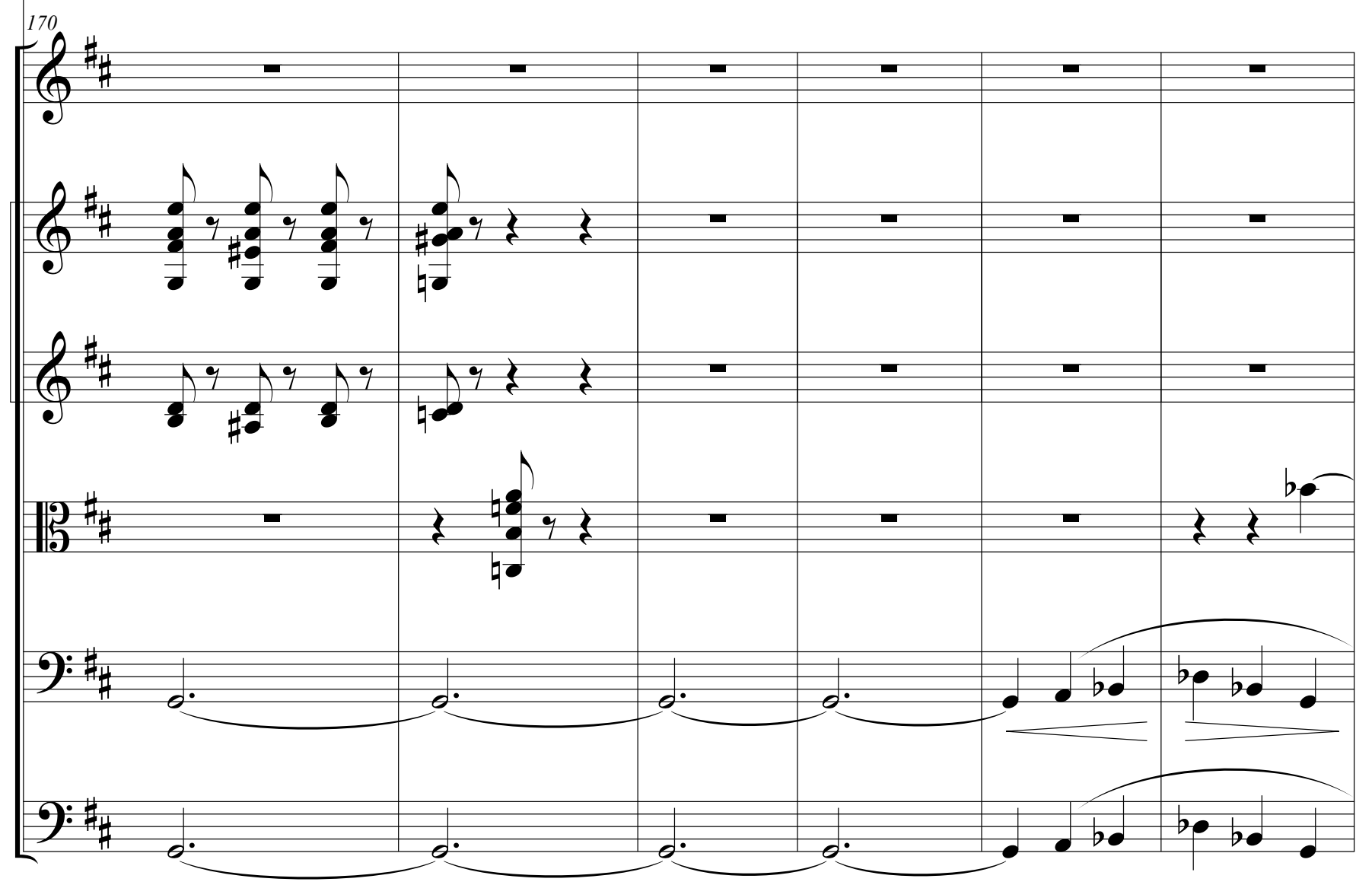



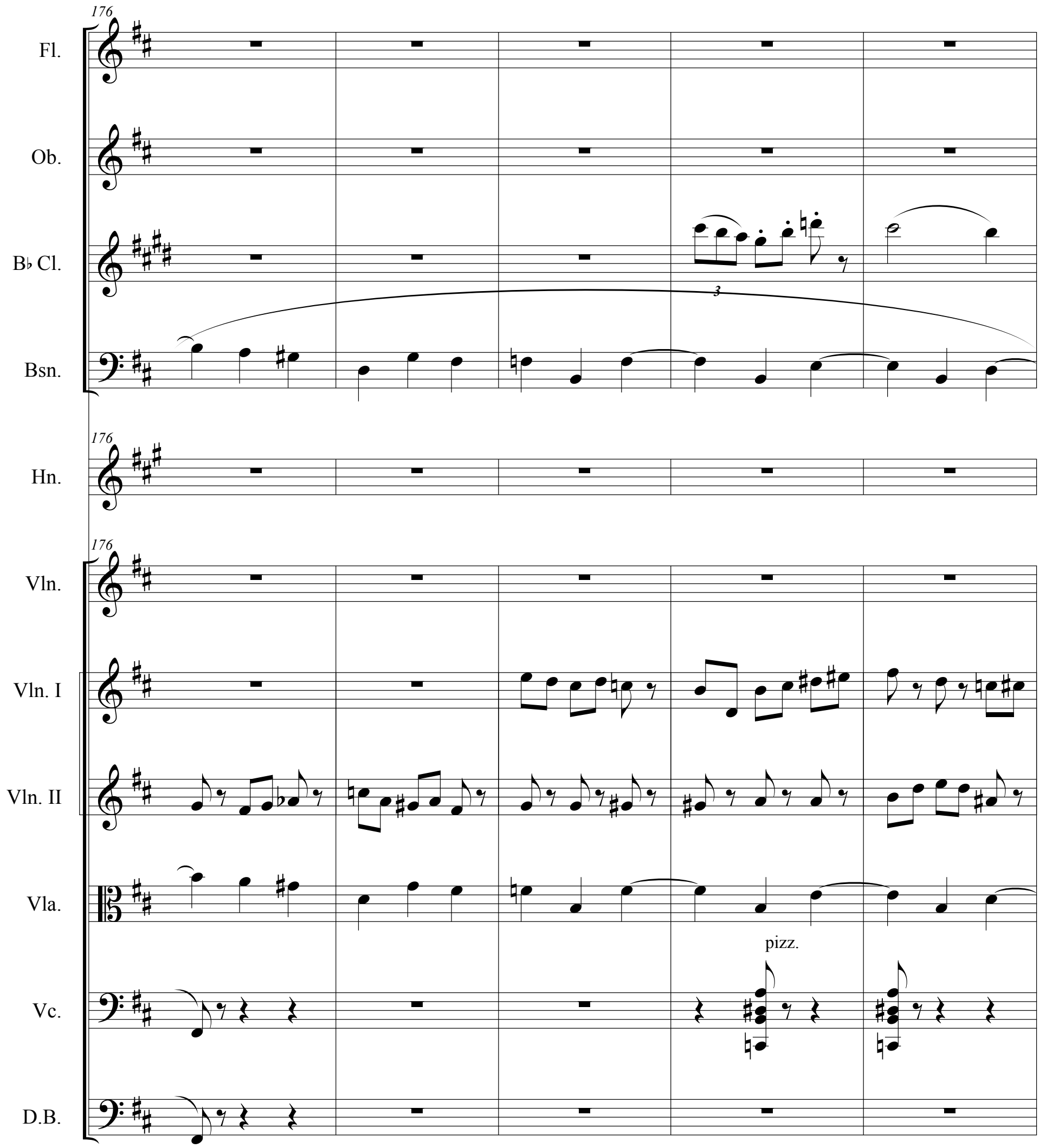

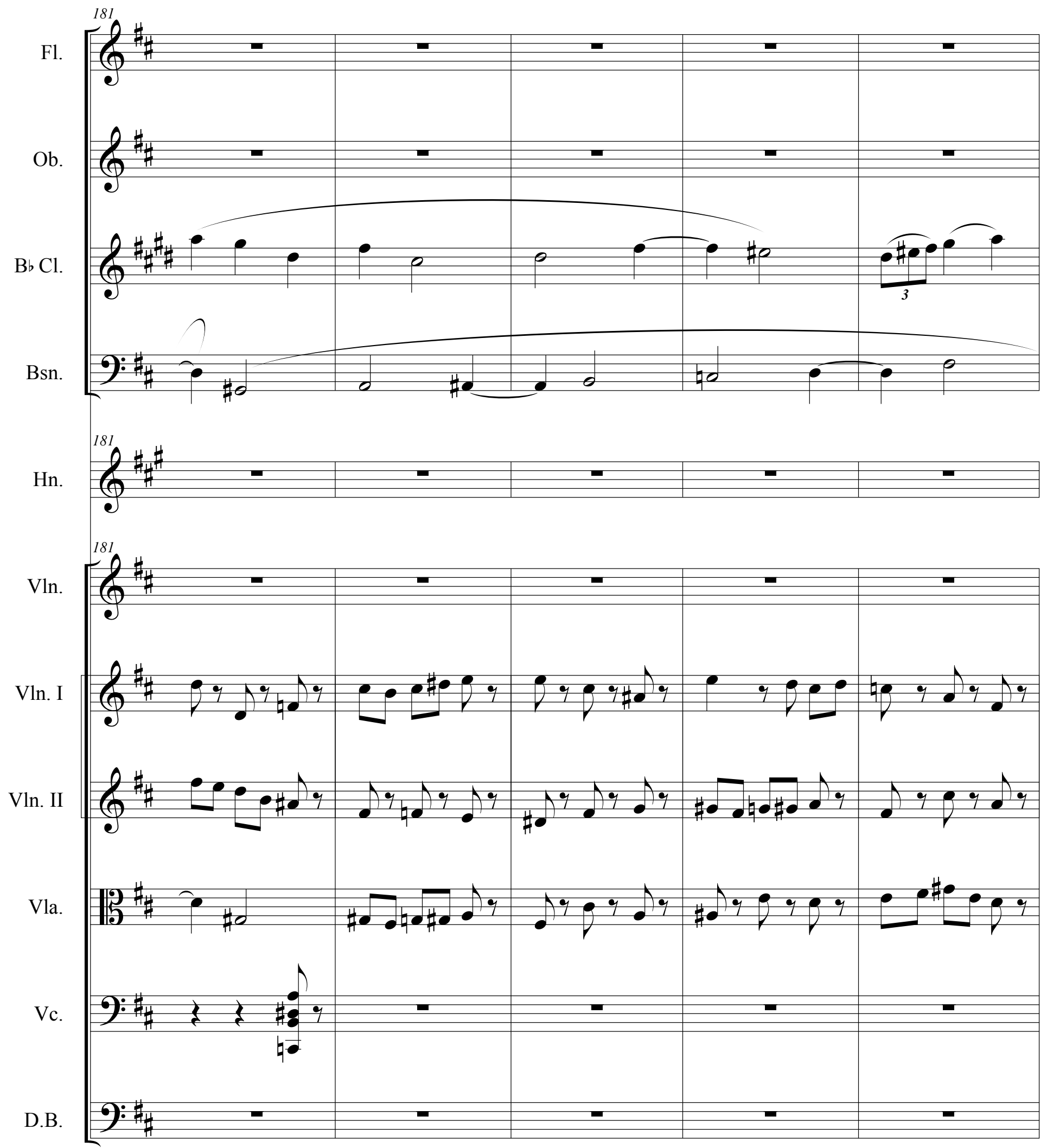
48

For Violin Solo and Chamber Orchestra

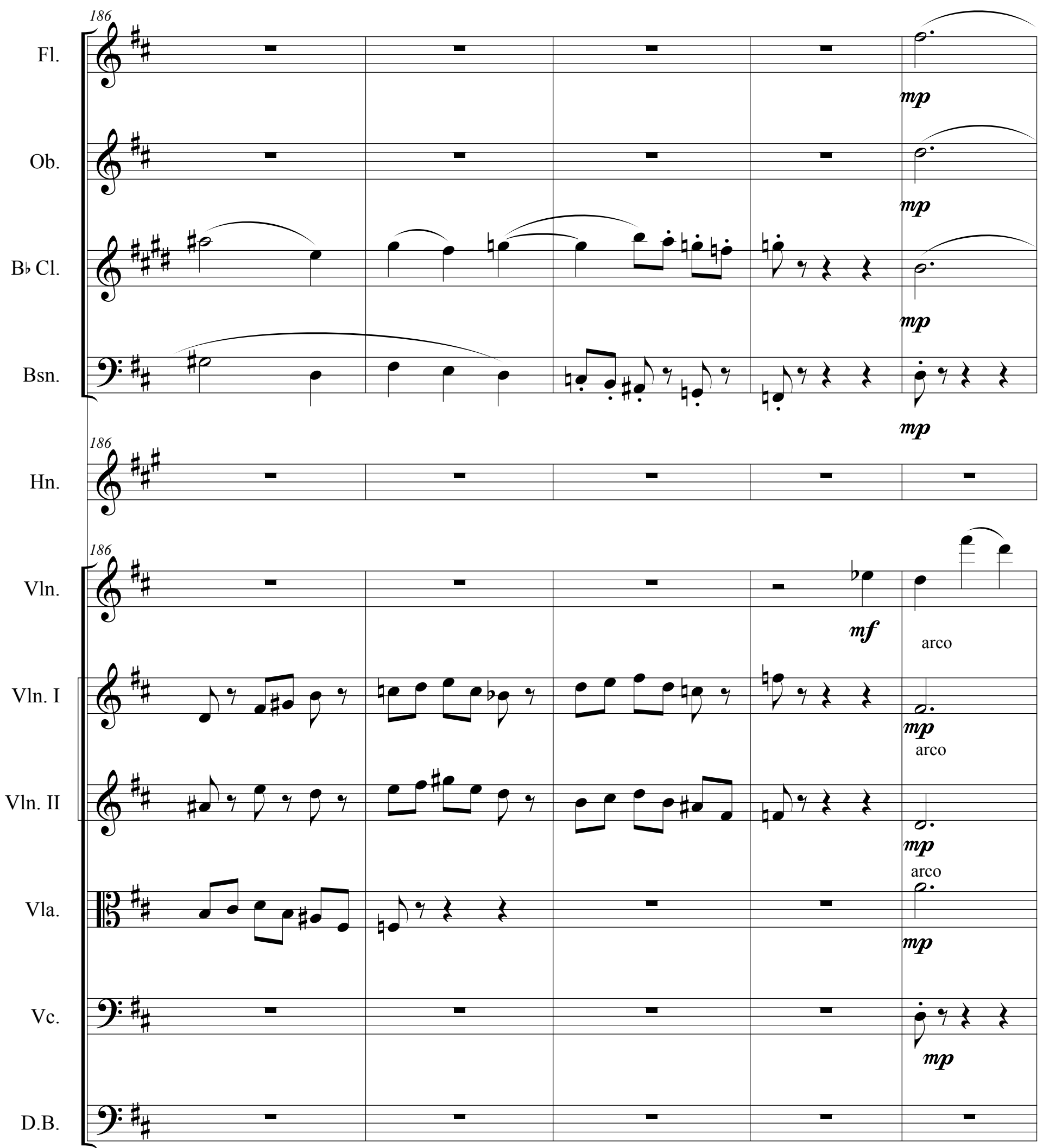




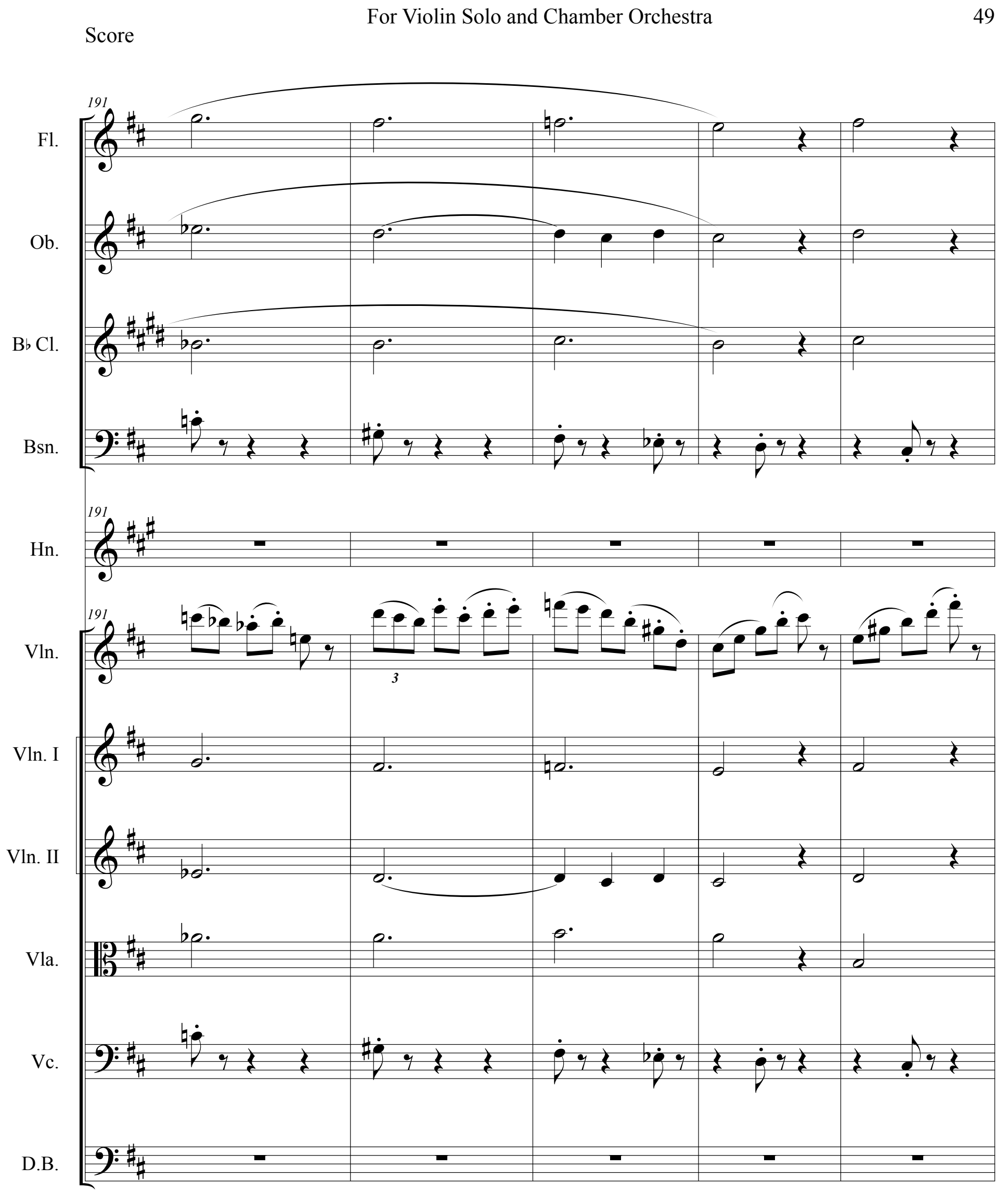


50

For Violin Solo and Chamber Orchestra

Fl.

$\mathrm{Ob}$.

$\mathrm{B} b \mathrm{Cl}$.

Bsn.

Hn.

196

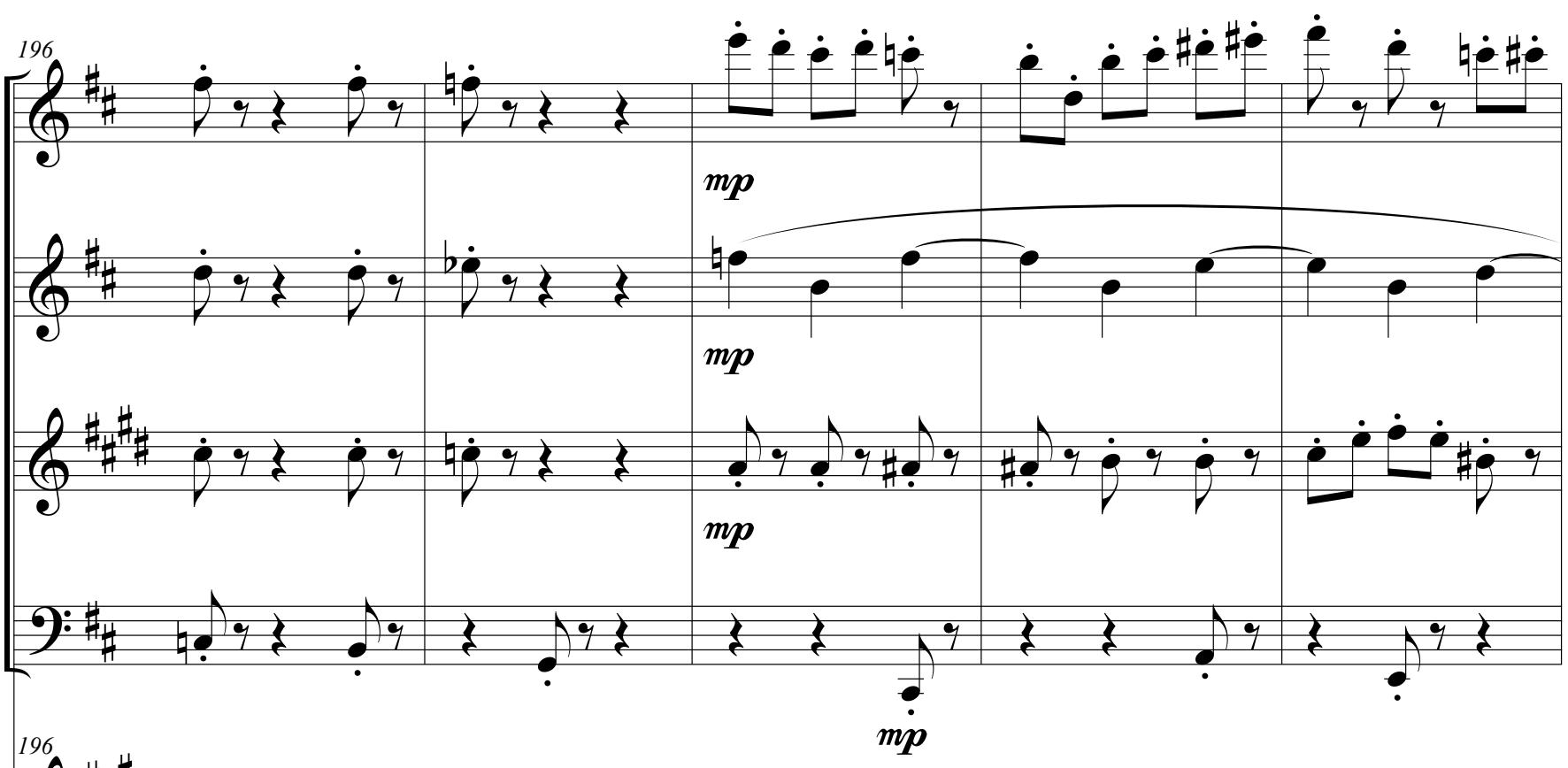

Vln.

Vln. I

Vln. II

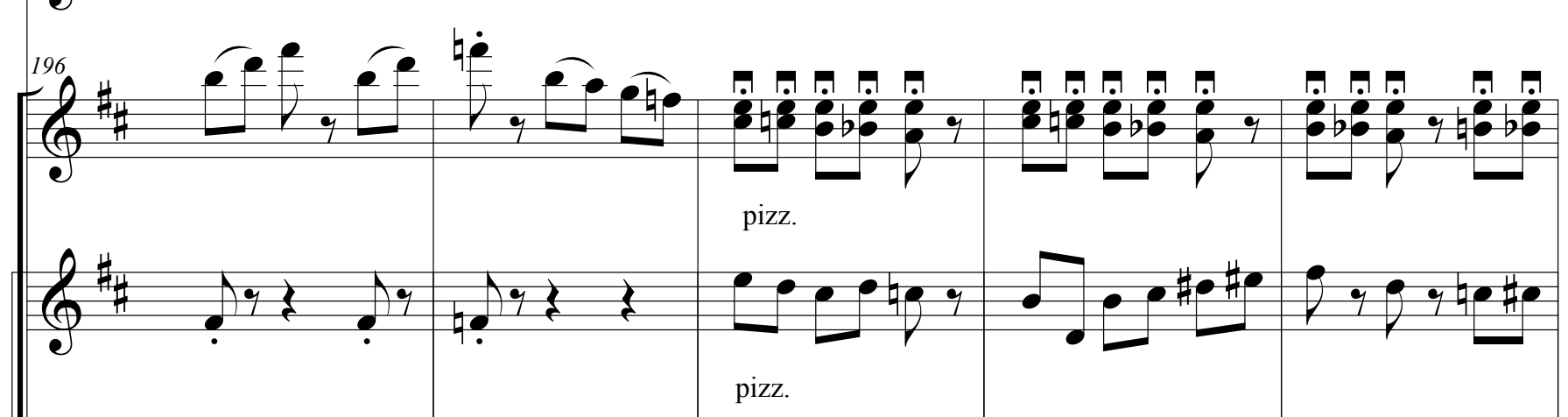

Vla.

Vc.

D.B.

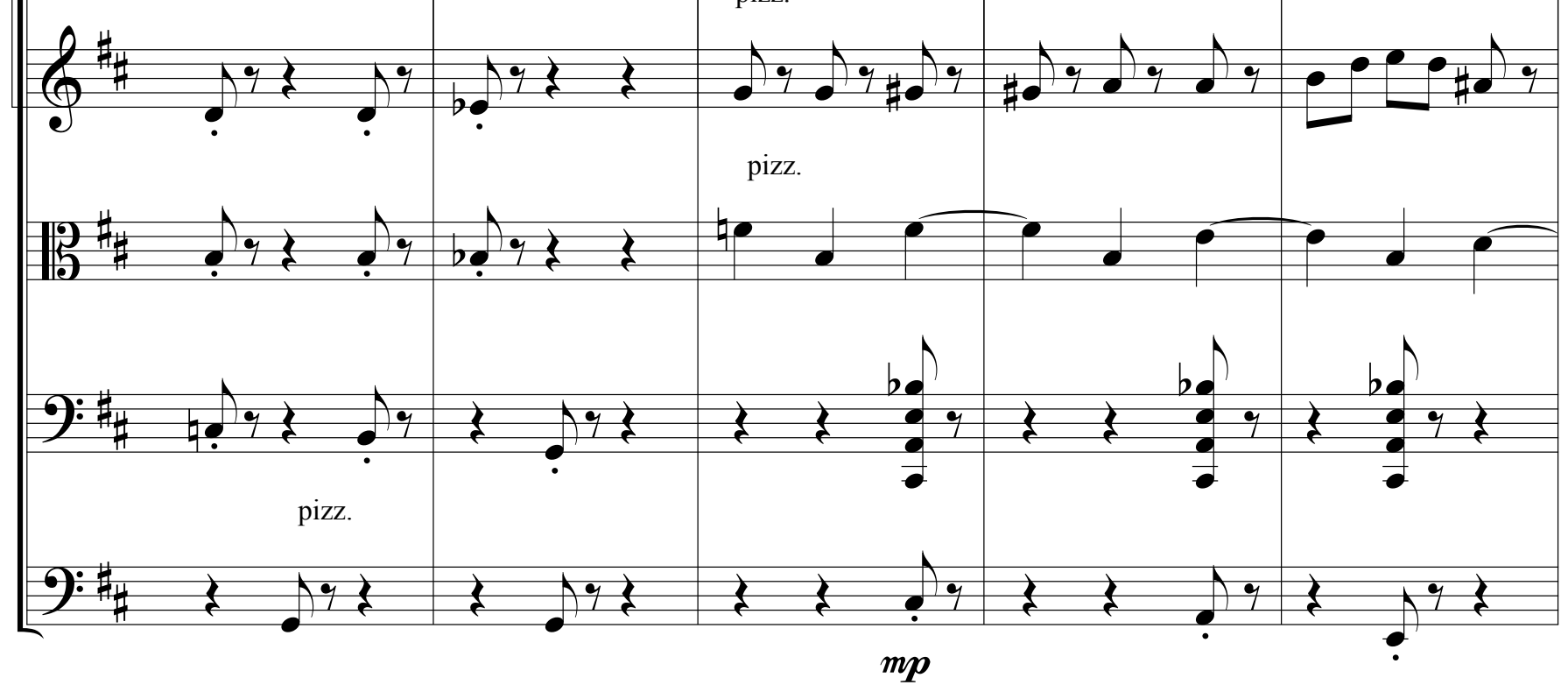


Score

For Violin Solo and Chamber Orchestra

51

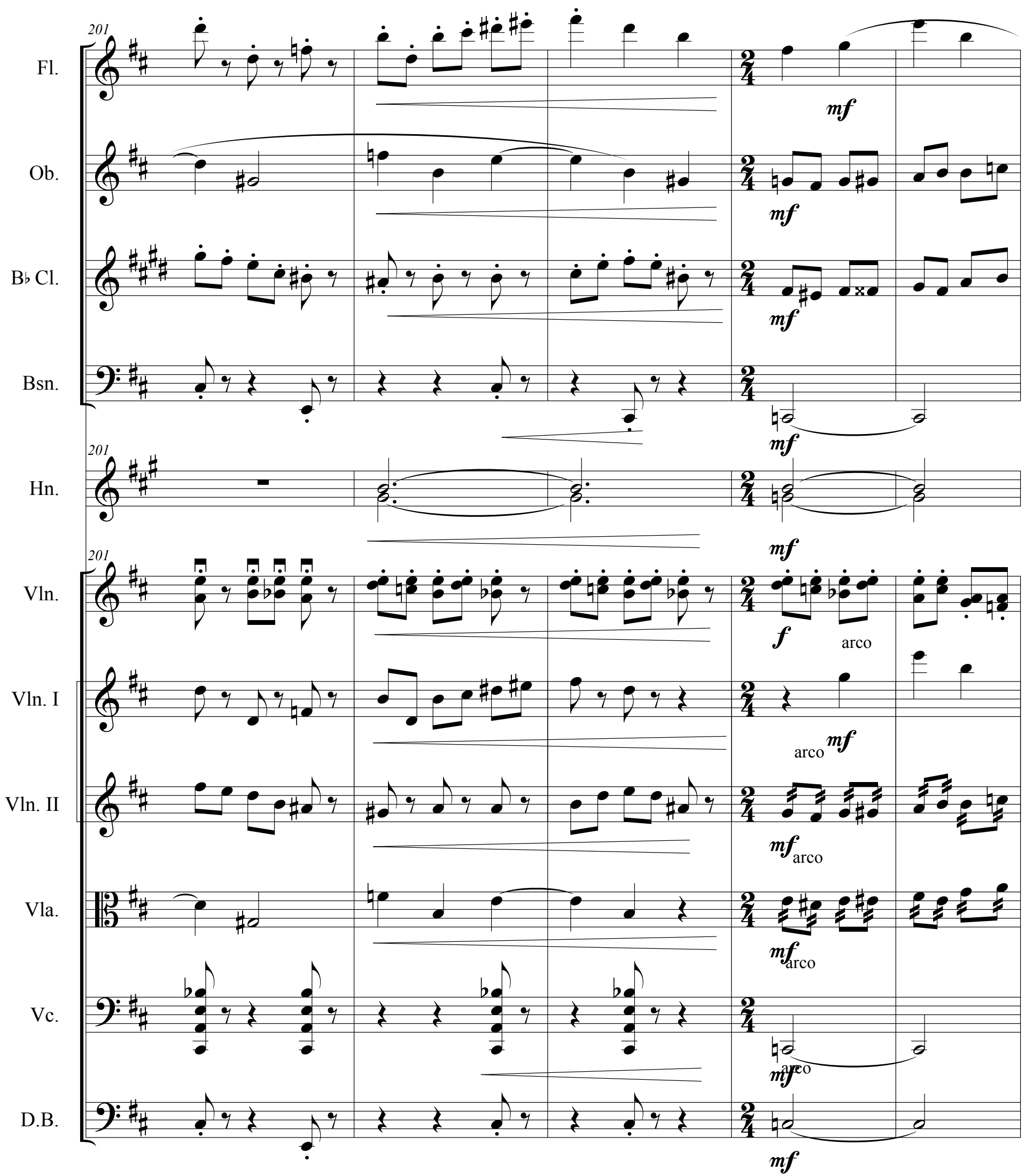




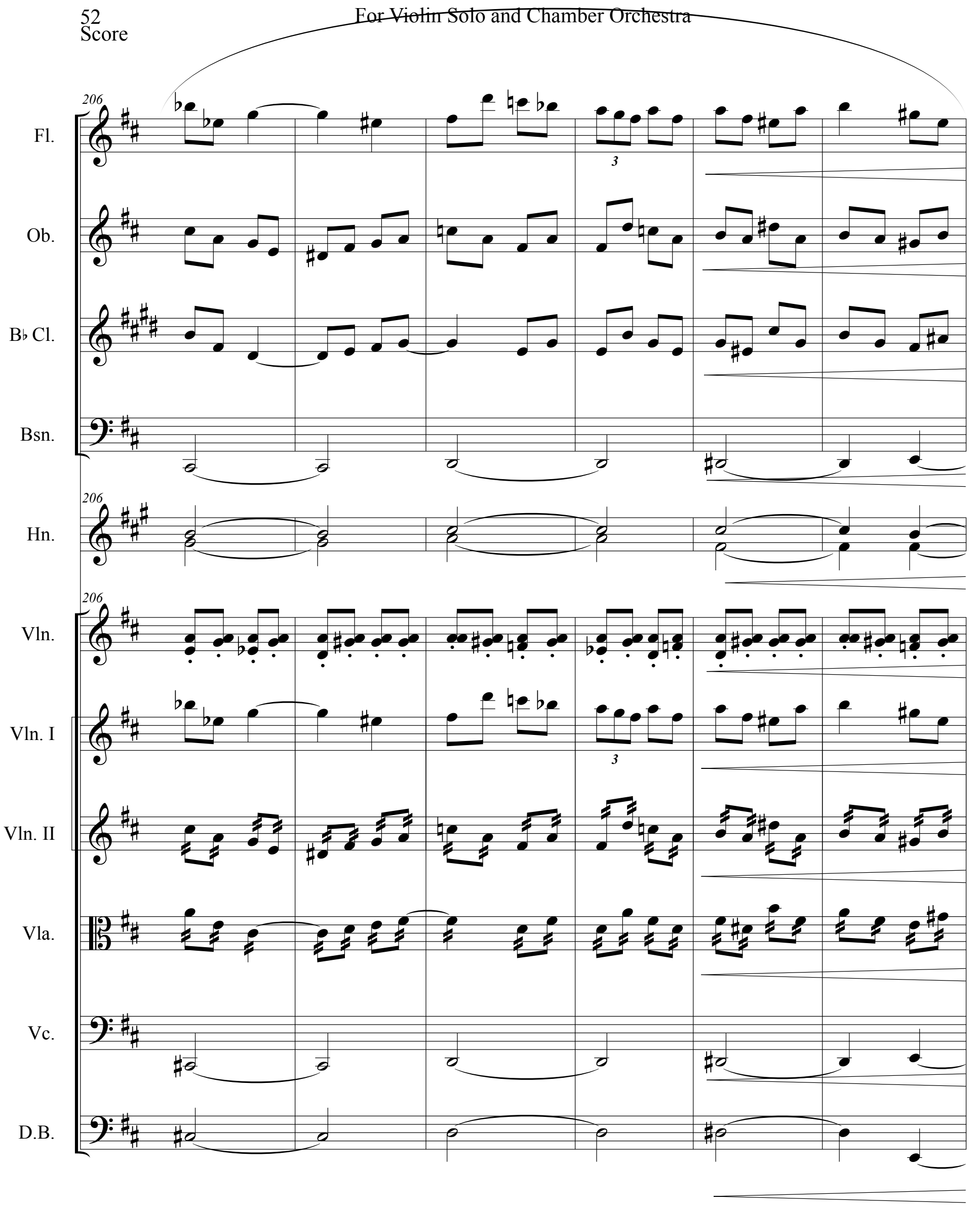


Score

Fl.

212

(9)

$\mathrm{Ob}$

$\theta_{0}^{0}$

$\mathrm{B} b \mathrm{Cl}$.

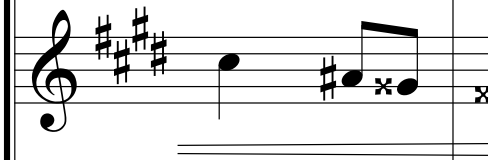

$\$ 0$

Bsn.

0):

\$0

$\int_{0}^{0} 0,0$

$\prod_{0}^{0}$

$\bullet \bullet$

4

Hn.

212

Vln.

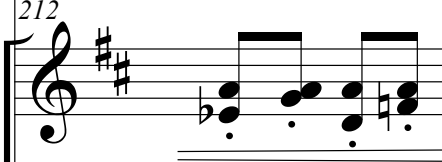

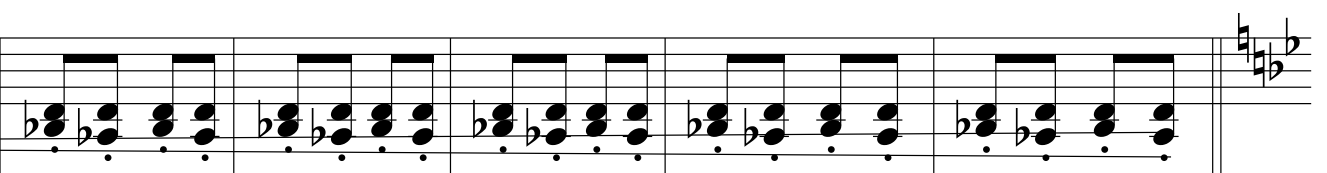

Vln. I

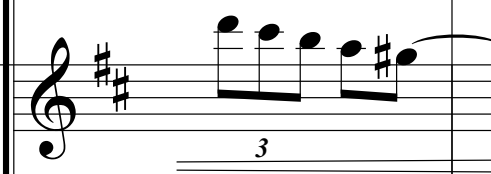

He e e epepe

$+2 \cdot \frac{b}{b}$

Vln. II

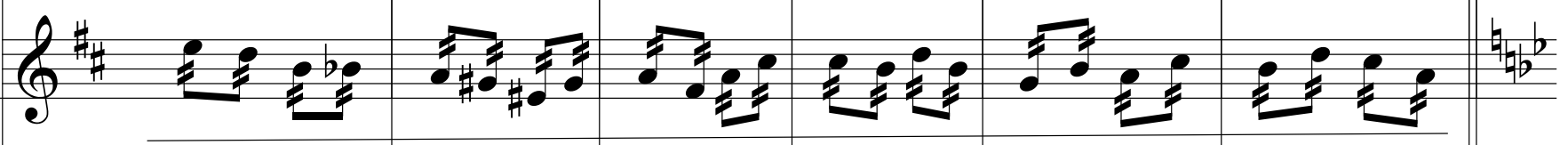

Vla.

H5 :

$2=2$

$2 ; 3$

$\frac{b}{b^{b}}$

Vc

D.B.

9::

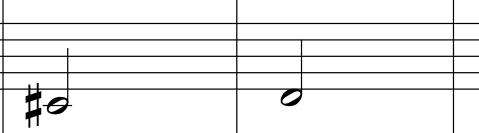

0

$+$

$\frac{b}{b}$

$$
\underline{\underline{0}}
$$

Ho

$+$

-

$+2$ 

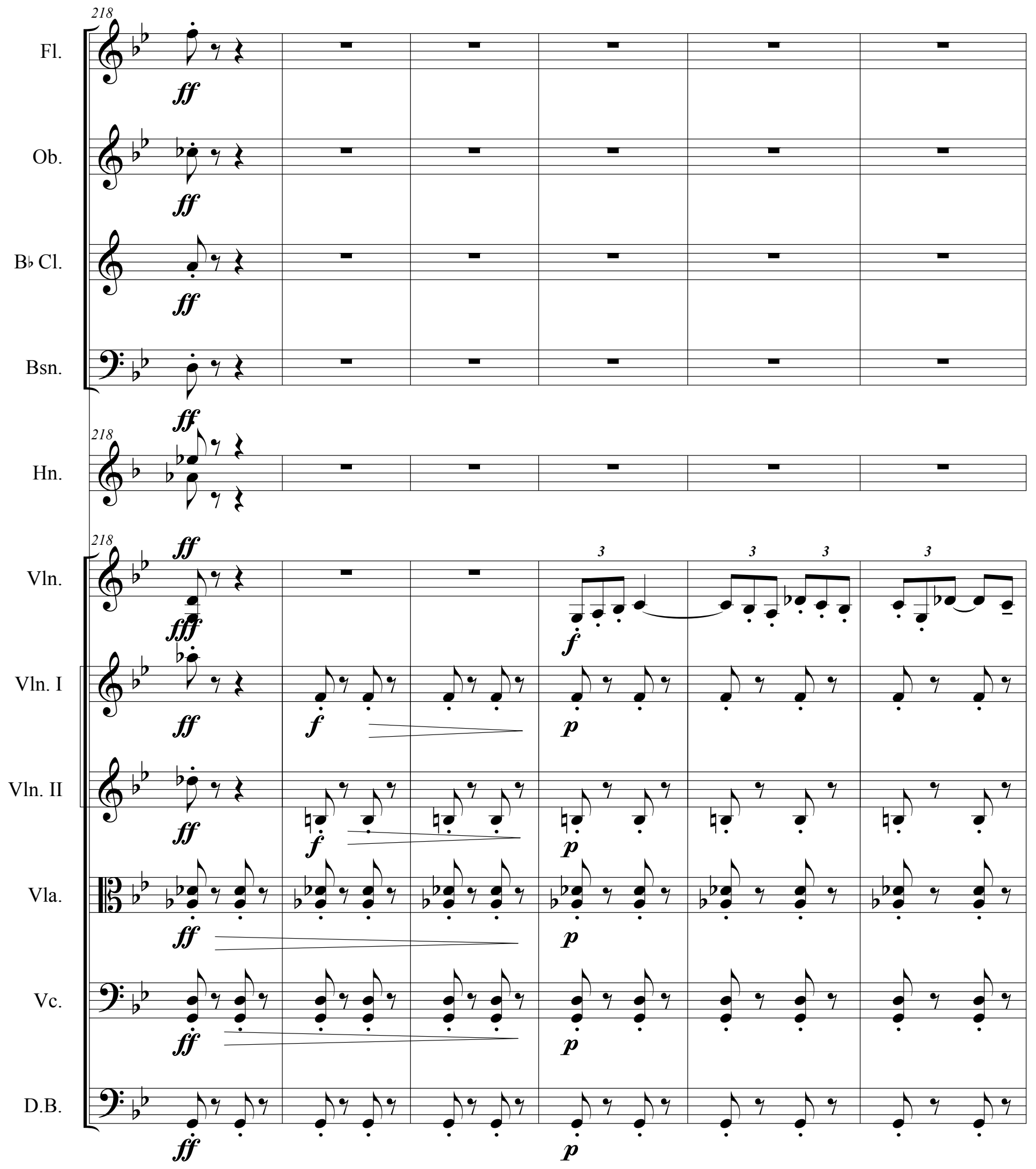


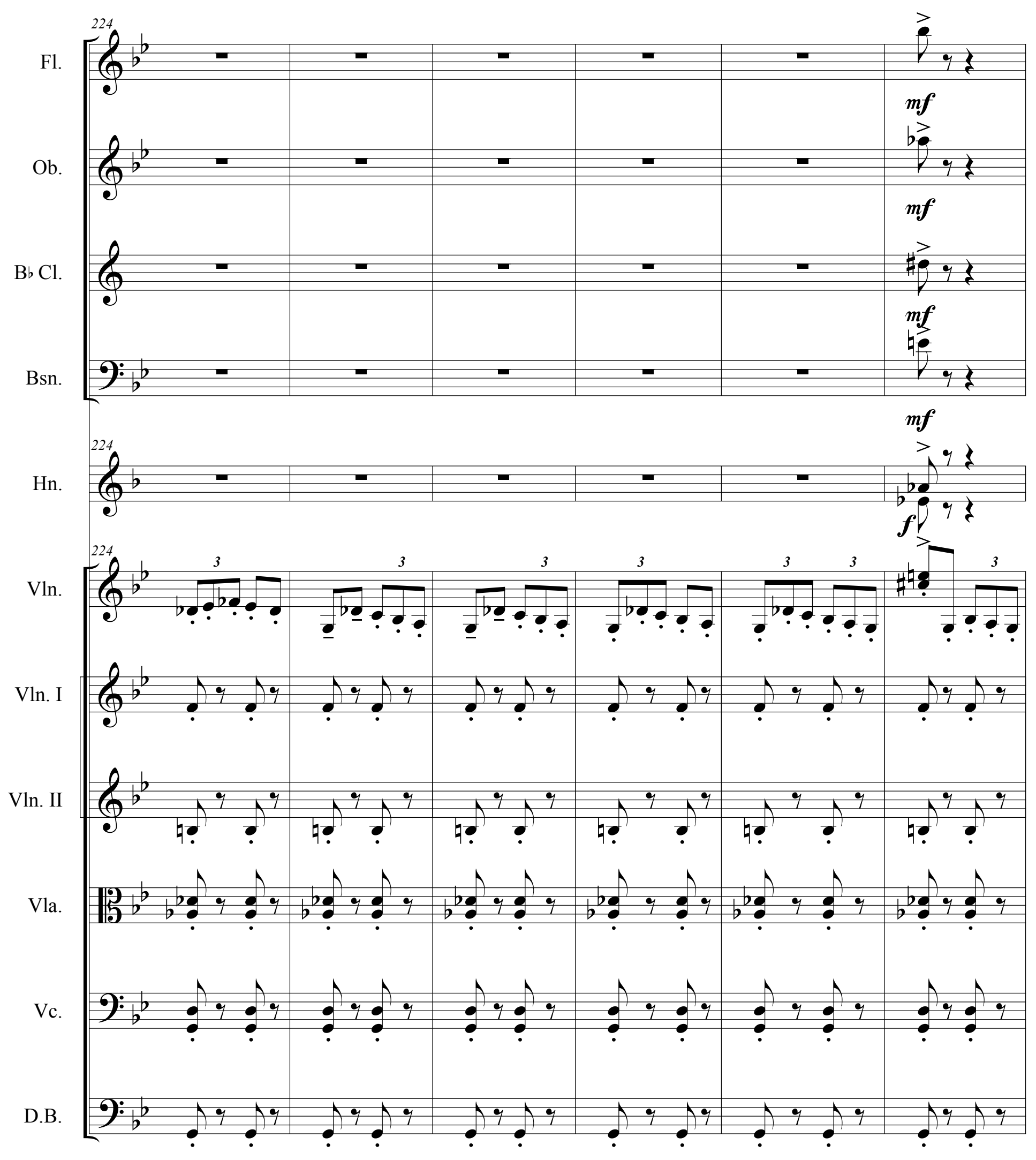


F1.

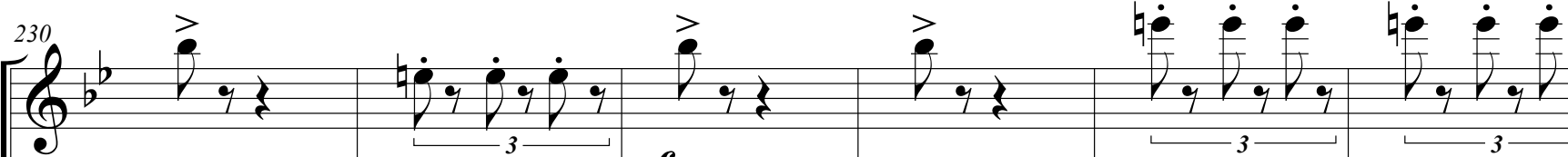

Ob.

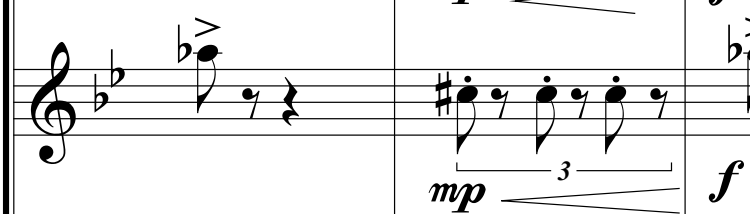
$m \boldsymbol{p}$

$\mathrm{B} b \mathrm{Cl}$.

Bsn.

Hn.

0 $f$

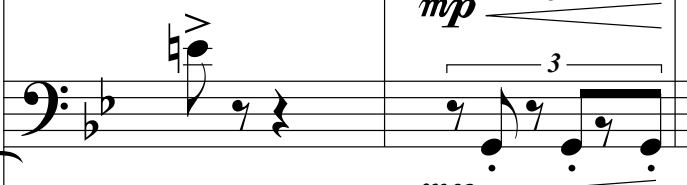
$\boldsymbol{f}_{\text {q }}$

$\operatorname{lig}^{>}$ $f$

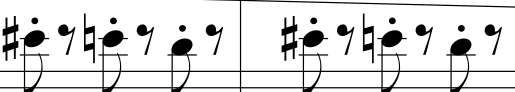
be $\left(\begin{array}{c}g^{230} \\ b^{b}\end{array}\right.$

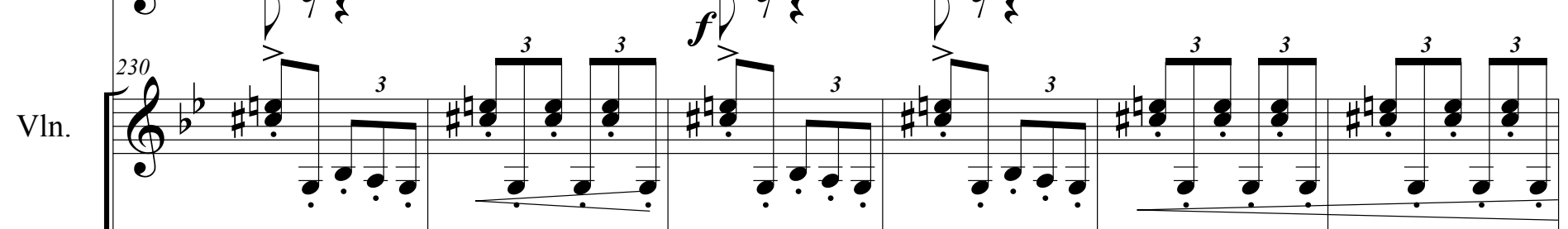

Vln. I

$\left(a b^{b}\right) a$

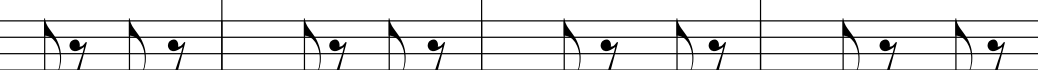
? ? ? ?

Vln. II

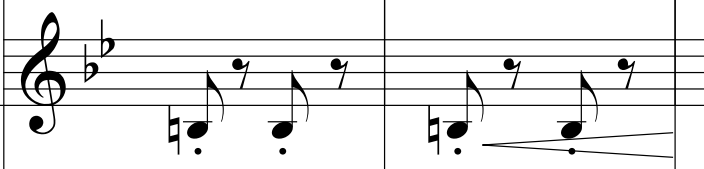

Vla.

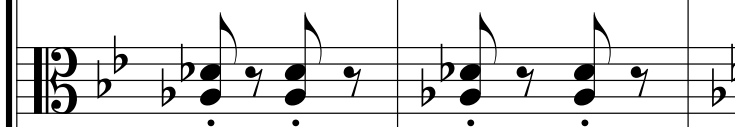
mf $\dot{p}$

Vc.

D.B.

$0: \frac{b}{b}: 9 ?$

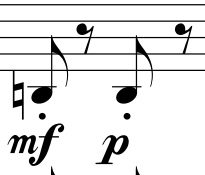
$\left.\prod_{9}^{9}\right)^{9}$ $\left.\int^{4} \int^{4}\right)^{4} \overbrace{}^{4}$

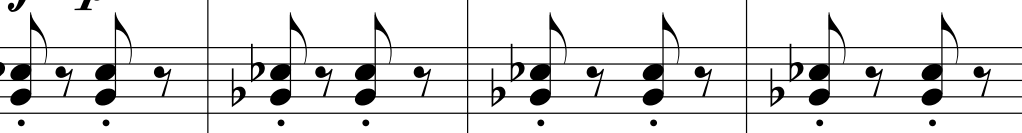
mf $\boldsymbol{p}$

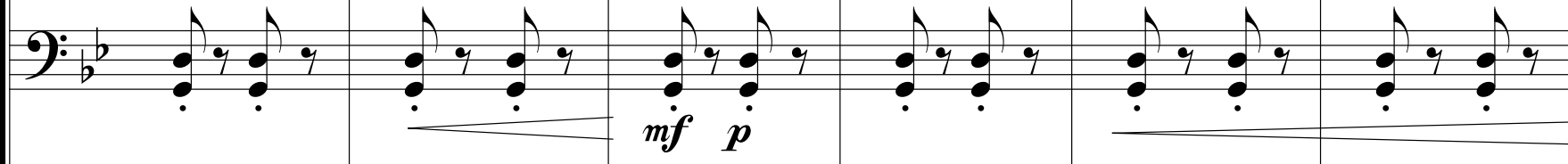
$\ddot{m f}: \dot{p}$ 


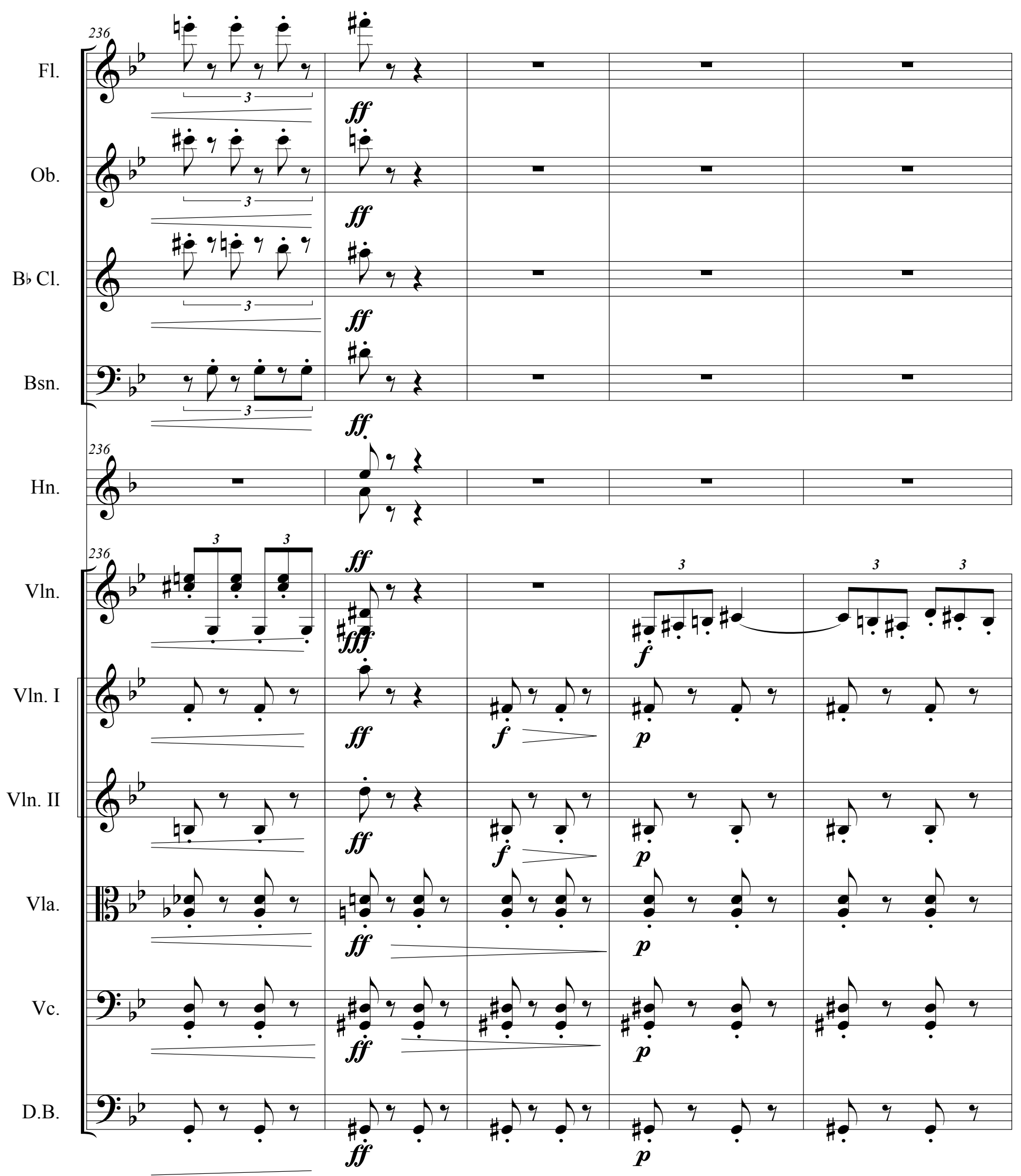



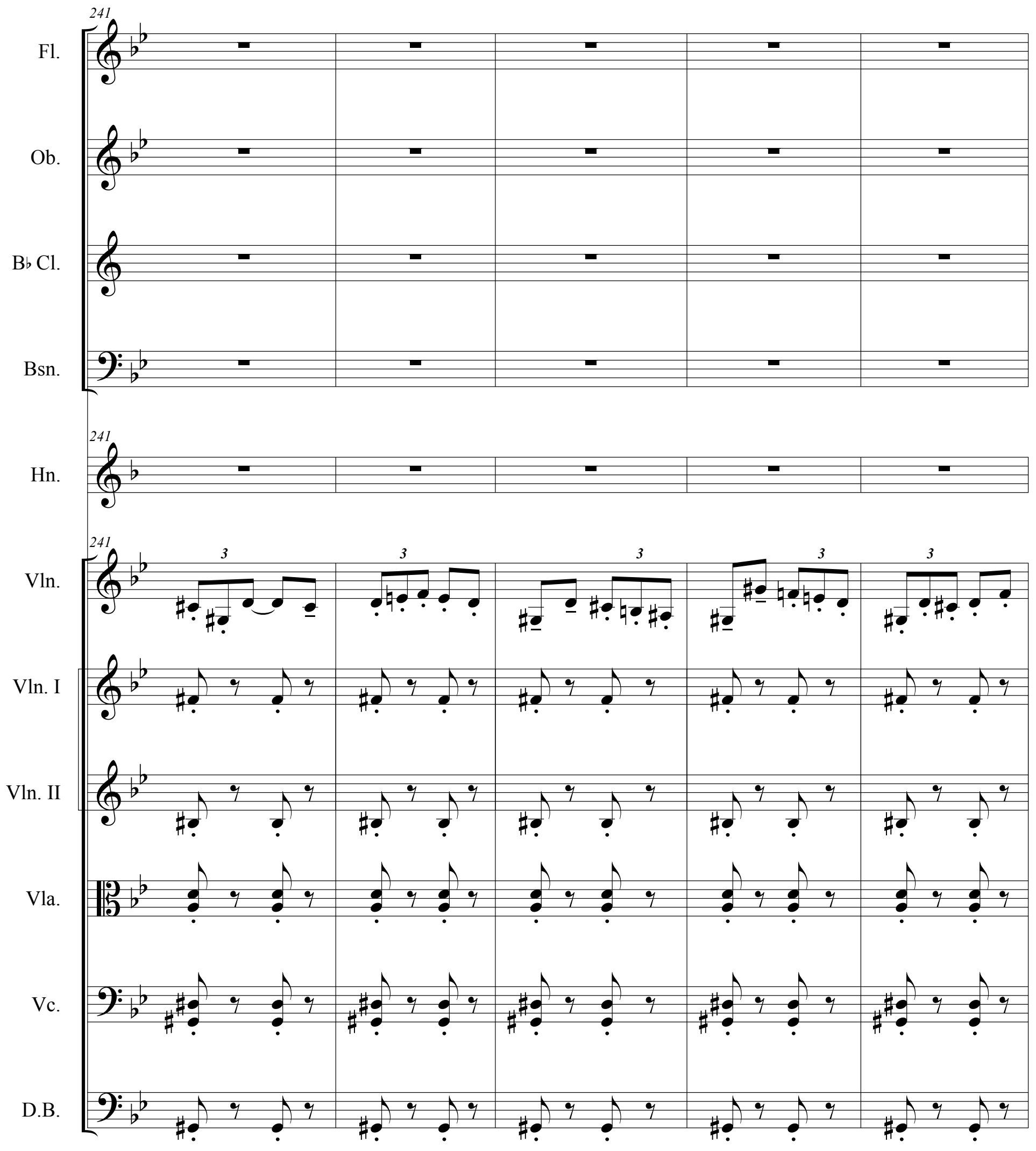


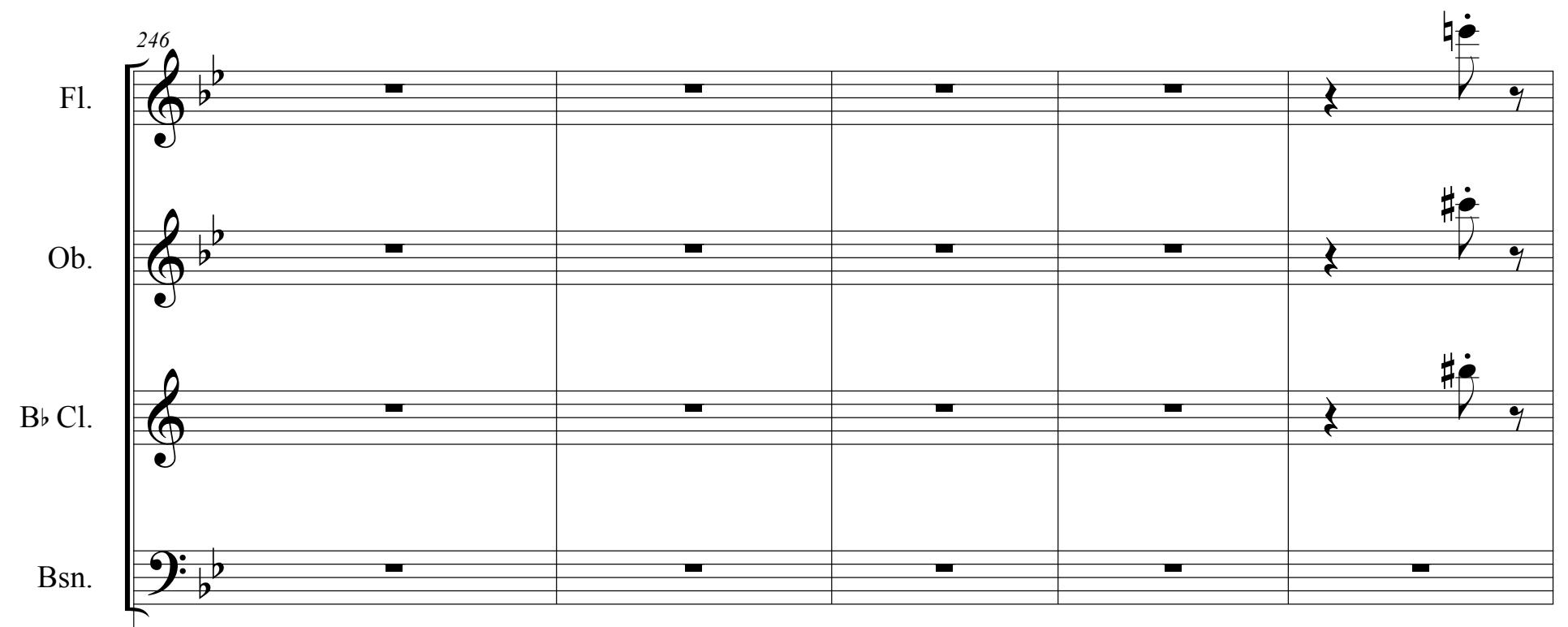

Hn.
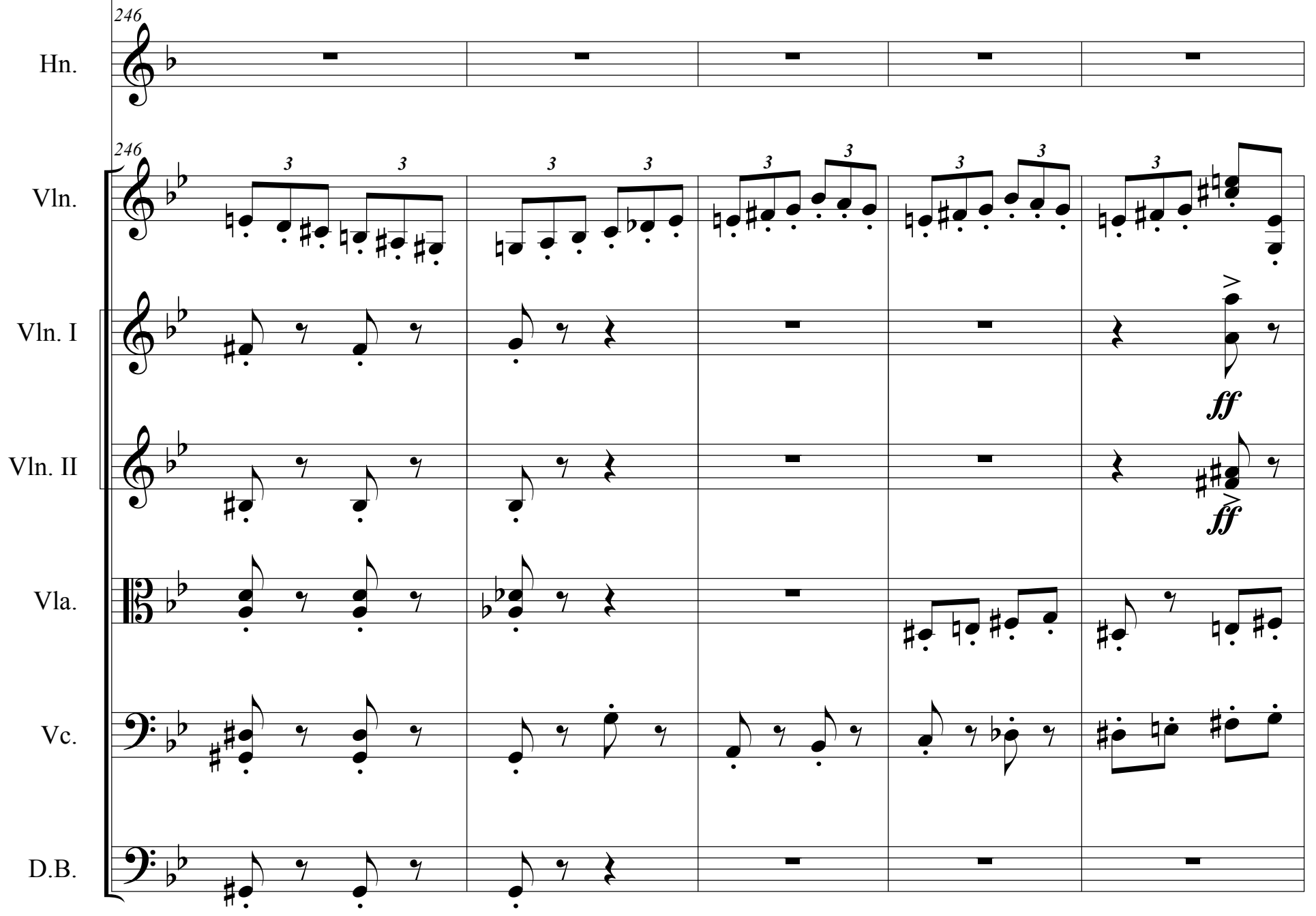
60

For Violin Solo and Chamber Orchestra

F1.

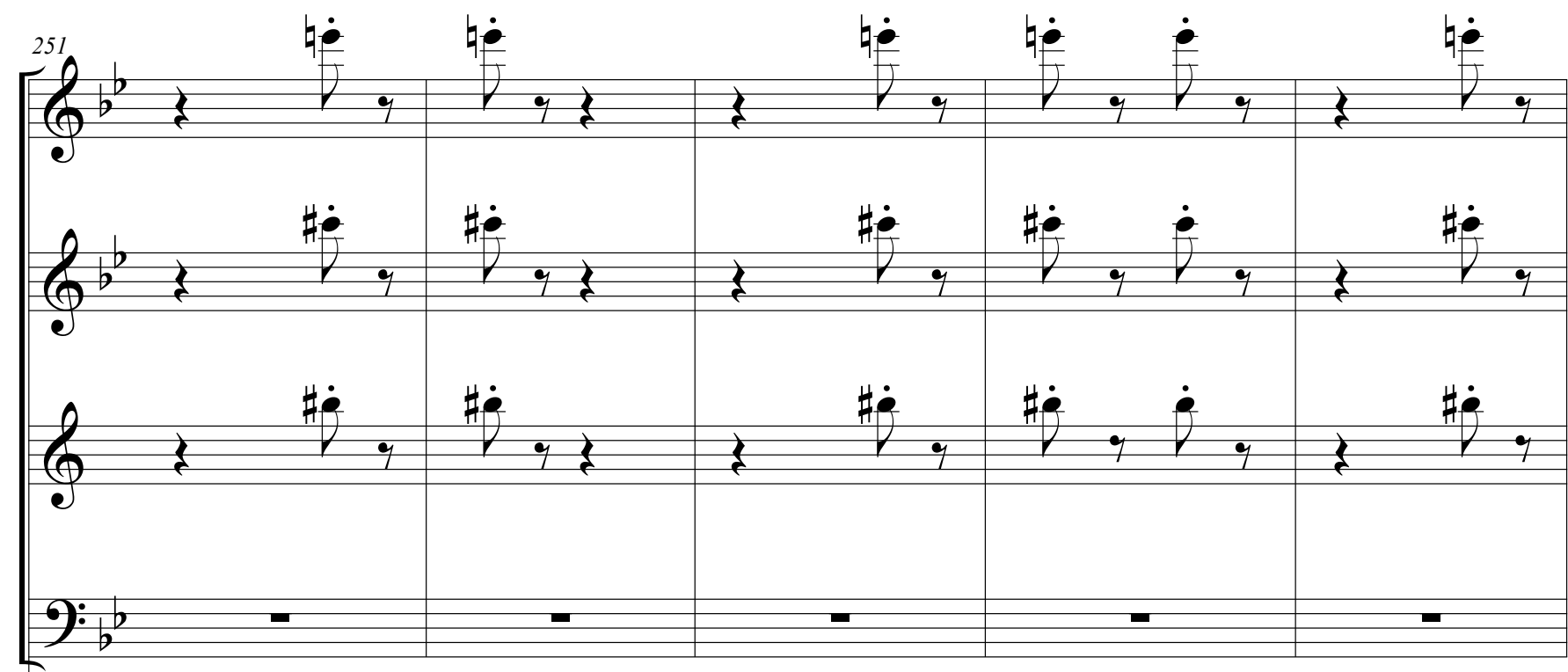

$\mathrm{B} b \mathrm{Cl}$.

Ob.

Bsn.

Hn.

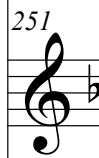

Vln.

Vln. I

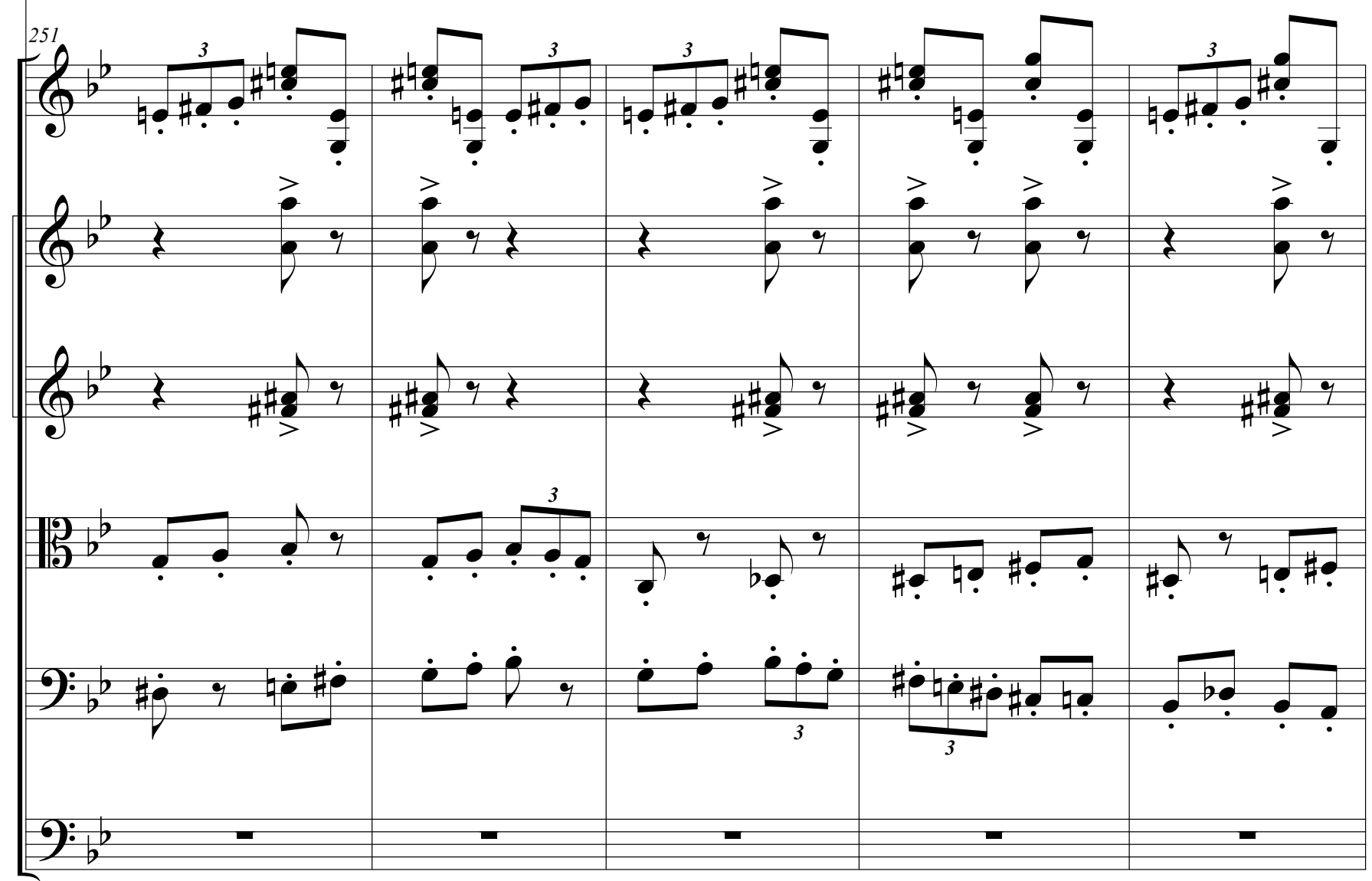

Vla.

Vc.

D.B. 

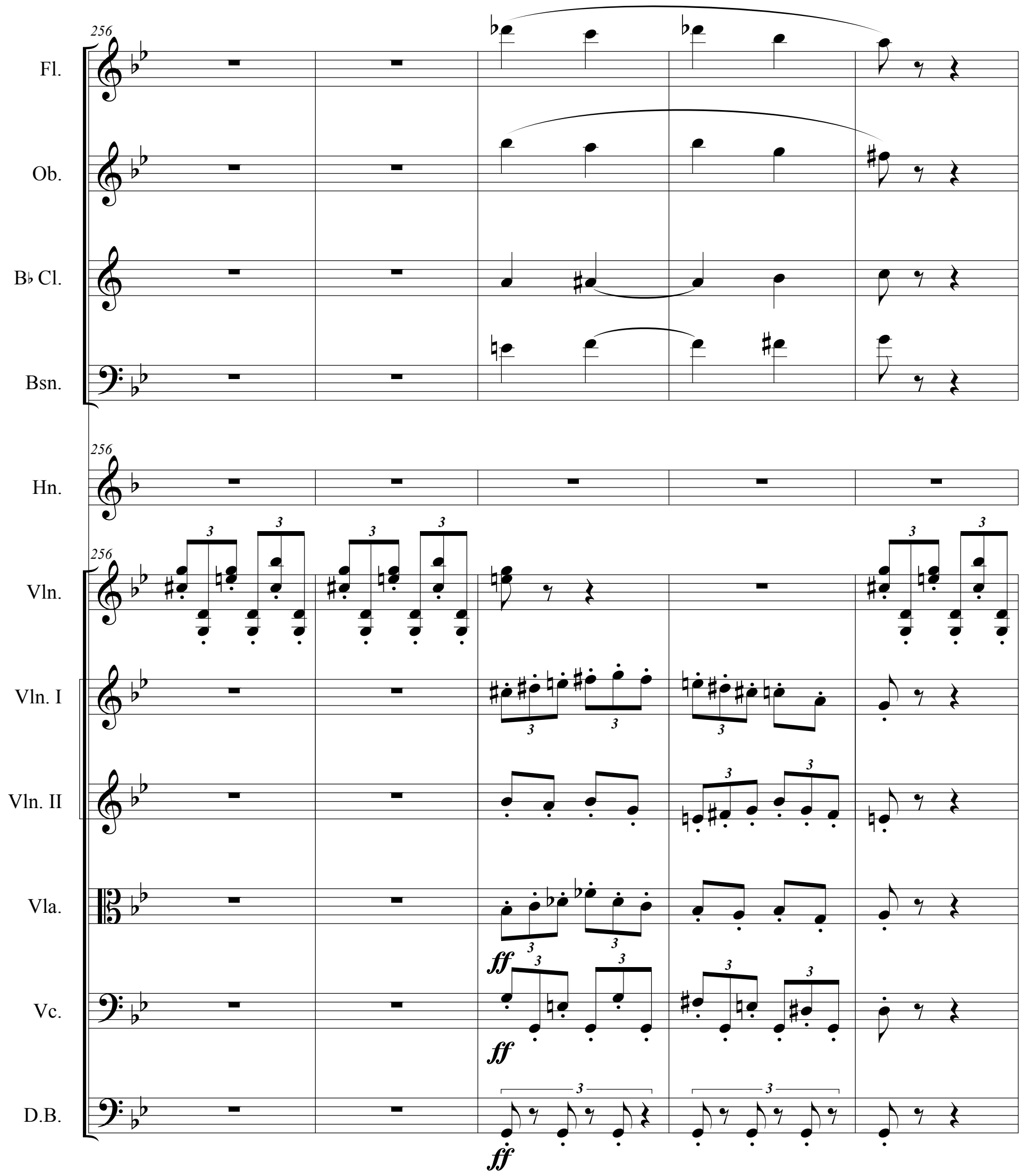
Fl.

$\mathrm{B}$ b $\mathrm{Cl}$.

$\mathrm{Ob}$.

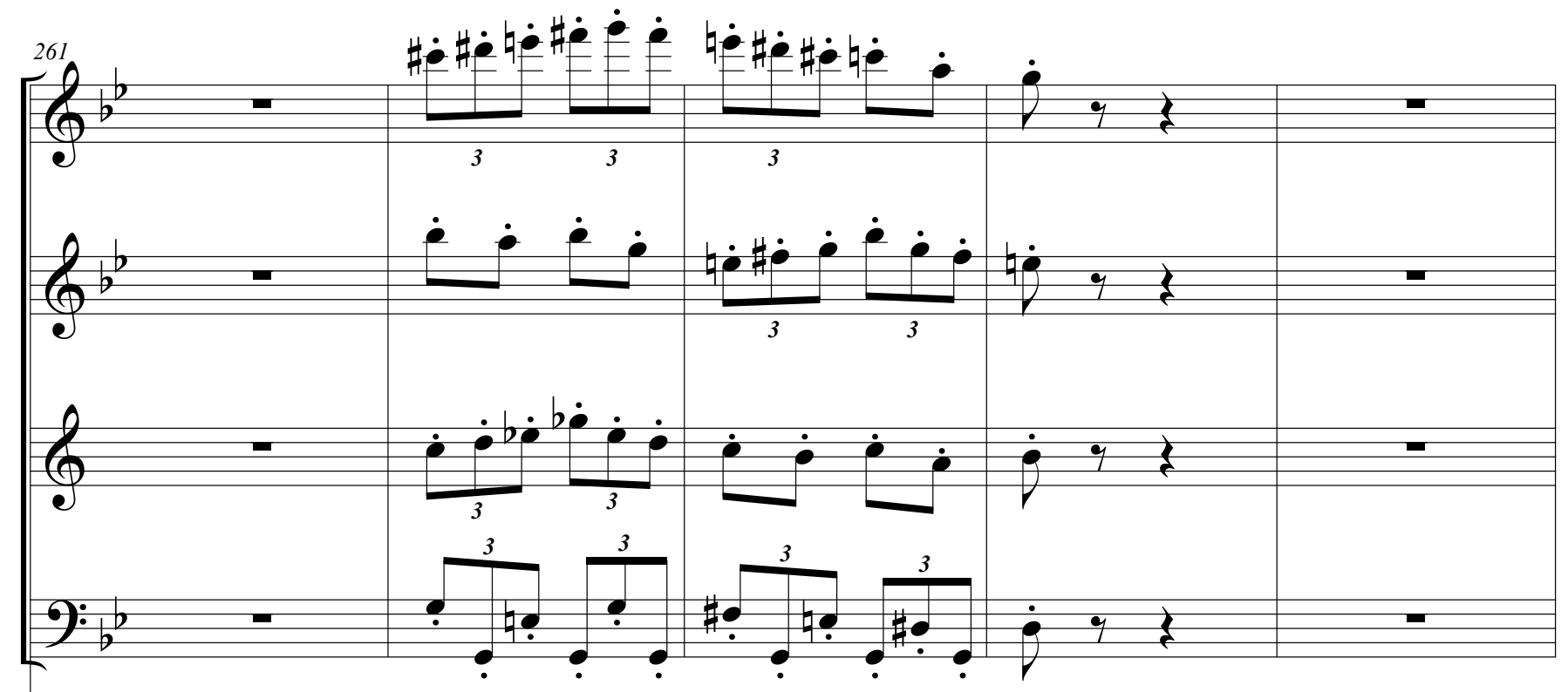

Bsn.

Hn.
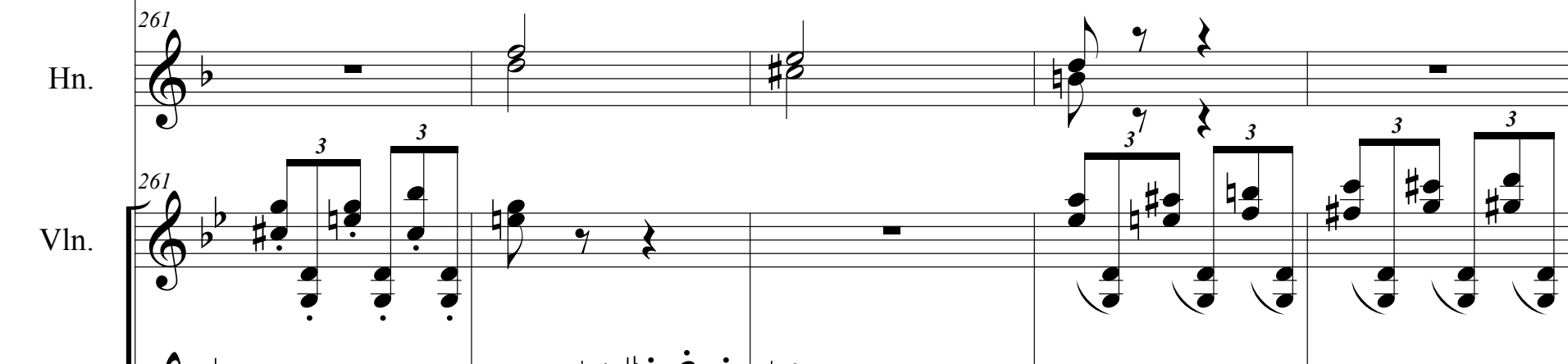

Vln. I

Vln. II

$a^{b^{b}}=$

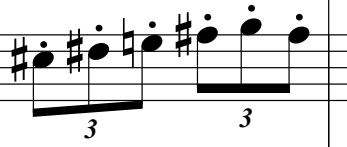

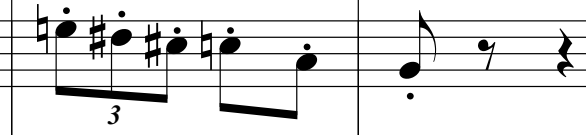

Vla.

$0^{b^{b}}=$

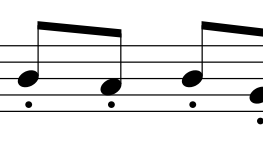

$\div$

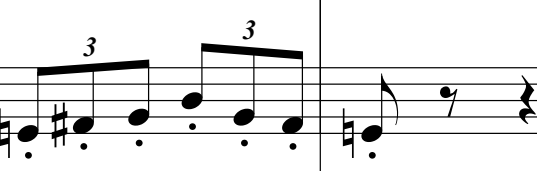

Vc.

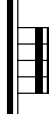

皮

$b_{b}^{b} b \dot{b} b \dot{0} \dot{0} \square-\square$

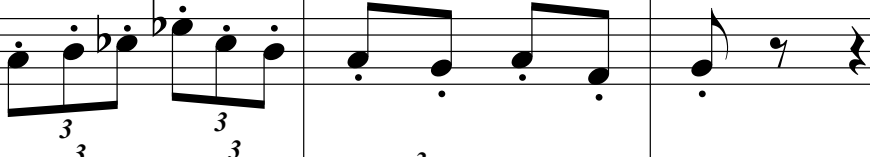

D.B.

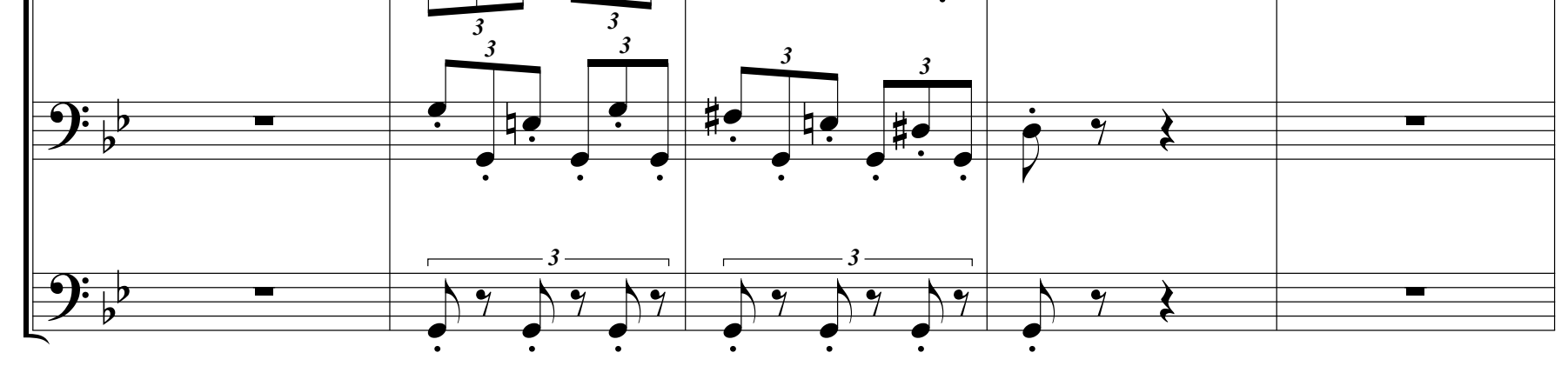




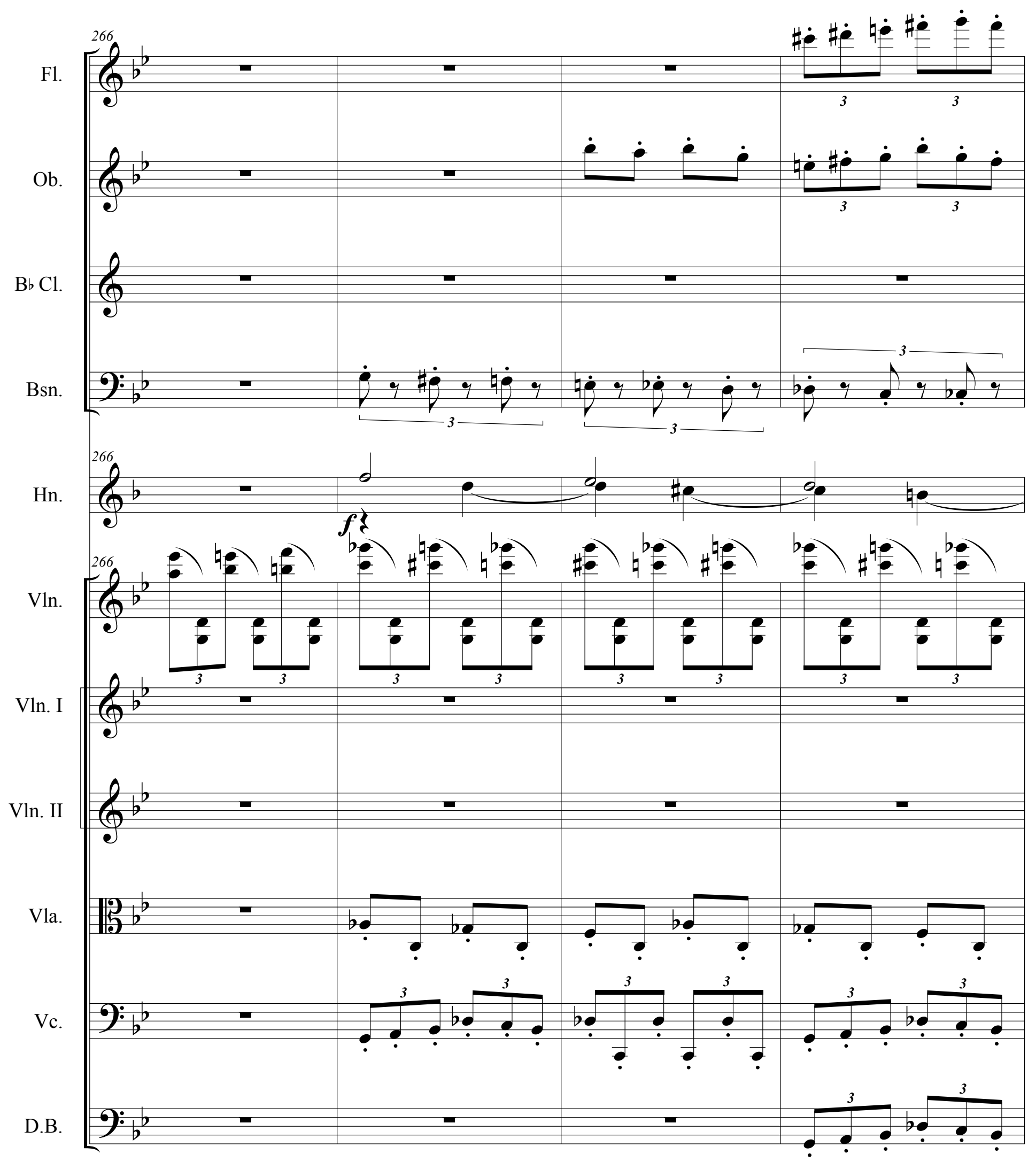


Fl.

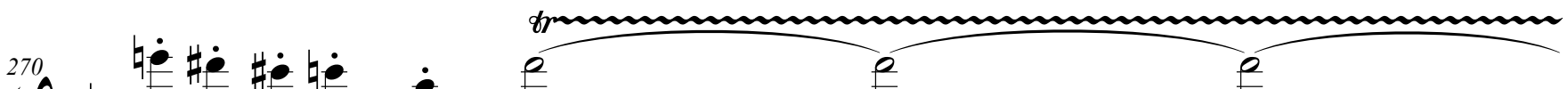

$\mathrm{Ob}$.

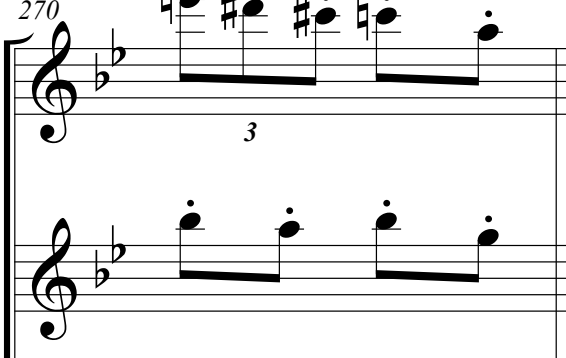

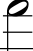
e 2 he

$\mathrm{B} b \mathrm{Cl}$.

Bsn.

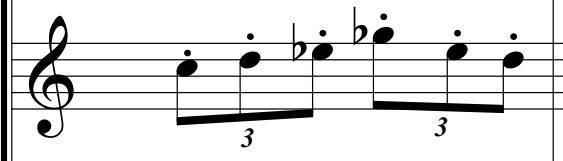

cresc.

grom 0

cresc.

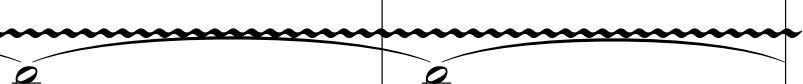

$\frac{b}{b} \int_{l}^{b}$

Hn.

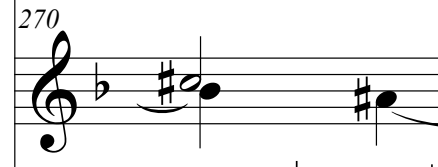

10

He

100

19

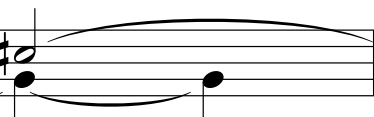

Vln.

Vln. I 270

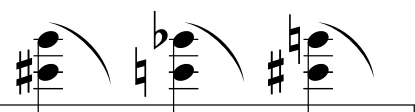

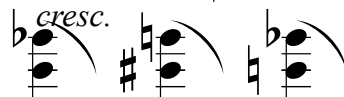

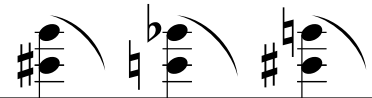
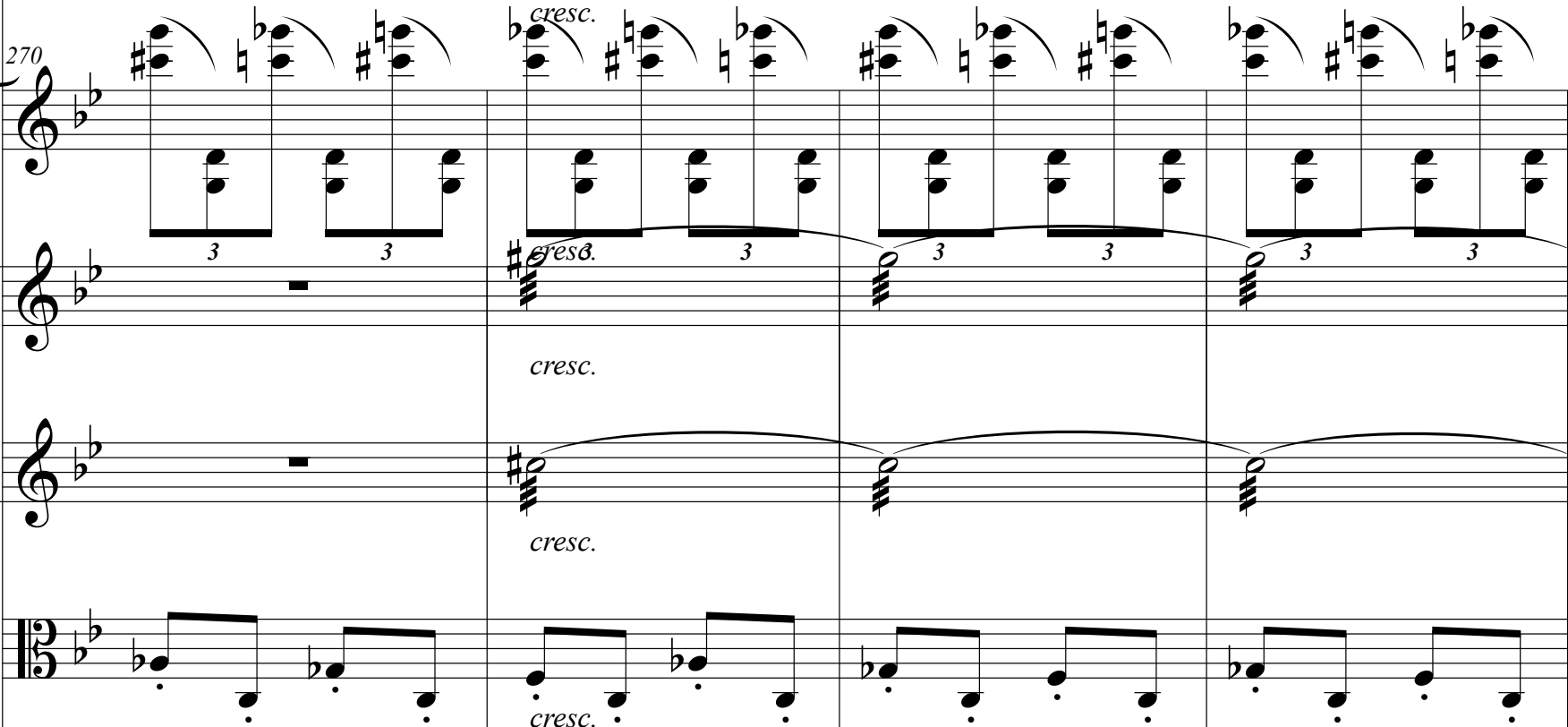

Vla.

Vln. II

Vc.

D.B.

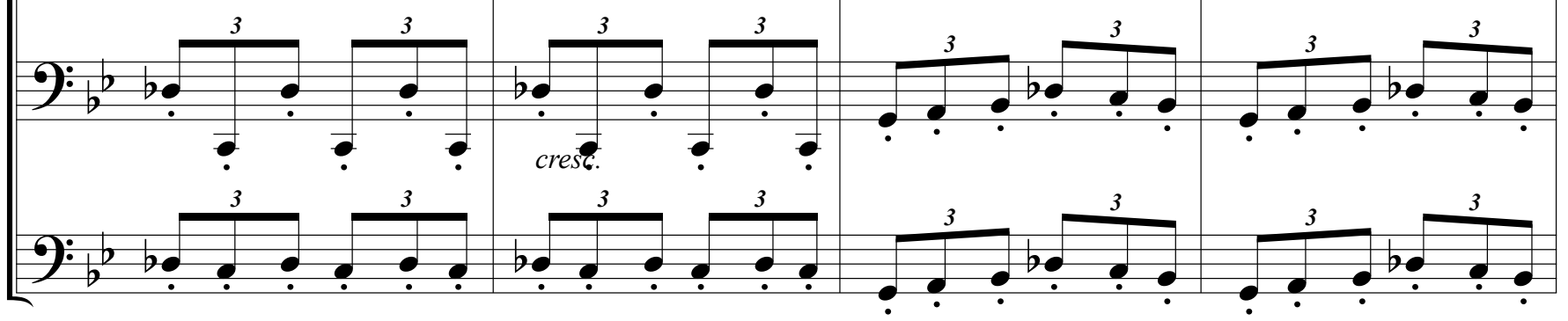

cresc. 
Score

Fl.

$\mathrm{Ob}$.

$\mathrm{B} b \mathrm{Cl}$.

Hr.

Ven.

Ven. I

VIi. II

Via.

Vc.

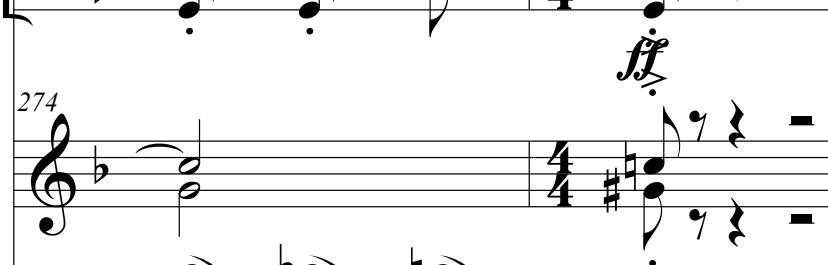

Quarter $=120$

$\dot{\mathscr{f}}$

7
7
8
8
8

$\boldsymbol{f}$

$b=0$
For Violin Solo and Chamber Orchestra

65

Poco Keno Miso
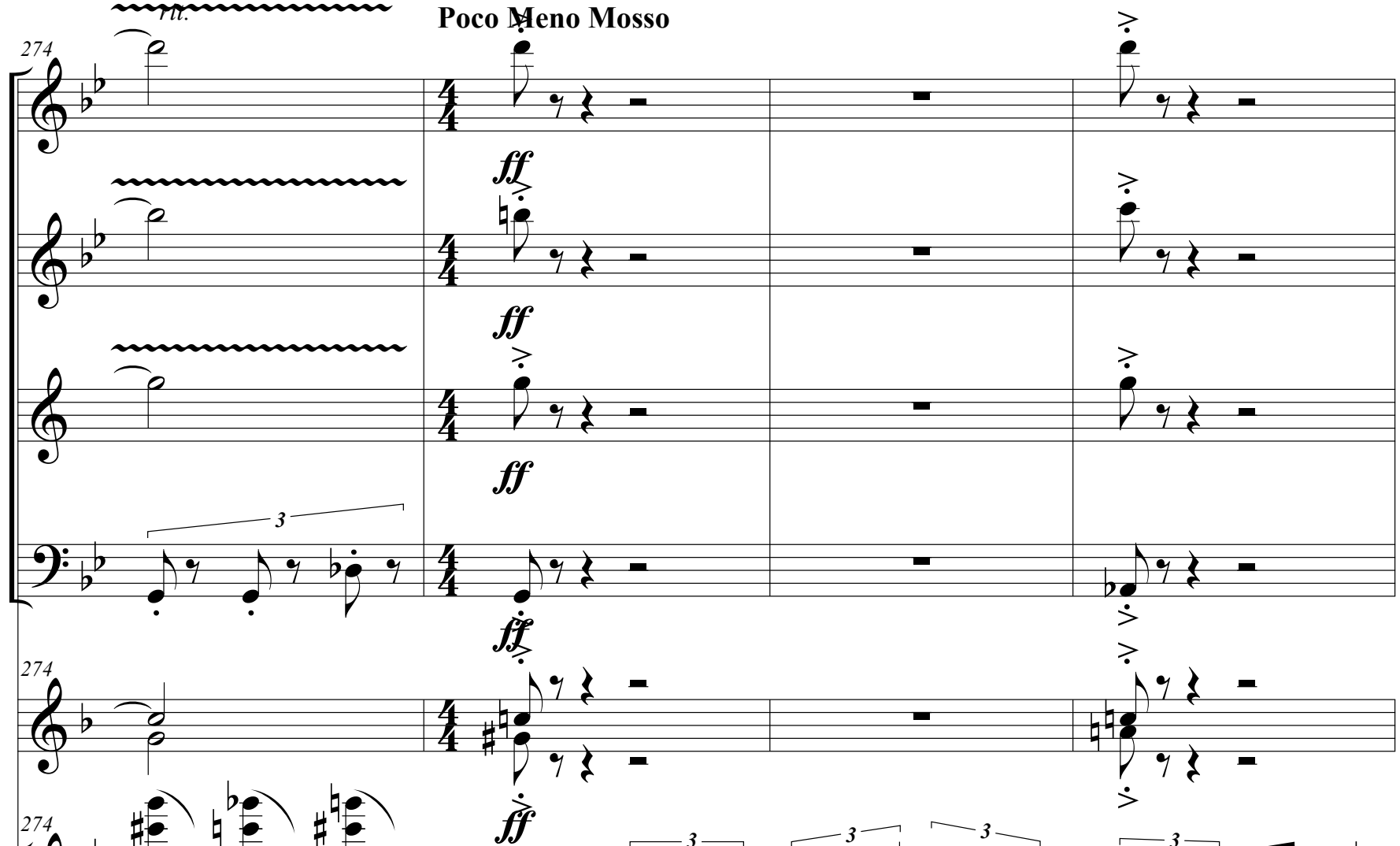

$41=$

$-3 \longrightarrow$

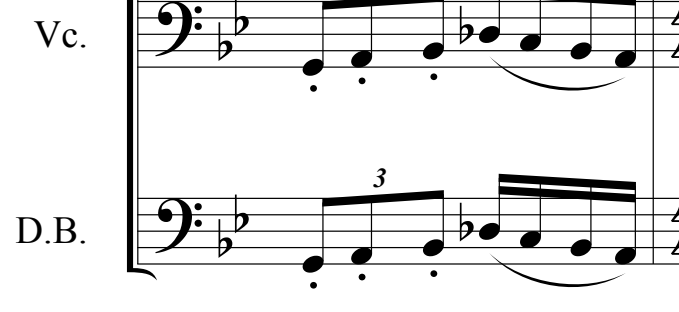

pizz.

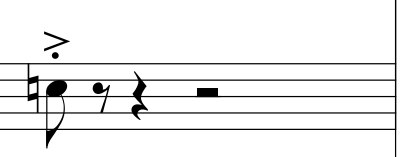

$\vec{f}$

$\stackrel{4}{9}$

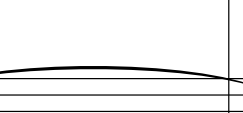

8

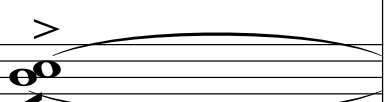

$m p$

$?$

$\dot{f}$ 
66

For Violin Solo and Chamber Orchestra

Fl.

Ob.

$\mathrm{B} b \mathrm{Cl}$.

Hon.

Bn.

Ven.

YIn. I
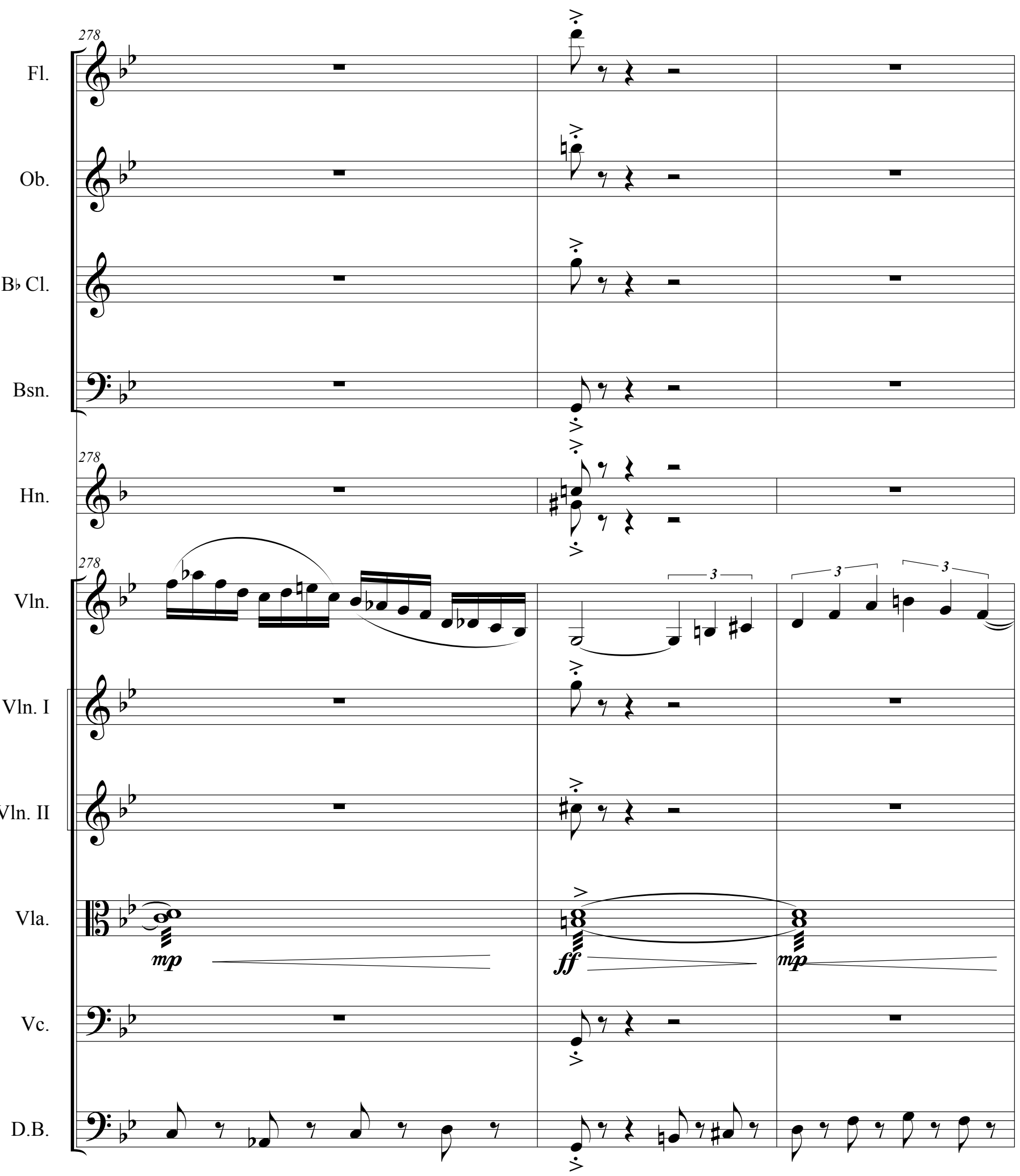
Score

For Violin Solo and Chamber Orchestra

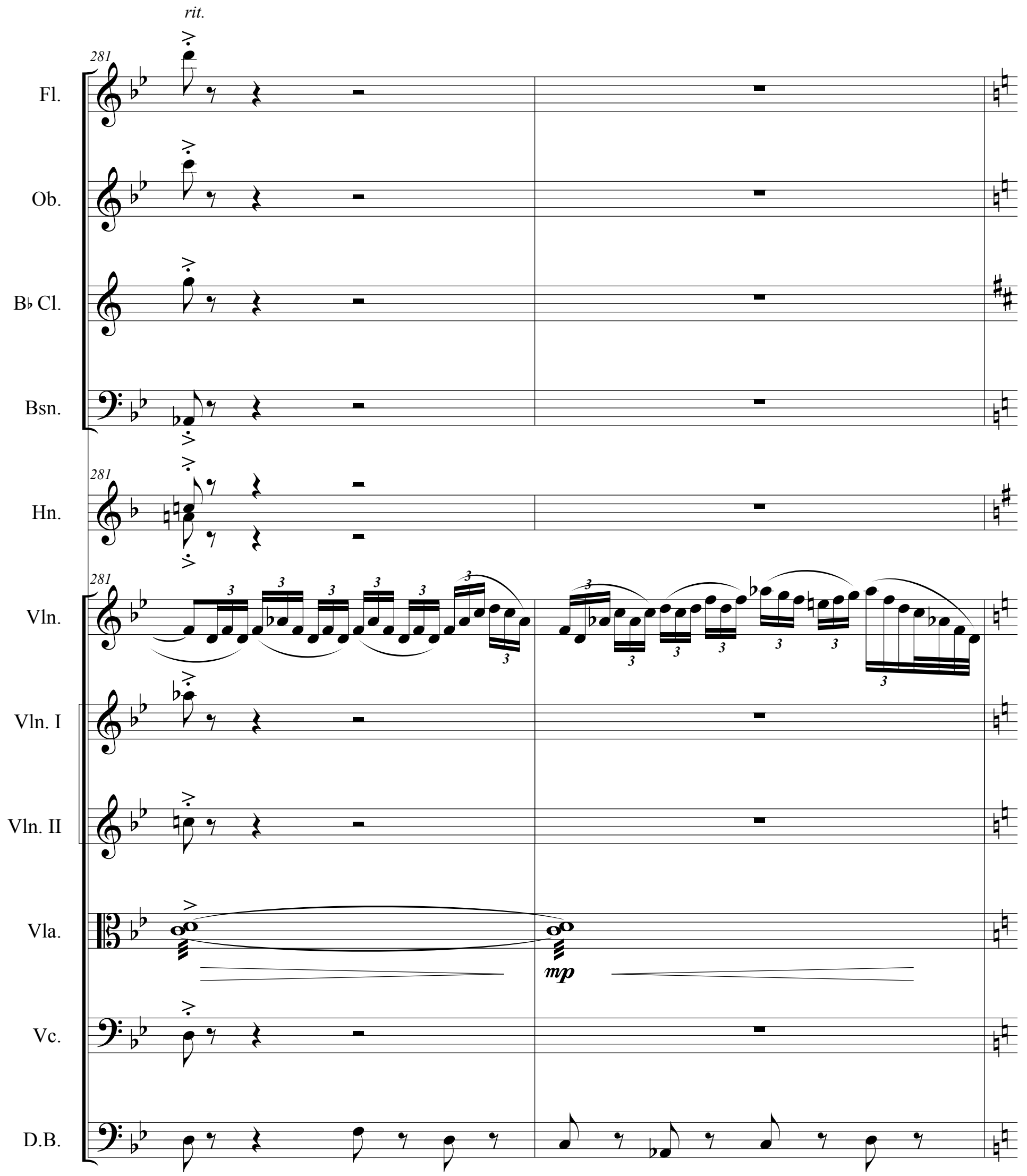


For Violin Solo and Chamber Orchestra

Score

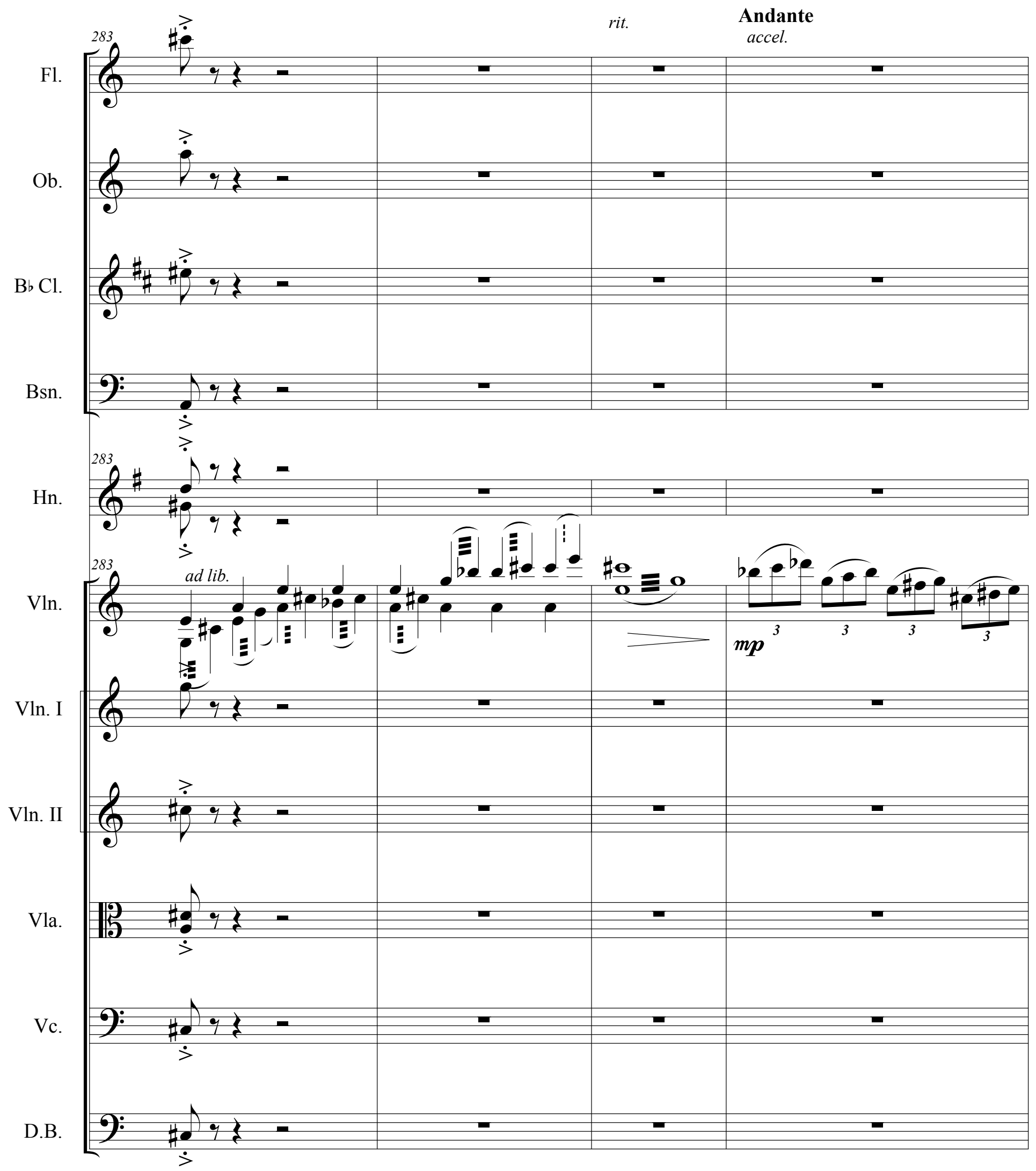


Score

For Violin Solo and Chamber Orchestra

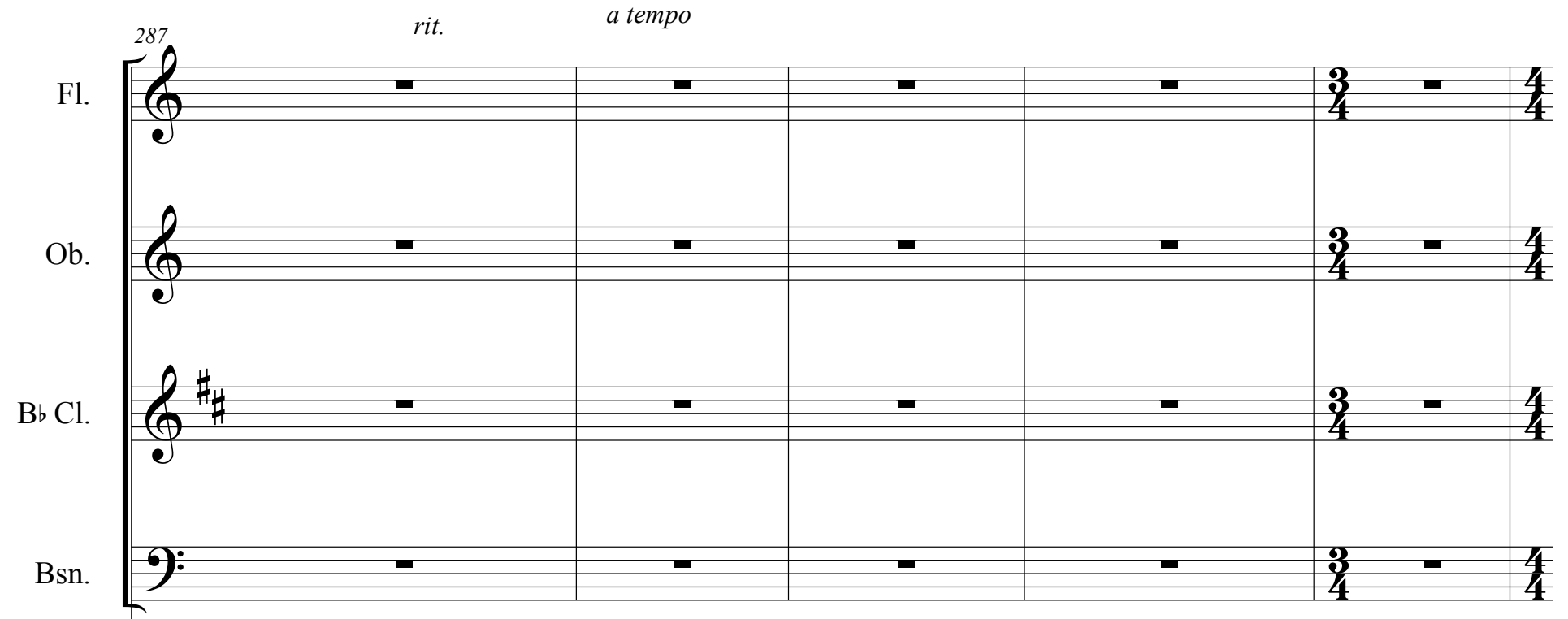

Hn.
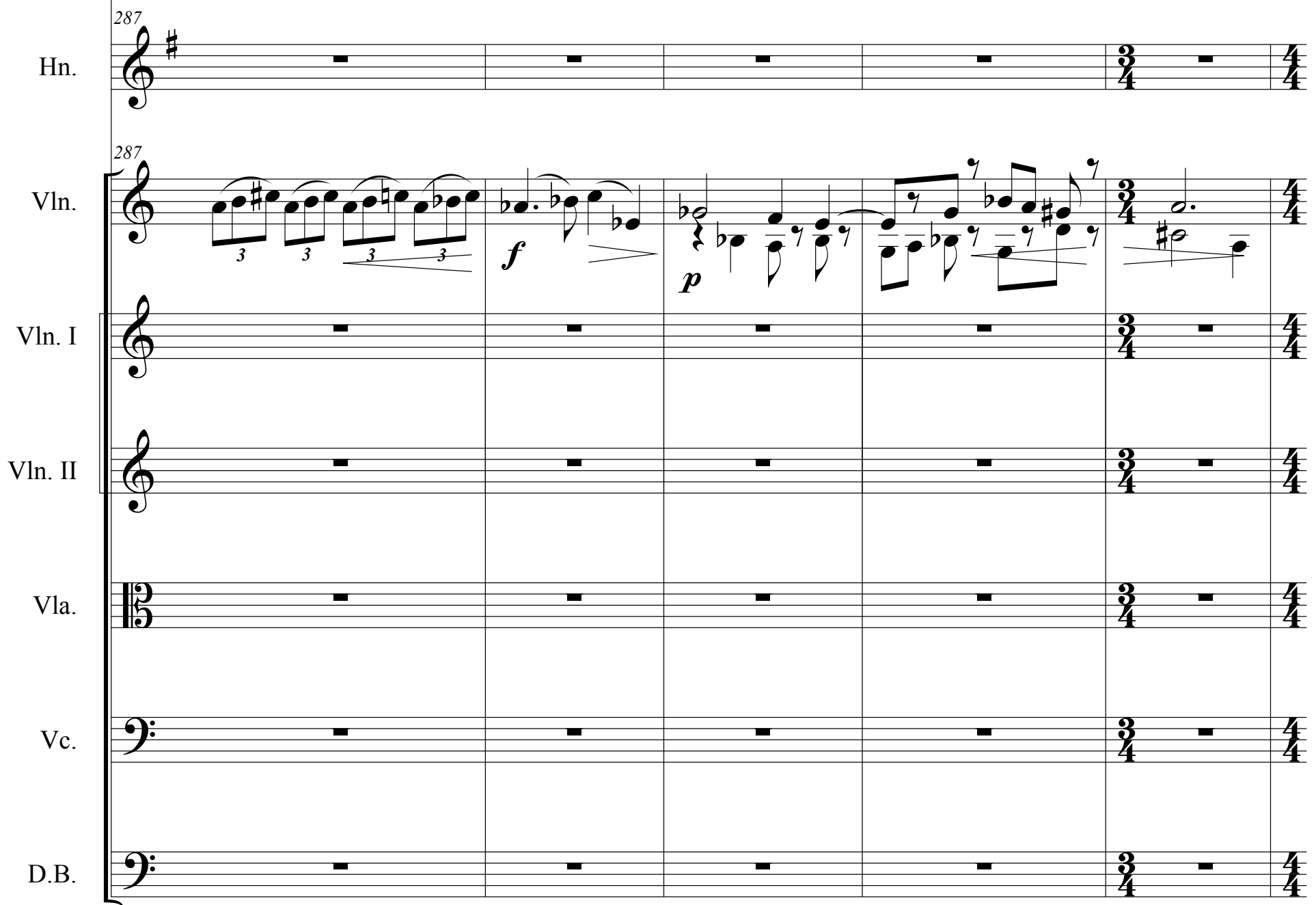


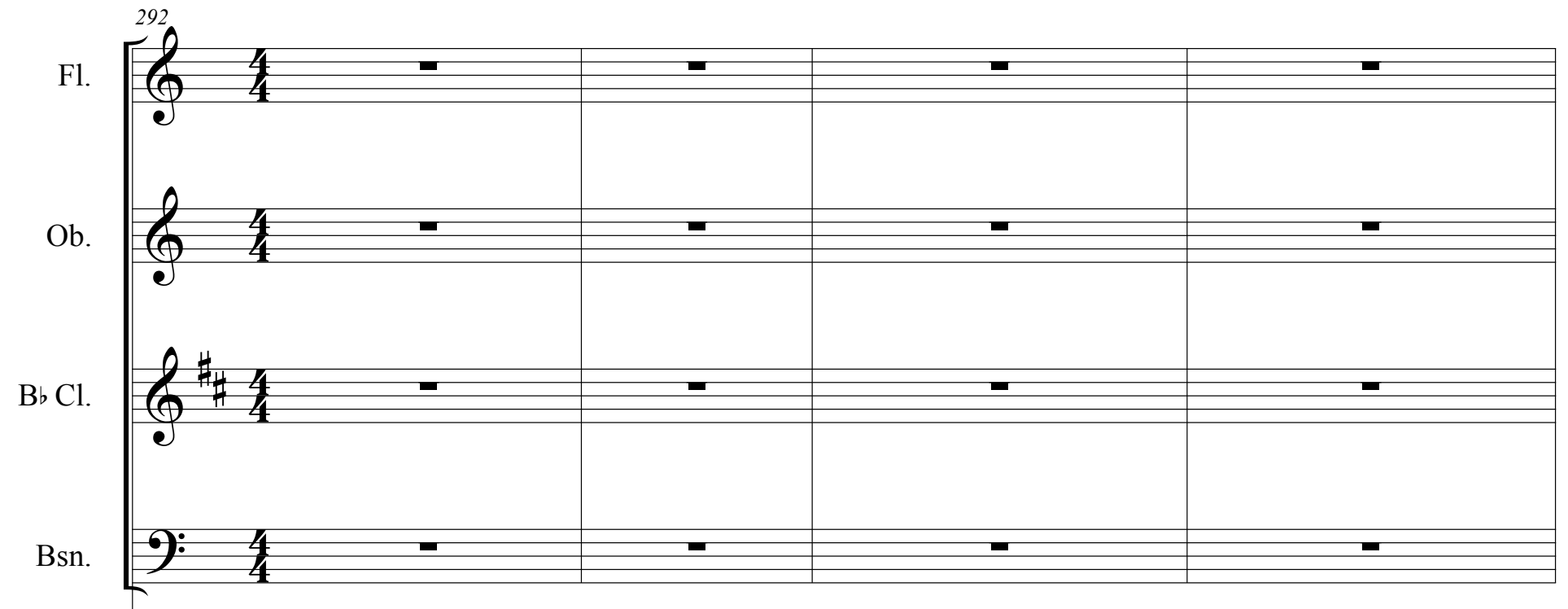

Hn.
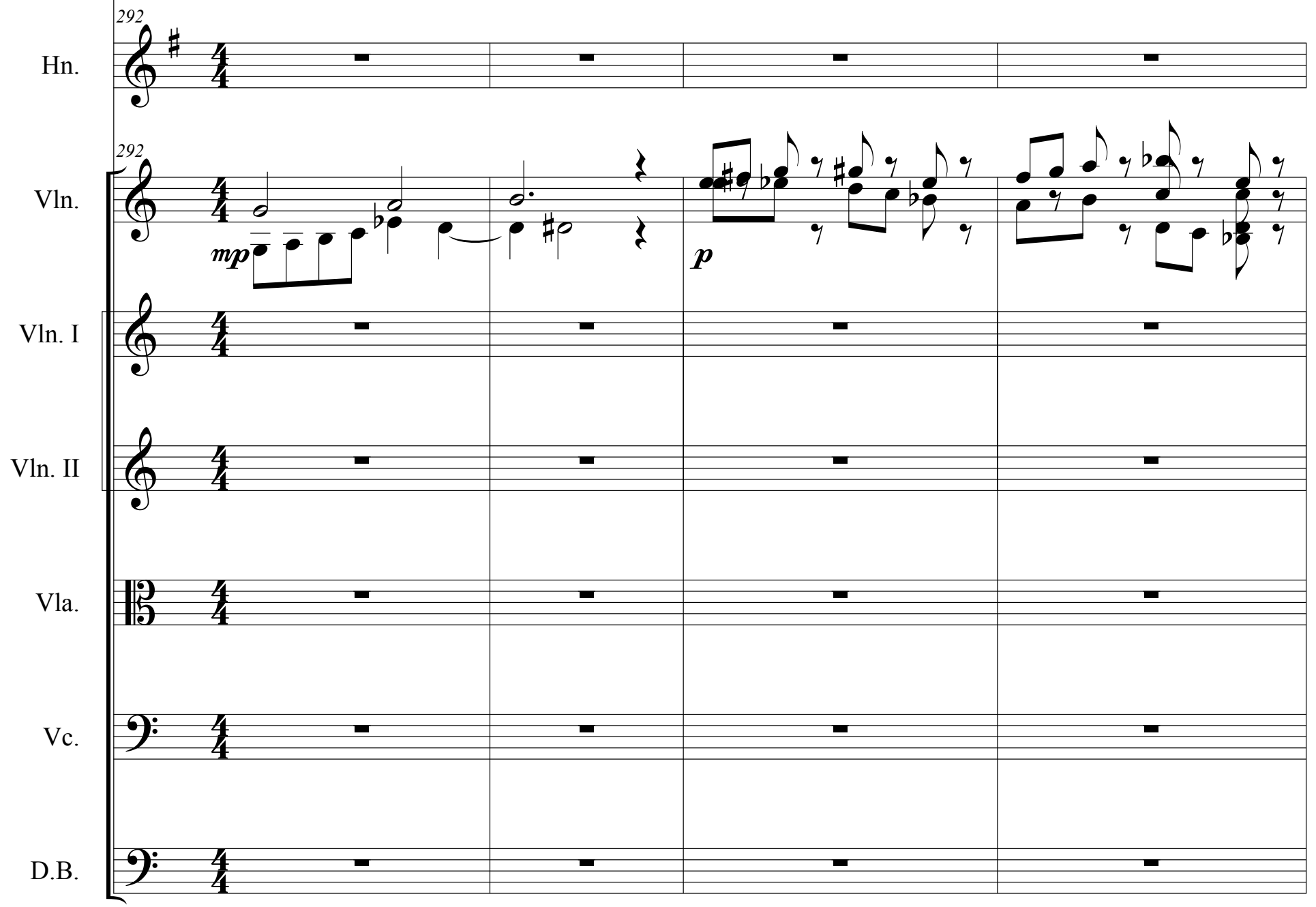
Score

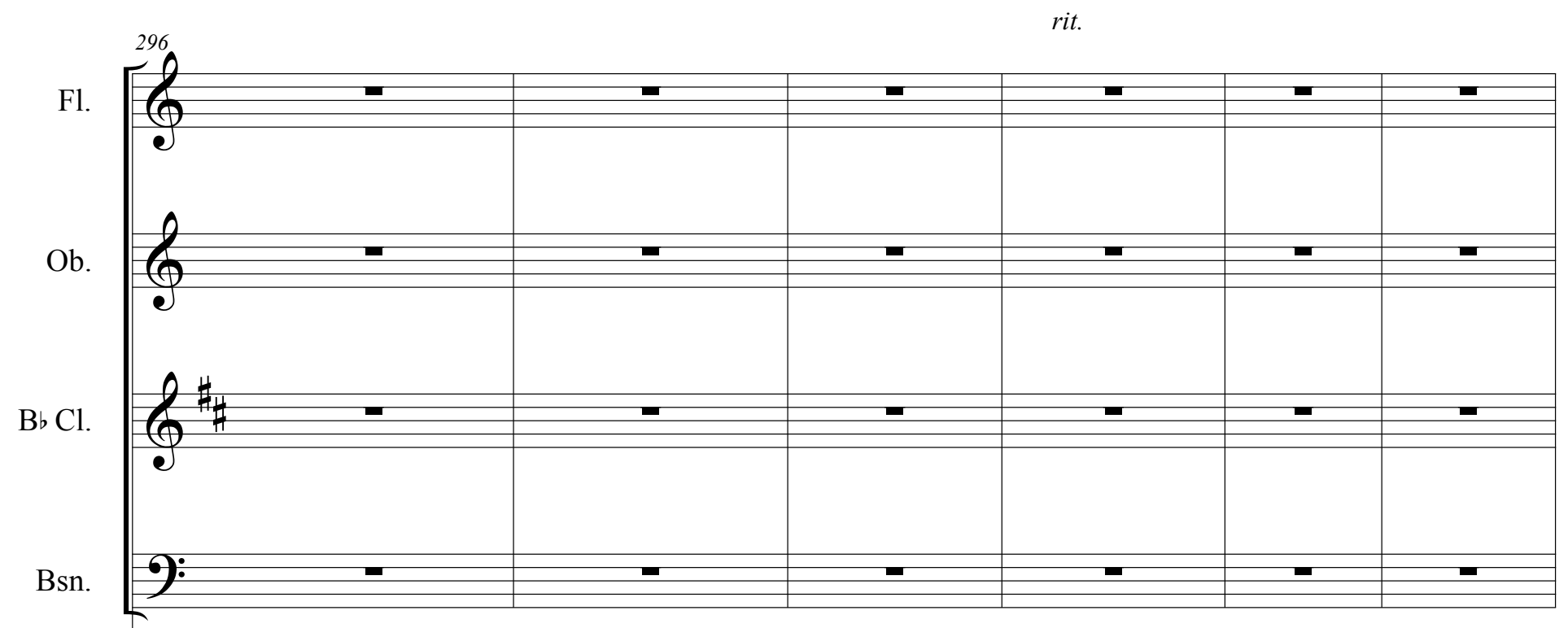

Hn.
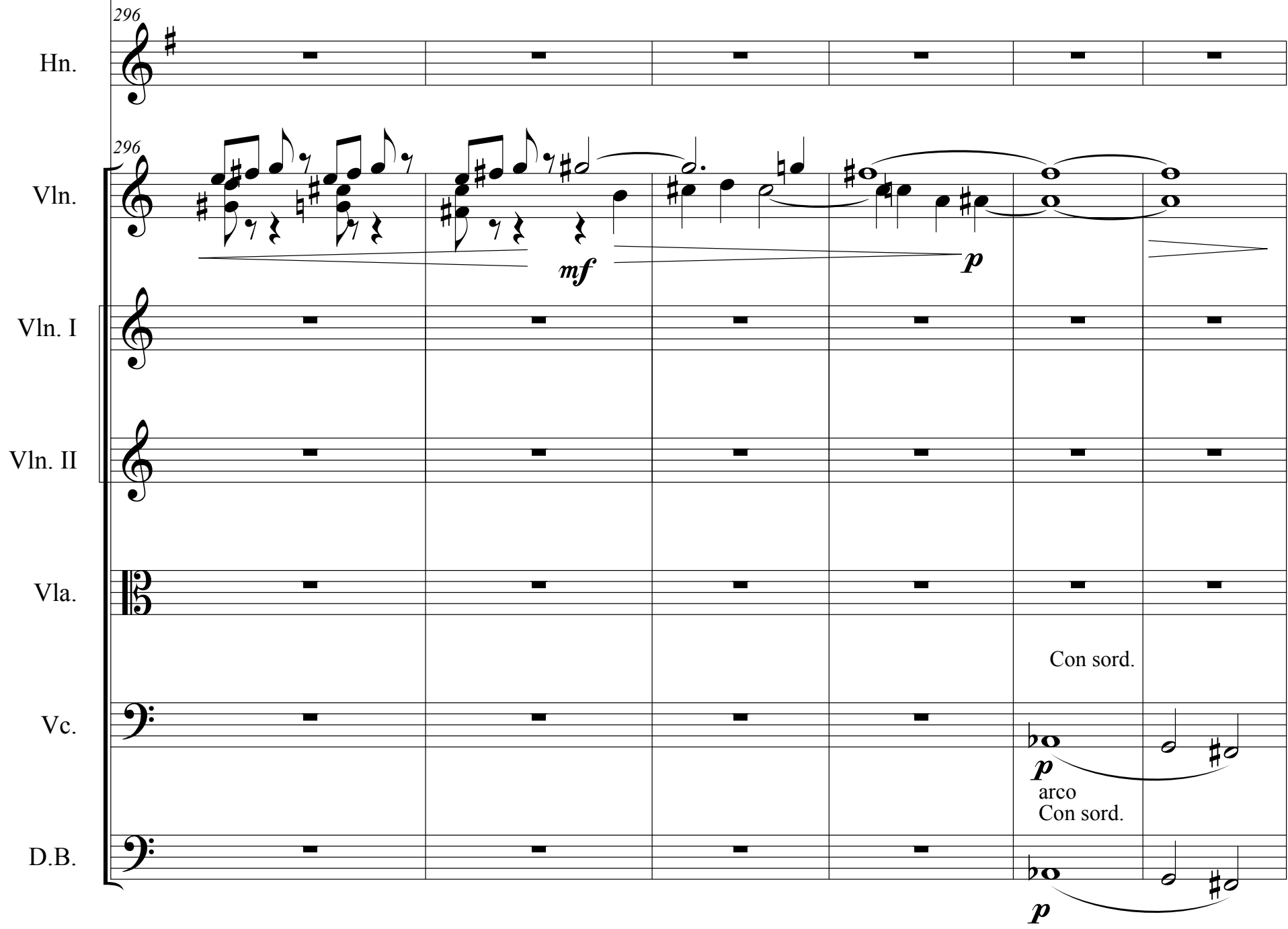


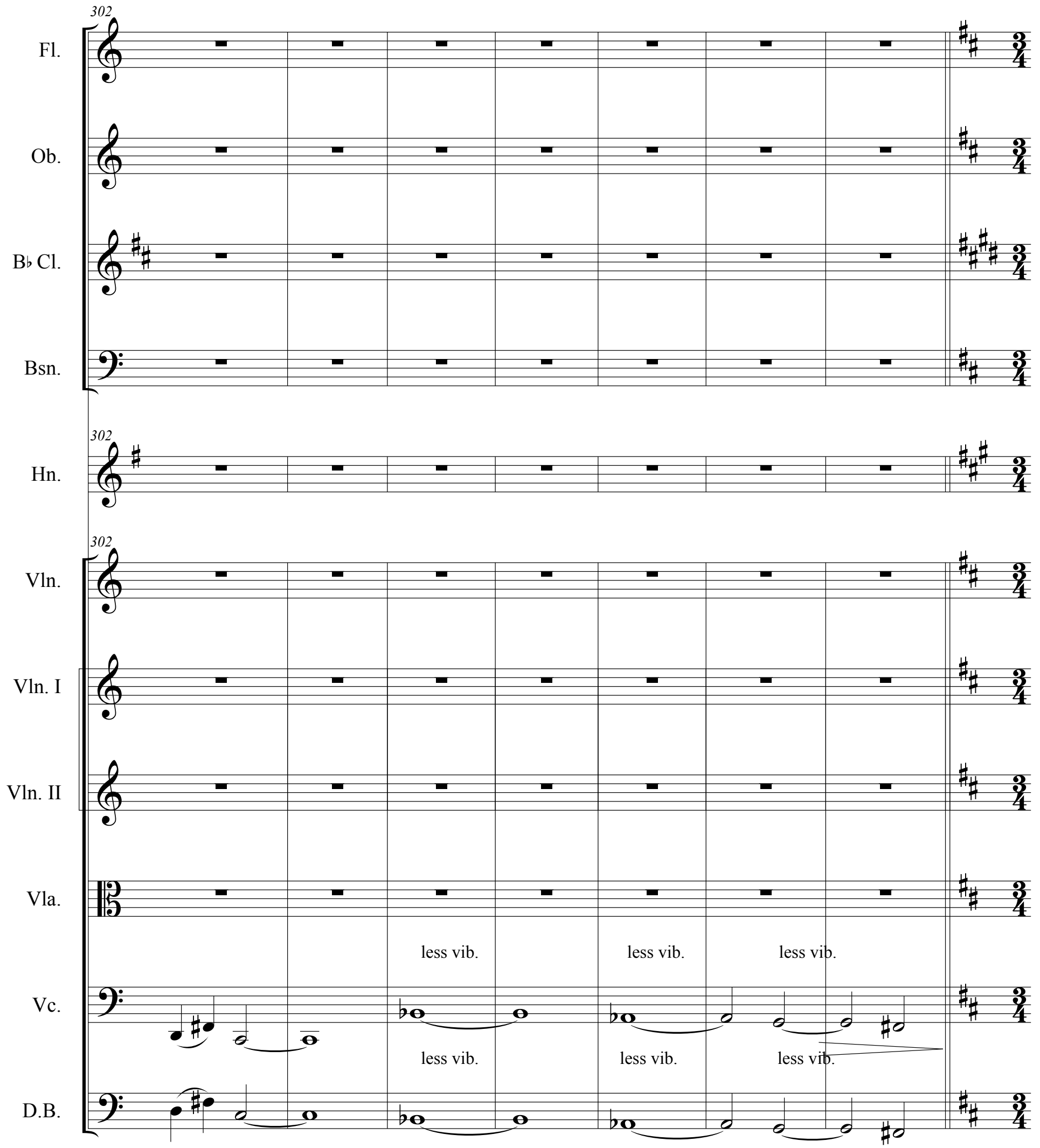


Score

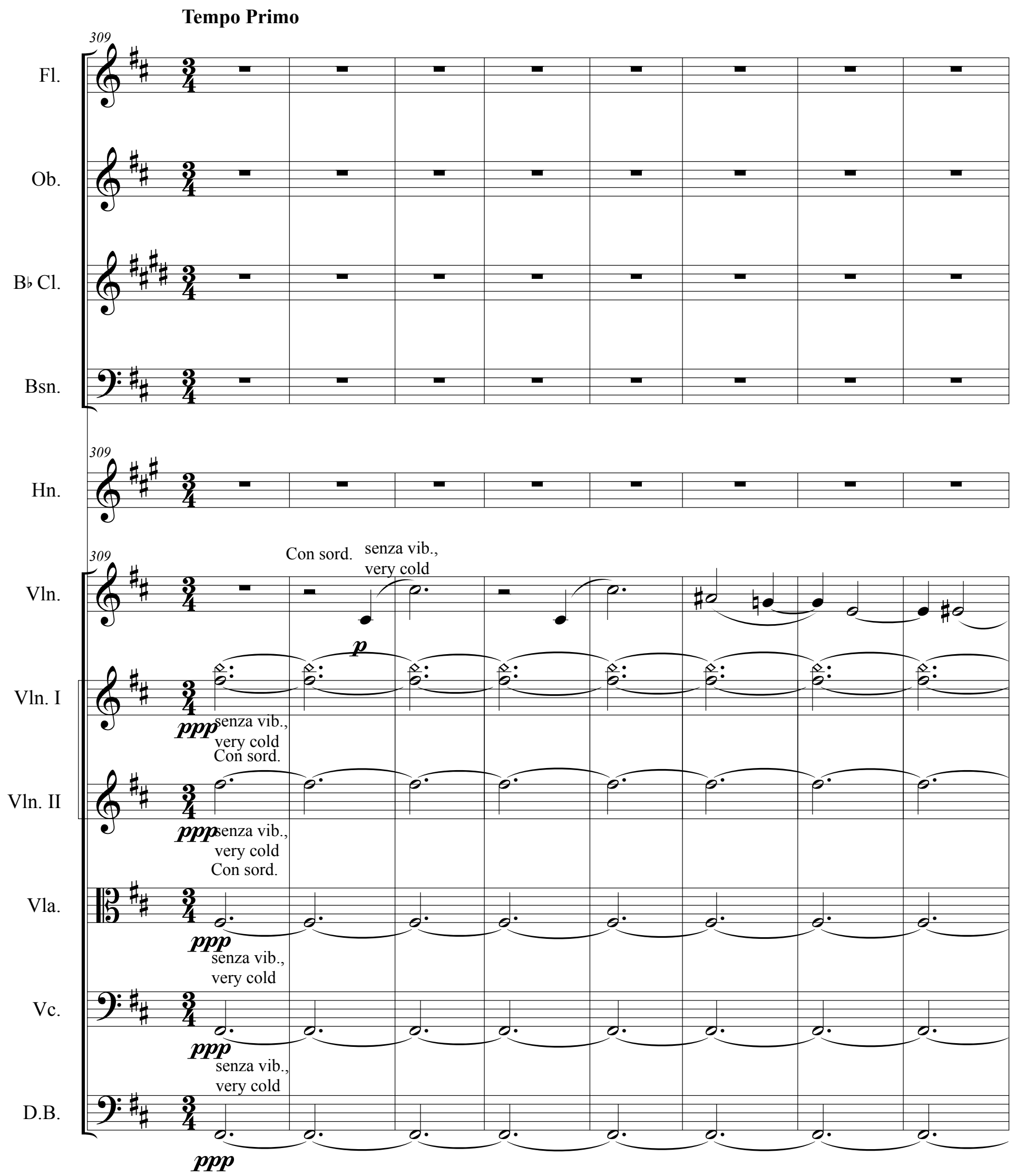



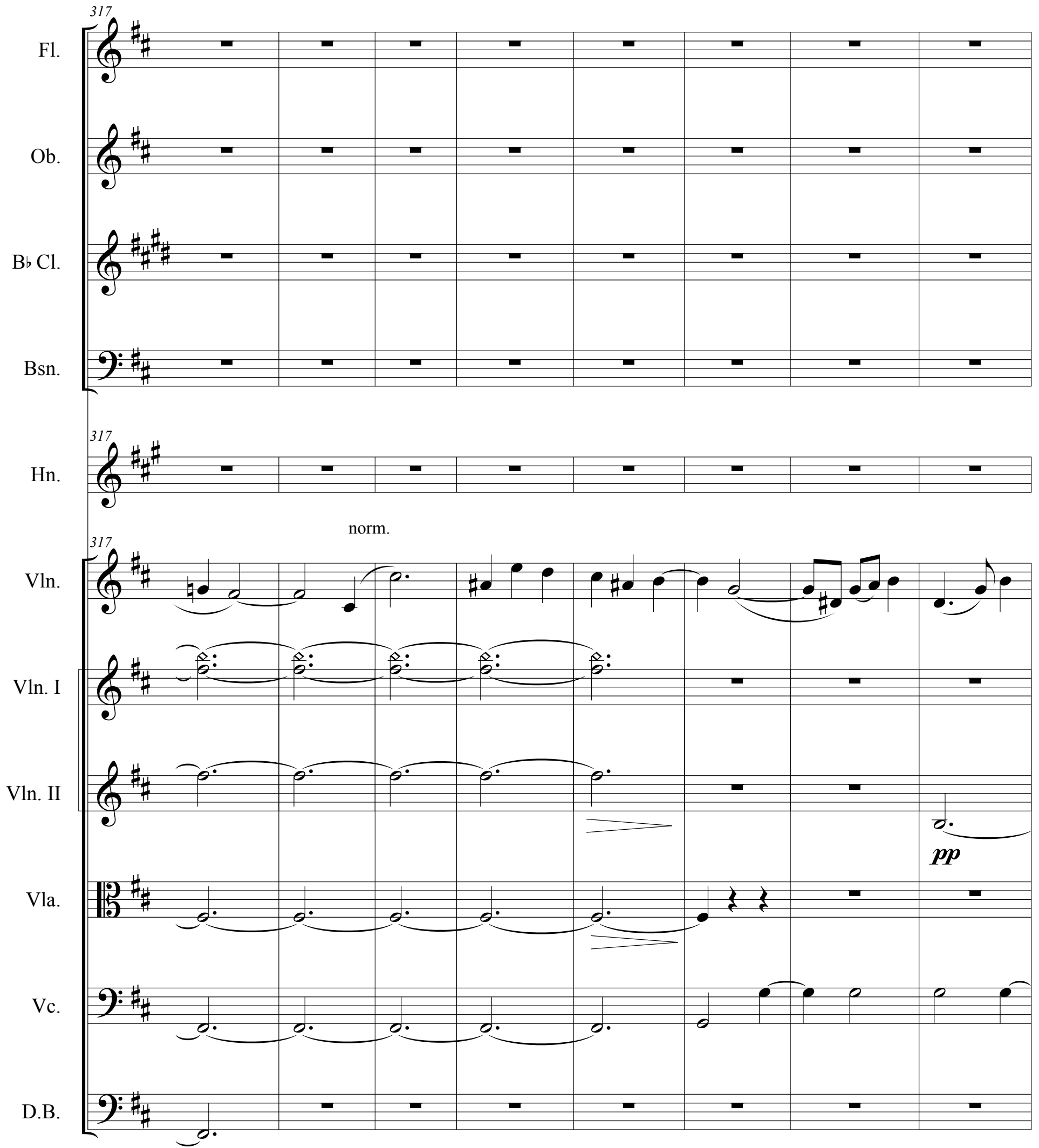
Score

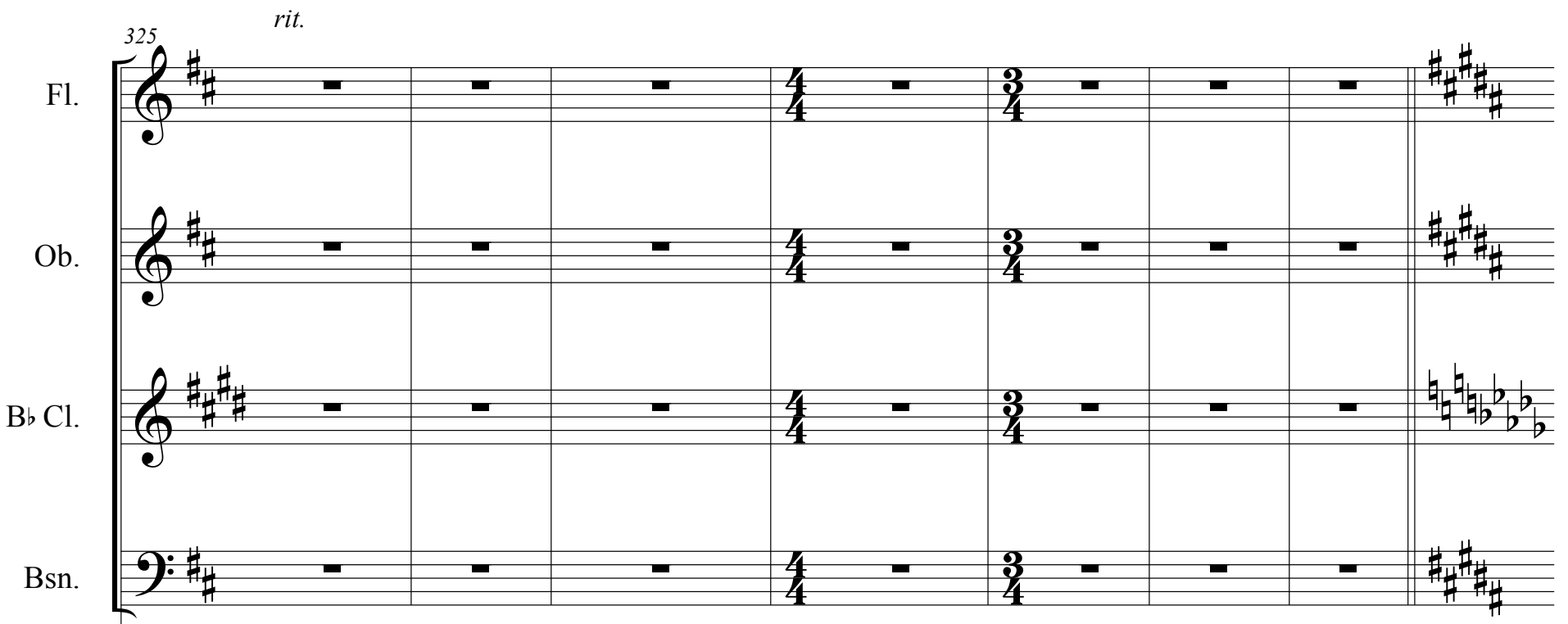

Hn.
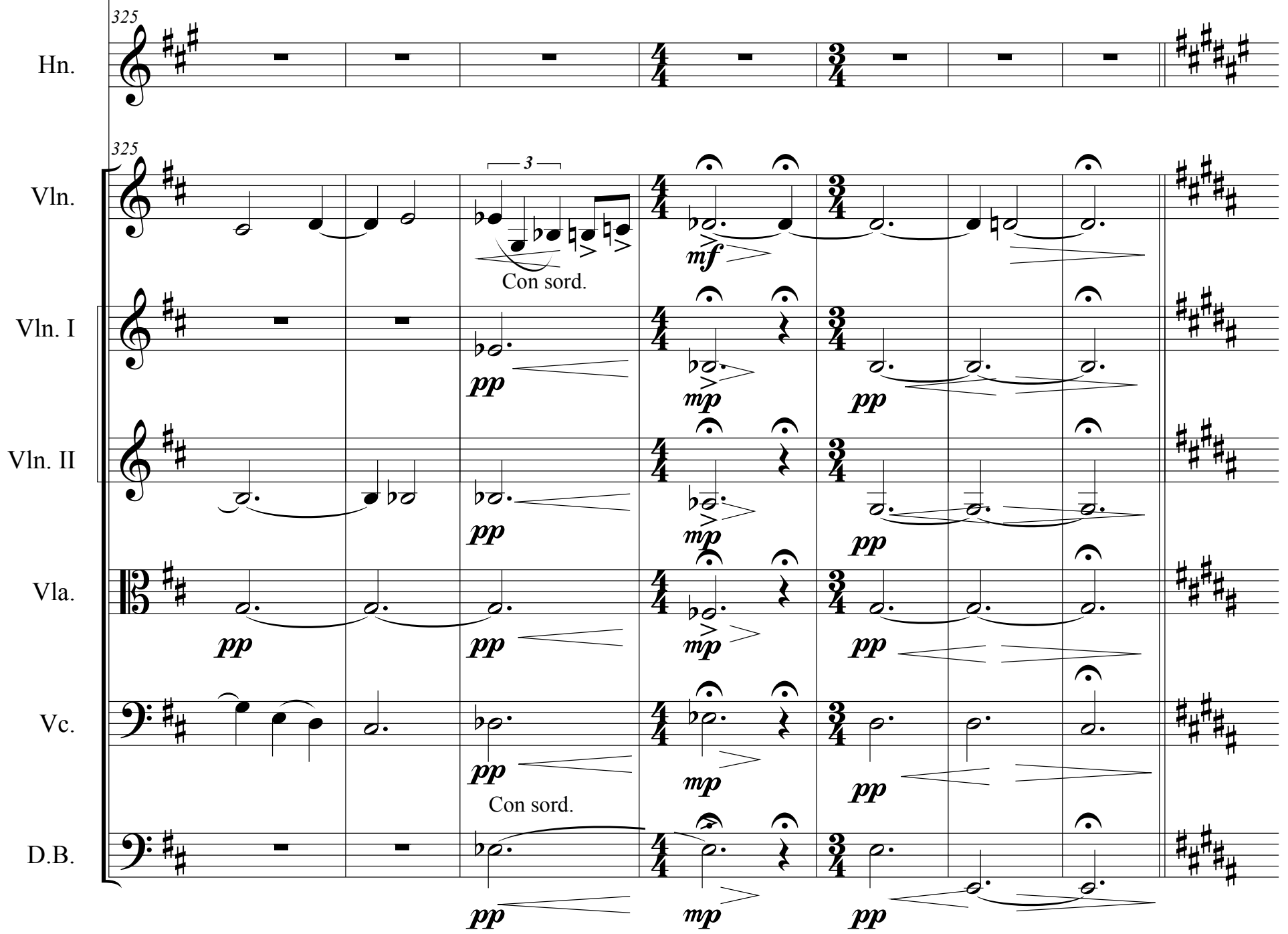
76

For Violin Solo and Chamber Orchestra

Quarter $=46$

Largo

Fl.

$\mathrm{Ob.}$

$\mathrm{Bb}$ Cl.

$\mathrm{Bb}$ Cl.

Hon.

$\int_{0}^{332}$

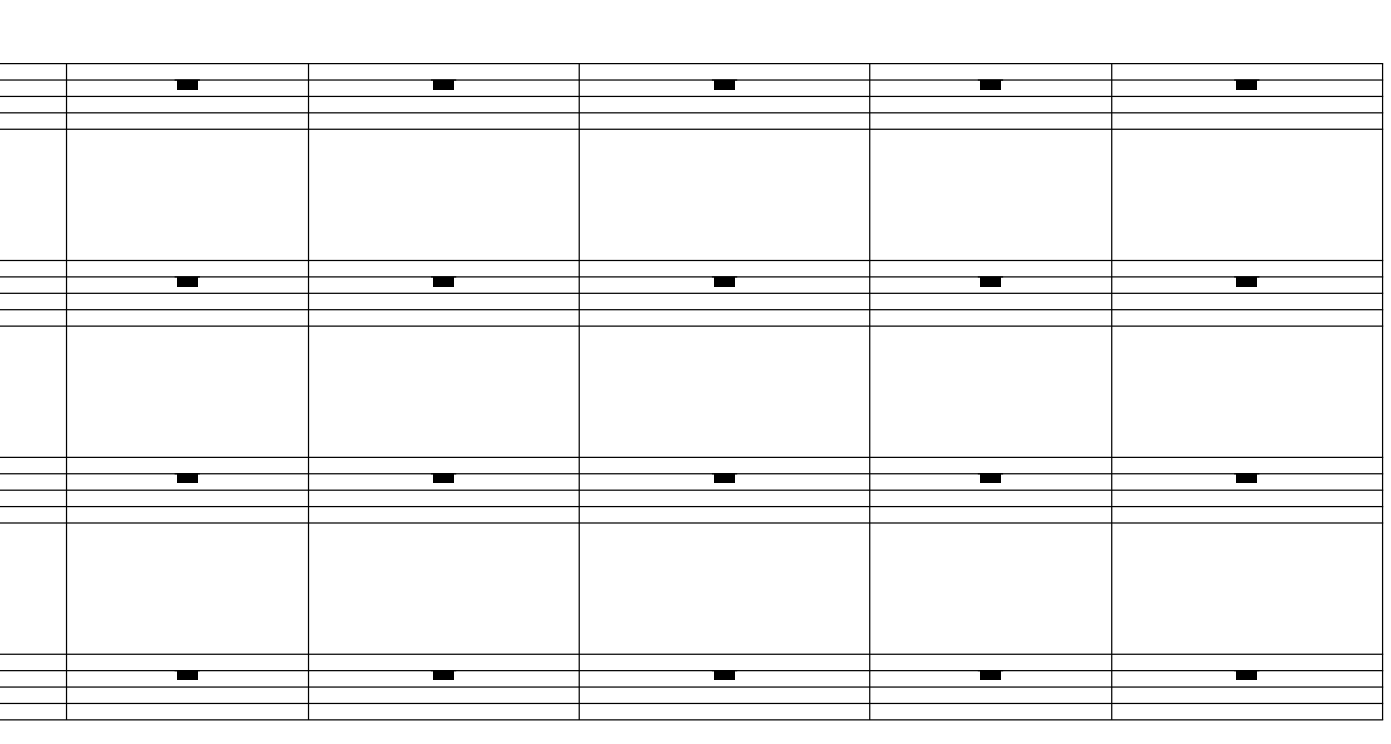

$\int_{0}^{332}$

V1n.

Vln. I

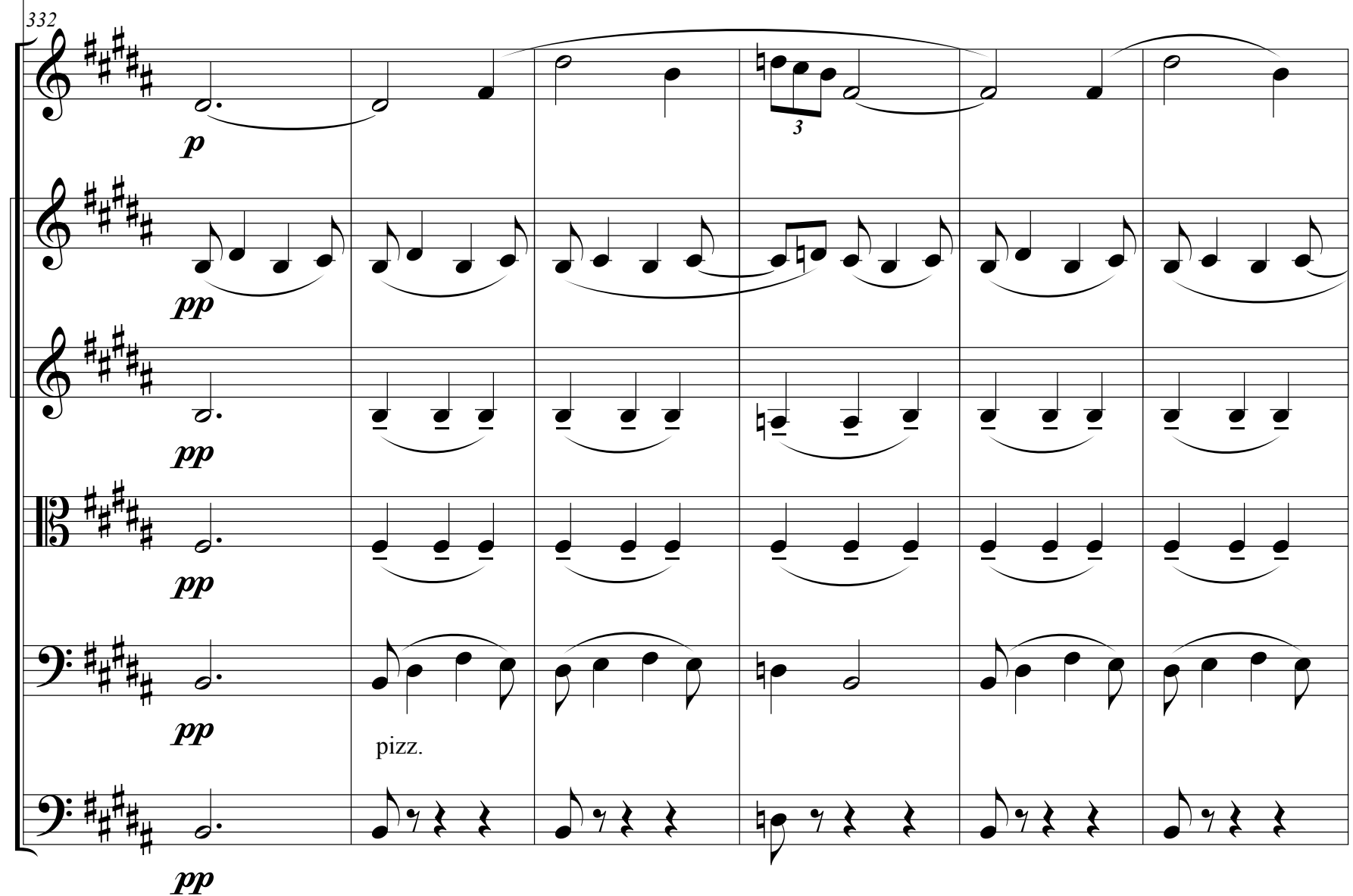

VIa.

Vc.

D.B.

$\boldsymbol{p p}$ 
Score

For Violin Solo and Chamber Orchestra

77

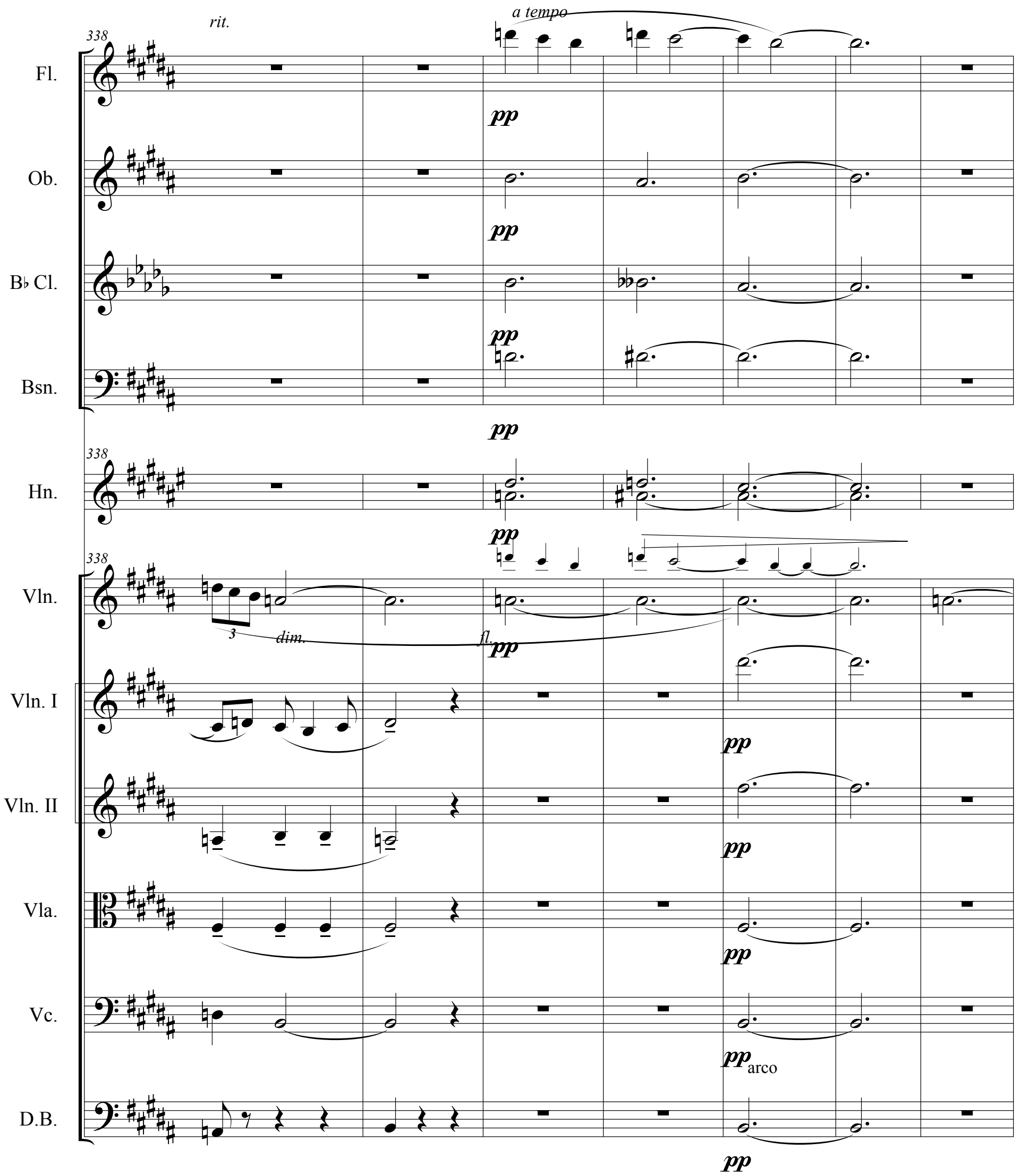


rubato

$\mathrm{Ob}$.

Fl.
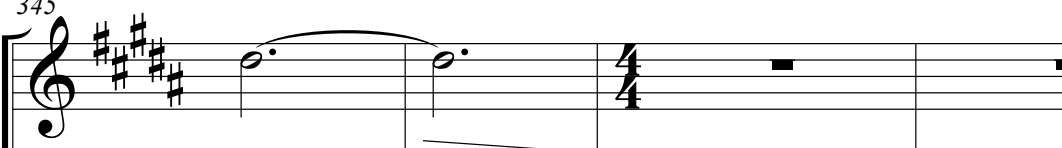

$\mathrm{B} b \mathrm{Cl}$.

Bsn.
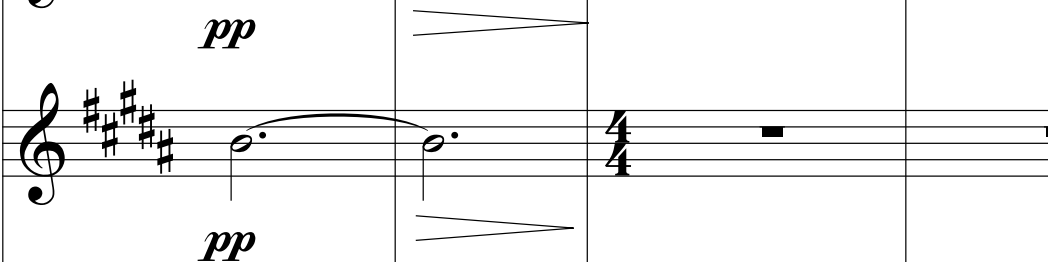

Hn. $\overbrace{0}^{345}$
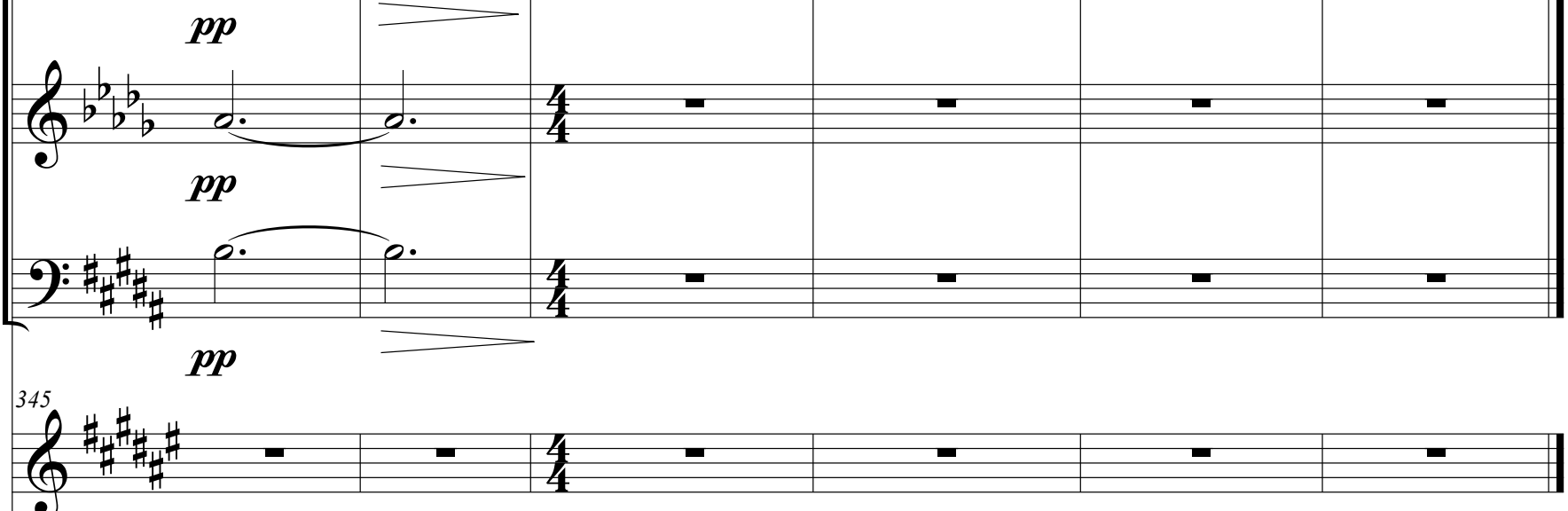

Vln. I

345

Vln.
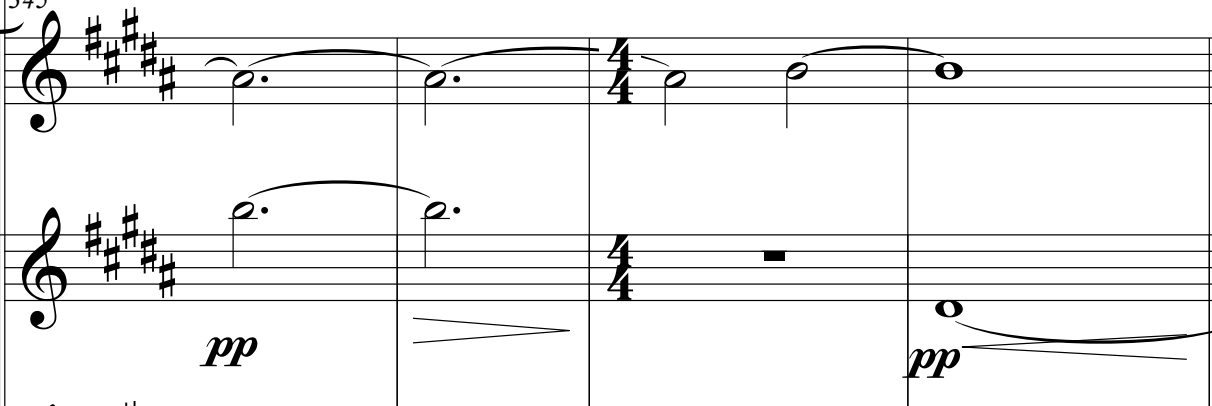

Vln. II
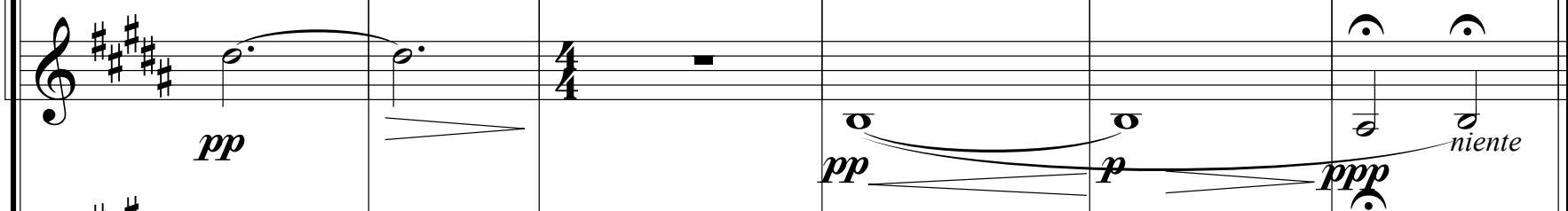

Vla.

Vc.

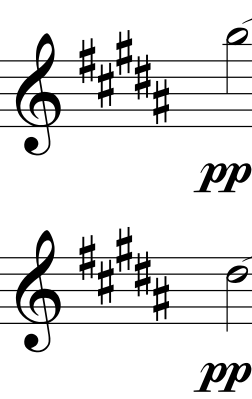

15 o.

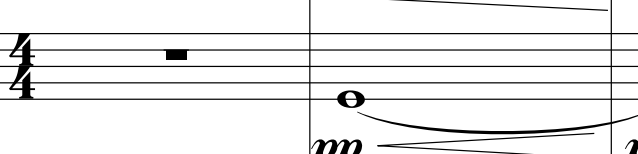

ก

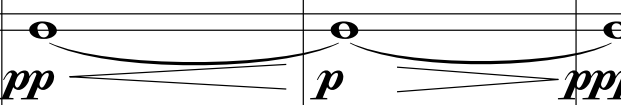

$p=0 p$

p niente

D.B.
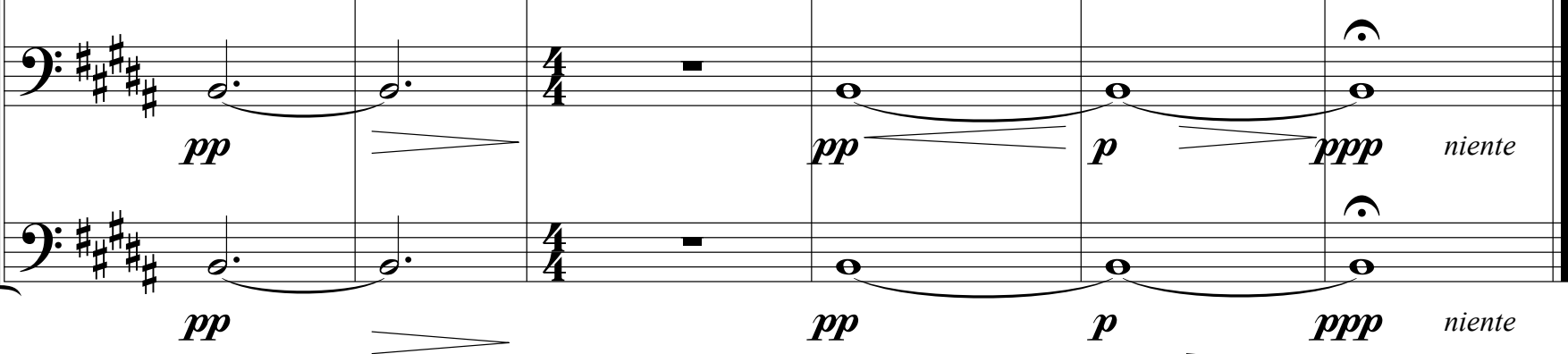